University of Arkansas, Fayetteville

ScholarWorks@UARK

Graduate Theses and Dissertations

$12-2013$

\title{
The Development of a Traumatic Brain Injury Bioreactor
}

Zachery Heller

University of Arkansas, Fayetteville

Follow this and additional works at: https://scholarworks.uark.edu/etd

Part of the Bioelectrical and Neuroengineering Commons, and the Computational Neuroscience Commons

Citation

Heller, Z. (2013). The Development of a Traumatic Brain Injury Bioreactor. Graduate Theses and Dissertations Retrieved from https://scholarworks.uark.edu/etd/960

This Thesis is brought to you for free and open access by ScholarWorks@UARK. It has been accepted for inclusion in Graduate Theses and Dissertations by an authorized administrator of ScholarWorks@UARK. For more information, please contact scholar@uark.edu. 
The Development of a Traumatic Brain Injury Bioreactor 
The Development of a Traumatic Brain Injury Bioreactor

A thesis submitted in partial fulfillment
of the requirements for the degree of
Master of Science in Biomedical Engineering

by

Zachery Edward Heller

Arkansas Tech University

Bachelor of Science in Mechanical Engineering, 2011

December 2013

University of Arkansas

This thesis is approved for recommendation to the Graduate Council.

Dr. Jeffery Wolchok

Thesis Director

Dr. Adam Huang

Committee Member

Dr. Kartik Balachandran

Committee Member 


\begin{abstract}
Approximately 1.7 million Americans experience a traumatic brain injury (TBI) each year. Concussive injuries are a subset of TBI in which blows to the head cause the brain to collide against the interior of the skull. Damage to the neurons, supporting cells, and surrounding extra cellular matrix resulting from these collisions can lead to permanent physical, cognitive, and psychological impairment. We believe the prevalence and clinical significance of concussive injures warrants research investment. To study brain injury following TBI, in vivo models have been the gold standard for TBI experiments. Although a valuable research alternative, animals are expensive, raise ethical concerns, and introduce experimental complexity than is necessary. In vitro systems provide greater insight and control into the fundamental cellular responses to injury despite sacrificing the complex and more realistic reactions. Following in the same desire to answer fundamental responses, our group has developed a bench-top device capable of delivering TBI mimetic impacts to cells in culture. Overall, the as built bioreactor can deliver impact decelerations of up to $300 \mathrm{G}$ in combination with strains up to $25 \%$ to as many as six cell inserts. The small footprint $\left(1^{\prime} \times 1^{\prime}\right)$ and inexpensive design $(\$ 1000)$ make it an ideal lab based system. While the initial testing was conducted on cells with a neuron phenotype we are primarily interested in using this system to explore the role of astrocytes in TBI. Specifically we plan to explore if changes to the typically neuro-supportive astrocytes following concussive impact may be in part responsible for the neuronal death observed following TBI, and in doing so potentially identify soluble markers of cell injury that could become part of a concussion diagnostic test.
\end{abstract}




\section{ACKNOWLEDGMENTS}

I would like to express my deep gratitude to my research advisor, Dr. Jeffery Wolchok, for giving me the opportunity to participate in his research endeavors. His tireless patience, enthusiastic guidance, and useful critique provided the essential feedback in becoming a better researcher, analyst, and presenter. I would like to thank Joseph Wyatt and Anna Arnaud, as well, for their help in collecting and analyzing impact durations and C8-DA1 attachment which provided a deeper characterization and validation for the bioreactor.

My grateful thanks also extend to my parents for their continuous support and relentless encouragement that furnished the required determination for my educational pursuits. 


\section{TABLE OF CONTENTS}

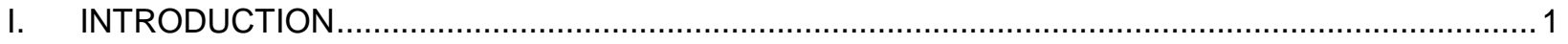

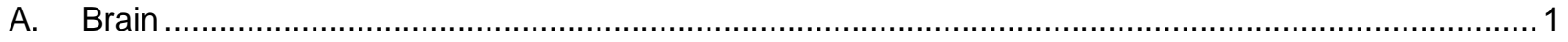

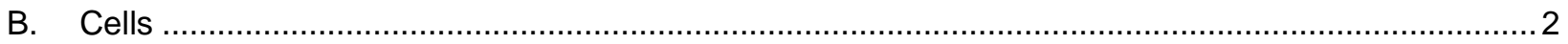

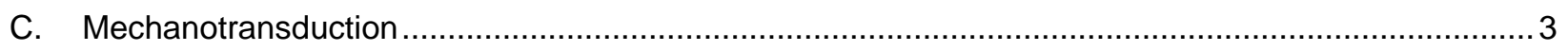

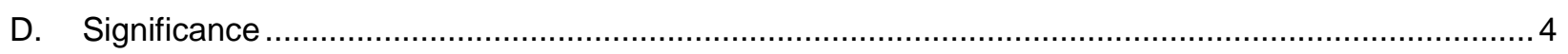

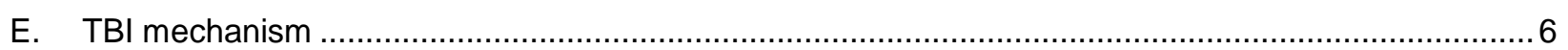

a. Jerk

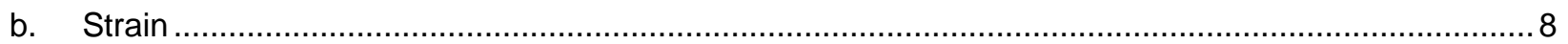

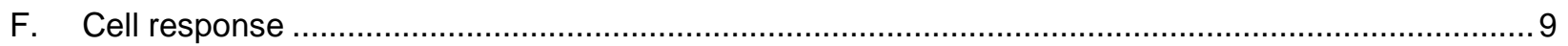

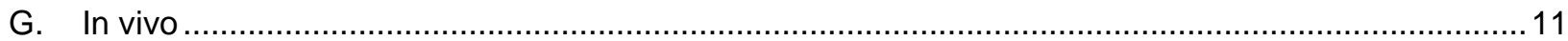

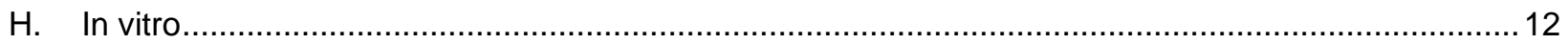

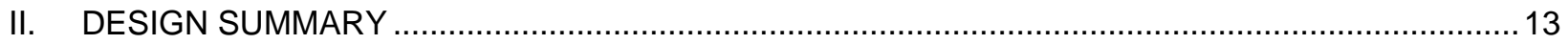

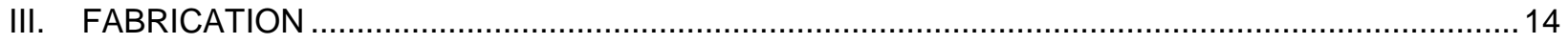

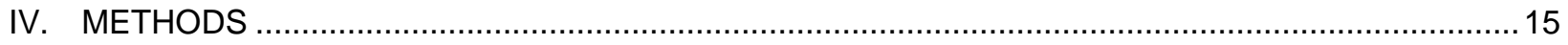

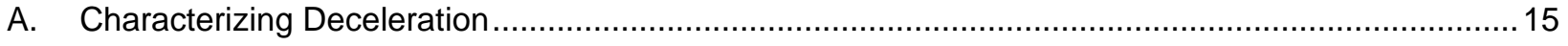

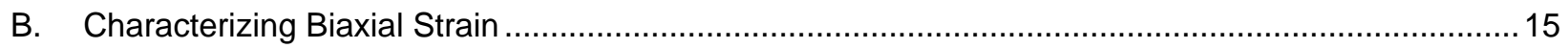

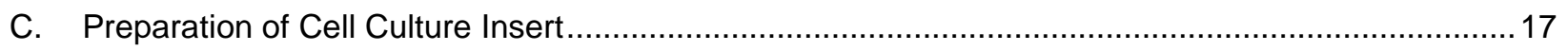

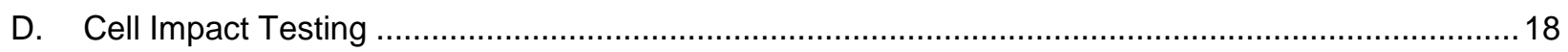

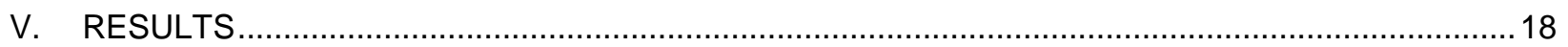

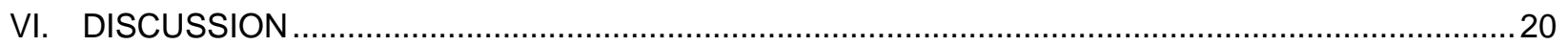

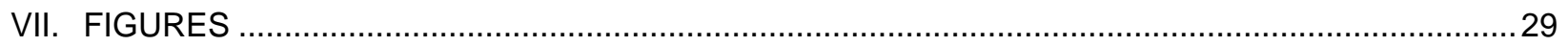

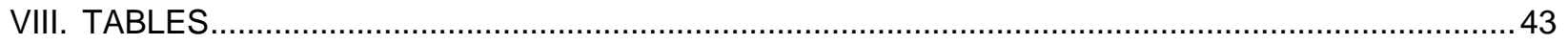

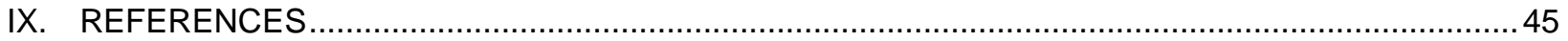

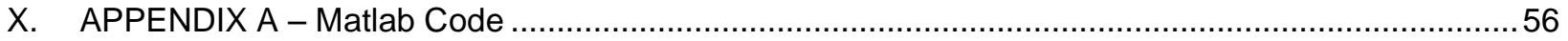

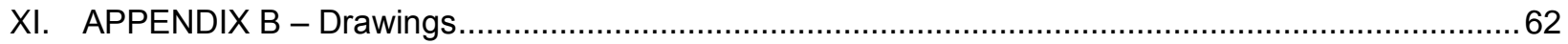

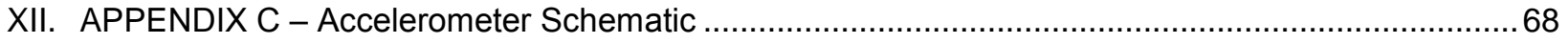




\section{INTRODUCTION}

\section{A. Brain}

"Men ought to know that from nothing else but the brain come joys, delights, laughter and sports, and sorrows, grief, despondency, and lamentations. And by this, in an especial manner, we acquire wisdom and knowledge, and see and hear, and know what are foul and what are fair, what are bad and what good, what are sweet and what unsavory; some we perceive by habit and some we perceive by utility... And by the same organ we become mad and delirious, and fears and terrors assail us, some by night and some by day, and dreams and untimely wanderings, and cares that are not suitable, and ignorance of present circumstances, desuetude and unskillfulness. All these things we endure from the brain."

Hippocrates: On the Sacred Disease, translated by Francis Adams

From all that we have discovered in the past century, it is surprising how resolute some early understandings still are. Responsible for discerning every signal from the sensory system, comprehending a response, and enacting a response by any mental and/or physical means; the central nervous system is considered one of the most complex networks in the world if not in the observable universe. For anatomical definition, this organ is parcellated into three main components: cerebrum, cerebellum, and brainstem. Although the relatively large cerebrum is divided into many regions, a general distinction in its histology can be observed. The outer region contains densely packed nerve and glial cell bodies, collectively called gray matter or the cerebral cortex. The inner region, called white matter, is predominantly made of nerve fibers and (axons). Roughly between 1300 to 1400 grams at maturity, the brain lies within the skull and is surrounded in cerebrospinal fluid which supports the brain structurally and functionally. The brain mass is majorly water (78\%) leaving the numerous compositions of lipids (9-17\%) and proteins $(8-12 \%)$ responsible for the brain's functionality ${ }^{89}$. A wide range of values exist for the brain's mechanical properties, but one thing is certain: it is one of the softest tissues in the body. Recent studies of porcine tissue properties have observed that the brain softens as it deforms ${ }^{29,115}$. Described as a viscoelastic material, the rat and human brain have an elastic modulus approximately between 0.1 to $16 \mathrm{kPa}^{42,51,71,95}$. For a frame of reference; bone, artery, and soft-muscle elastic moduli are $15-30 \mathrm{GPa}$, 0.1-1 MPa, and 10-100kPa; respectively ${ }^{94}$. These studies characterizing the macroscopic physical characteristics of the brain derive the elastic modulus from the measured shear modulus. What makes these characterizations so difficult is not only the quality of the sample, but that stiffness varies in 
individuals ${ }^{71,91,141}$ old and young ${ }^{123}$, healthy and sick $^{101,140}$. Regardless of this difficulty, the main concept is that the brain is very susceptible to mechanical forces.

\section{B. Cells}

When considering the cells operating the many intricate networks, the cell types are neurons, glial cells, and microglial. Neurons are the basic functional unit of the central nervous system (CNS) and relay electrical signals in the motor and sensory systems. The neuronal cell membrane passes an electrical signal along its axonal process. This cellular characteristic is called excitability and is dependent upon initiation of a cell membrane's action potential. The action potential is the elevated potential difference that exists across the neuronal cell membrane. By manipulating specific ionic channels in the membrane, the potential difference can reach a threshold that subsequently triggers the surrounding membrane's potentials to reach their action potential. This propagation of this electrical signal allows the neuron to transmit along its axon. Not only capable of electrically signaling, neural structures called chemical synapses can pass chemical signals to other neurons via neurotransmitters. Released neurotransmitters bind to receptors on the postsynaptic cell and can initiate any number or variations of a response. These signals are not based on an "all-or-nothing" process from the action potential. To facilitate neuronal functions like synapse signaling, neurons have glial networks surrounding them. From enveloping and protecting a neuron's myelin sheath to responding to injury and maintaining the neuron's homeostasis, roles of glial cells are seemingly as numerous as their numbers ${ }^{129}$. Astrocytes are present contiguously and non-overlapping throughout the entire CNS. With no region devoid, astrocytes outnumber neurons 5 to $1^{129}$. Dependent on the tissue region, they appear having several stem branches with fine processes or having long fiber-like processes. Although expressing potassium and sodium channels, astrocytes do not propagate action potentials along their processes like neurons but use chemical transmitters and mediators to signal. Astrocytes not only play a role in synapse function but also in development, blood flow regulation, fluid, ion, $\mathrm{pH}$, and transmitter homeostasis, CNS metabolism, and the blood brain barrier. Recent studies show certain region-specific astrocytes even control breathing ${ }^{54}$. But one of its more important roles is in CNS injury and healing. Called reactive astrogliosis, astrocytes undergo several cellular and functional changes to heal and contain the damaged area. Astrogliosis is not an "all or nothing" process but a "finely gradated continuum of progressive changes in gene expression and cellular 
changes $^{129}$." Gliosis refers to the glial scarring that develops during the healing process but this seemingly-specific term encompasses many other diverse functions of activated astrocytes. One recent study reported astrocytes clearing apoptotic cells by engulfment so their cytotoxic effects would be contained $^{83}$. This task was originally thought to be strictly designated to the microglial.

Microglia have reparative functions, as well as being part of the brain's immune defense. similar to macrophages, they phagocytize foreign or harmful products and are extremely sensitive to any inflammatory or stress stimuli. Microglia also regulate T-cell responses through the presentation of antigens. Without an adaptive immune response, the range and speed of microglial reaction is pivotal and necessary for preventing further damage ${ }^{1}$. The lack of antibodies within the brain is due to one of the main parenchyma tissues: the blood brain barrier. This tissue is comprised of polarized endothelial cells responsible for the control and restriction of substance movement. Separating the general body's blood circulation from the brain's extracellular cerebral spinal fluid, the blood brain barrier contributes to the homeostasis of the brain. While water and specific electrolytes readily cross the membrane barrier, specific transport processes control the passing of glucose and some amino acids. This tissue bars not only deleterious substances from affecting the brain but also blocks beneficial ones like antibodies. The latter is a likely reason for the highly-reactive nature of the brain's immune system. To structurally support and position all the cell types and their variations, the extracellular matrix (ECM) is uniquely composed. Common matrix proteins like collagens, fibronectin, and laminin are nearly nonexistent in the brain. The majority of the brain's ECM is fabricated of lecticans, tenascins, and hyaluronic acid ${ }^{122}$. These ECM molecules are suspected of having trophic effects on neuronal cells and neurite outgrowth and may even help in the resistance of tumor invasions from non-neuronal origins. Not classically thought for its mechanical interactions, parenchyma cells of the brain have many functions that require or generate a mechanical response.

\section{Mechanotransduction}

Many normal cell functions like gene expression, cell adhesion, and fluid homeostasis are influenced by mechanical forces throughout the body. So it is no surprise that these same cellular components are found in the brain. The plasma membrane responds to force as nonlinear functions of 
strain which defines it as a viscoelastic or non-Newtonian fluid ${ }^{32}$. Any membrane deformation can affect the activity of ion channels at the millisecond-timescale. Mechanosensitive ionic channels can be activated by tension and stress in the plasma membrane, and various types regulating potassium, sodium, calcium, and chloride ions are present in the $\mathrm{CNS}^{2,60}$. These channels are capable of affecting neural activity but how forces affect these channels and cellular function is less known ${ }^{134}$. Within the cell, we know actin allows dynamic structural plasticity and a three-dimensional array of force transducers for the cytoskeleton ${ }^{48,72}$. In regards to neurons, regulation of axonal growth-cone dynamics is determined by actin-generated forces. Microtubules contribute to regulation of dendritic spine morphology and synaptic plasticity ${ }^{66,68}$. Abundantly located in myelinated axons, neurofilaments can modulate mechanical forces in axons $^{78}$. Due to their structure, the side-arms of the neurofilament's long filamentous fiber are believed to resist compression loads ${ }^{21}$. Integrins have other roles for CNS cells than link ECM proteins to the cytoskeleton. Neuronal integrins regulate the formation and maturation of synapse, and play a supporting role in ECM signals that influence synaptic homeostasis and plasticity ${ }^{27,36}$.

The micromechanical energy within the brain is commonly present and crucial for normal function, but understanding the threshold when cellular-mechanical features fail is pivotal to understanding the deleterious consequences of TBI. As the result of mechanical activation of tetrodotoxin-sensitive sodium channels; diffuse axonal injury (DAI) can result in neurodegeneration from excitotoxity ${ }^{139}$. Extracellular proteolysis of the ECM is known to be initiated by plasminogen activators and matrix metalloproteinases in response to brain trauma ${ }^{80}$. This negative remodeling leads to the known cell death that occurs during the healing process, but the underlying instigation of this response is unknown ${ }^{80}$. Understanding these mechanical stimuli for injury is needed to treat the silent epidemic of traumatic brain injury (TBI).

\section{Significance}

Out of the 1.7 million occurrences of TBI in the United States, almost half a million will be annual emergency visits involving children; in fact, most likely to suffer a TBI are children newborn to 4 years old, teenagers $14-19$, and the elderly 65 and older ${ }^{31}$. Surprisingly, the majority of these cases are the result of falls (35\%) followed by motor-vehicle accidents. $75 \%$ of all cases are diagnosed as mild; however, TBI is 
a contributing factor to almost a third of all deaths in the US, and 52,000 Americans die each year from a TBI-related event. As far as neurological diseases, it is more common than epilepsy, multiple sclerosis, spinal cord injury, and amyotrophic lateral sclerosis, combined ${ }^{63}$. How close these estimates of TBI cases are to their true numbers cannot be determined; as many are believed to go unreported. From the known cases, the resulting economic burden of TBI from direct medical costs and indirect costs is approximately $\$ 60$ billion annually ${ }^{45}$; in comparison, Americans spend $\$ 4.5$ billion each year at McDonald's. The seriousness of the damage wrought by TBI often makes it known as a "silent epidemic" because TBI is not fully understood. Not only is it difficult to determine what to look for but also how to do so. The organ's delicacy and importance leaves an invasive observation undesired and pushes for other alternatives.

Current diagnosis of TBI is based on medical history, neurological examination results, clinical assessment scales, and neuroimaging. A popular assessment scale for emergency first responders, the Glasgow Coma Scale is a descending numerical scale from 1 to 15 . The scale is based on the efficiency of the patient's response of verbal, motor, and eye-movement. This test is dependent on the patient response which may be compromised from accurately conveying the unseen damage. Loss of consciousness used to be a symptom heavily relied on for brain damage and function, despite the fact that the specific mechanism is not clearly understood ${ }^{126}$. Reporting only if loss of consciousness was a post-injury symptom, an older report determined the number of sports-related TBI's occurring in the U.S. at 300,000 per year ${ }^{132}$. Newer studies investigating on-field symptoms of concussed athletes noted that only $8 \%{ }^{125}$ to $19 \%{ }^{30}$ of known TBI cases had loss-of-consciousness as a symptom suggesting sportsrelated TBI's to be as high as 3.8 million $^{75}$ each year. Neuroimaging such as computed tomography (CT) and magnetic resonance imaging (MRI) offer a supplementary method to determine intracranial abnormalities, like hemorrhages, but lower levels of TBI may not have structural changes; $3-10 \%$ of mildTBI cases have hemorrhages ${ }^{9}$. Most symptomatic mild-TBI patients have normal CT and conventional MRI scans ${ }^{11}$. These quasi-diagnostic tools only provide qualitative measurements of what the injury could be.

Properly identifying and diagnosing milder forms of TBI becomes exceptionally crucial when a secondary insult is possible. Although many deleterious results arise from the initial injury, potentially 
severe consequences manifest after a repeated insult relatively close in time to the original. Current data from several animal studies support the idea that biochemical and physiologic changes can be exacerbated by mild insults repeated within a specific time window ${ }^{82,130,135}$. The extent of the brain's vulnerability is not fully understood as how dangerous a second TBI could be. Second impact syndrome (SIS) is when catastrophic cerebral edema occurs after a repeated mild TBI ${ }^{12,15,24}$. SIS is mostly involved in sports-related activities and few in number but the mortality of those afflicted generates a huge cause for concern ${ }^{124}$.

\section{E. TBI mechanism}

Although TBI can be the result of a blast or penetrating load, the main focus of this thesis involved the brain's collision within an intact skull as a result of direct or indirect forces. Direct forces consist of any load to the skull itself that transfers energy to the brain. Indirect forces constitute brain stresses experienced as a result of inertial or acceleration loading. Direct forces cause both inertial loading and physical contact of the brain, e.g. head striking surface; but indirect forces only because inertial loading, e.g. impulsive head motion. Despite the type of force, common vectors of brain acceleration are linear and rotational. Early studies of TBI focused on linear accelerations, and good correlation between peak acceleration and peak pressures measured within brain surrogates was established in these models ${ }^{57,131}$. These observations lead several investigators to searching for a threshold and establishing injury tolerance curves. Later, more attention was given to rotational accelerations during head impact. Rotational acceleration causes brain tissue to deform more readily due to the anatomical position of the brain and its physical properties ${ }^{38}$. Regardless of the force's origin, the resulting linear and rotational accelerations of the brain and collision with the skull ultimately cause tissue deformation in the brain.

\section{a. Jerk}

The change in acceleration, or jerk, is noted as an important factor in TBI. This parameter is more than evident during the inertial change observed in closed-cranial TBl's. Many studies have been conducted to determine the relationship between head injury and jerk. The Wayne State Tolerance Curve was one of the more publicized early attempts to determine an injurious threshold. This curve was mostly 
derived from in vivo experiments involving several subjects and forms of trauma. This data was collected from cadaver falling-impacts, exposed dog brains subjected to air blasts, and human volunteers riding a rocket sled ${ }^{105}$. The most widely used function is found in federal motor vehicle safety standards ${ }^{104}$ called the head injury criteria focusing on the linear acceleration and impact duration during an impact. One of the newer mathematical models for TBI follows the coupled loading-rate hypothesis. Developed by Ivancevic, the model states that an impulsive loading coupled by several degrees-of-freedom. This loading is called an external Euclidean jolt and causes two forms of rapid discontinuous deformations in the brain $^{67}$. However, the complicated nature of brain injuries makes verification of these kinematic assessment functions difficult. To observe decelerations at impact, several biomechanical studies of helmeted collisions have been conducted. In particular, American football provides a high number of collisions ${ }^{16}$ and higher levels of TBI occurrences than other sports ${ }^{88}$. Original studies of helmeted collisions were initiated by Newman on behalf of the NFL. Using two recorded perspectives of known head injuries, the striking/struck head velocity was determined before and after impact and a resultant velocity could be determined ${ }^{107}$. The reconstruction of the helmet-to-helmet collision was reconstructed using two Hybrid-III adult male anthropomorphic test devices. Equipped with a configuration of accelerometers, the change in acceleration during impact could be recorded. These observations provided an initial range of values experienced by struck and striking players ${ }^{106}$. Newman et al. determined that a $50 \%$ probability of a mild TBI can occur from a $78 \mathrm{G}$ impact. Moving from photogrammetric analyses, the introduction of the Head Impact Telemetry system (HITS) allowed for immediate data capture during live play. Retro-fitted with a similar accelerometer configuration and telemetry unit, specialized helmets transmitted data to a local computer. Many studies have been performed to determine the values of impact seen at the collegiate and high-school level. Based on known events of concussion, Table 1 displays the collected data from several HITS studies and a mildTBI threshold of approximately $100 G^{18,33,46,112}$. Shockingly, these values can be observed in youth football as well ${ }^{34}$. Broglio et al. characterized biomechanical variables experienced during impact from high school football players. Using 35 players equipped with the HITS, linear acceleration, rotational acceleration, maximum jerk, impact duration, and impact force were collected during practices and games. Overall, these athletes experienced maximum head jerks of $10.38 \pm 9.92 \mathrm{~m} / \mathrm{s}^{3}$, impact durations of 
$8.99 \pm 3.01 \mathrm{~ms}$, and impact force of $1314.88 \pm 1023.36 \mathrm{~N}^{19}$. These investigations provide a better understanding of the biomechanical variables experienced during an impact, but the tissue response to such an impact is just as important.

\section{b. Strain}

Many biomechanical explanations of brain injury have been suggested from skull vibrations to pressure waves to cavitation; however, these explanations have yet to be confirmed through direct experimentation. One of the first studies used a human skull model filled with gel and noted the large shearing deformations caused by angular acceleration ${ }^{65}$. The inability to capture the data live left much to postulation as to what was occurring during the impact. Replicating animal studies producing clinically similar outcomes, quantitative studies of brain biomechanics were performed by Margulies and Meaney. The physical model simulation involved primate skulls filled with optically transparent gel mimicking the brain's mechanical properties. With the gel surface marked by a grid, a high-speed camera recorded the strains during impact. From these experiments, strains of $0.1-0.5$ and strain rates of $10-50 \mathrm{~s}^{-1}$ were determined to be capable of producing relevant forms of brain injury ${ }^{90,92}$. However, this data from gel cannot duplicate the complex structure of the brain and therefore its mechanical response. More recently, Hardy et al. tracked the brain displacement of cadavers using high-speed bi-planar x-ray imaging ${ }^{61}$. The spatial position of eleven neutral-density radio-opaque markers was recorded and produced the $\pm 5 \mathrm{~mm}$ nonpermanent displacement observed at 108G impacts. Because of the limited number of markers, spatial resolution was too low to determine tissue $\operatorname{strain}^{61}$. The first quantitative strain measurements from known inertial loadings was obtained by Bayly et $\mathrm{al}^{8}$. The tissue deformation with respect to a 2-D plane could be tracked using various new techniques in MR imaging and image analysis. Originally used to measure deformation of the heart, this MR method is capable of "tagging" the tissue and tracks its movement. The deformation must be repeated to accomplish a high temporal resolution and only a single line is observed for each deformation. Using a variation of the HARP method, the tagged MRI data is filtered and transformed to produce a value for every spatial point. Brain deformations of human and rat brains caused by low and high accelerations, respectively, have been established. Human volunteers subject to $2-3 G$ produced Lagrangian strains of $2-5 \%{ }^{8}$. The animal model observed maximum principal strains and strain rates greater than $20 \%$ and $40 /$ s, respectively, during the rapid, nonpermanent 
indentations of the P7 rat skull ${ }^{7}$. This study overlaps with actual measurements of brain tissue. Bain and Meaney determined an axonal injury threshold of approximately $20 \%$ strain for a guinea pig optic nerve ${ }^{3}$, while Prange and Margulies predicted an axonal injury of $30 \%$ strain in the infant pig ${ }^{114}$. Shreiber et al. collected cortical deformations during in vivo experiments of rat cerebral tissue and noted blood brain barrier breakdown is sensitive to maximum principal strains above $18.8 \%{ }^{127}$. Although many types of mechanical trauma can have various levels and different combinations of consequences, the general responses of CNS-cell injury are known.

\section{F. Cell response}

When severe, the tissue deformation can cause immediate, irreversible mechanical damage to the individual cells, like transmembrane proteins becoming disconnected with the surrounding extracellular matrix, neuronal processes severed from the soma, or the cell membrane itself being permanently compromised causing cell death ${ }^{52,100}$. This mechanical injury results in contusions, diffuse axonal injury of the parenchyma tissue, and intracranial hemorrhaging of its vascular tissue. Primary damage is defined by the extent of tissue damage. If focal; local tissue can be contused, contain hematomas, and increased intracranial pressure. Diffuse primary injury mainly consists of axonal, neural, and brain swelling injury. A more common form is diffuse axonal injury (DAI) whereby a widespread shearing of axonal tissue is present. Secondary damage results from cells experiencing lesser levels of primary damage and receiving deleterious signals from expired cells.

Secondary damage can develop some time period after the initial injury resulting in symptoms like further swelling, ischemia, and inflammation of the brain tissue. This secondary damage is the direct result from the primary damage and initiates an enzymatic cascade. This cascade has three main mechanisms: excitotoxicity, inflammation, and oxidative stress. Excitotoxicity is the constant overstimulation of post synaptic receptors by a neurotransmitter leading to cell injury and death. When the neuronal cell membrane is deformed, intracellular potassium rushes out into the surrounding extracellular space which results in release of many kinds of neurotransmitters like the excitatory amino acid glutamate. Glutamate binds to N-methyl-D-aspartate (NMDA), D-amino-3-hydroxy-5-methyl-4isoxazolepropionic acid (AMPA), and kainite ionic channels ${ }^{5}$. Binding to NMDA receptors activates 
depolarization of the cellular membrane and an influx of calcium ions. This ionic imbalance across the cell membrane causes ATP-dependent $\mathrm{Na}^{+} / \mathrm{K}^{+}$pumps to be highly active while attempting to restore this imbalance. The increasing metabolic demand quickly depletes intracellular storage, and the neuron undergoes anaerobic respiration leading to an accumulation of lactic acid. Possible local acidosis, increased membrane permeability, and cerebral edema have an increased likelihood when lactate accumulates $^{70}$. Meanwhile, neuro-supportive cells, like astrocytes and microglia, are inhibited from correcting extracellular glutamate concentrations by quinolinic acid, an EAA released by glutamate. Excitotoxicity becomes more disastrous when glutamate receptors and players from the inflammatory cascade intricately signal each other in a process called immunoexcitotoxicity ${ }^{13}$.

Many cells specific to brain tissue can produce inflammatory mediators, and cytokine receptors are consistently expressed at low levels throughout the central nervous system. Cytokines, like interleukins, that propagate inflammatory responses are released by astrocytes and microglia ${ }^{86}$. Dependent on the injury's severity and location, prostaglandins, other cytokines, and the complement system can be activated which leads to chemokine and cellular adhesion molecule involvement. Chemokine signaling stimulates recruitment of a host of leukocytes, cells, macrophages, and monocytes to these injury sites and activates microglia, as well.

Secondary damage from oxidative stress is focused on the presence of intracellular reactive oxygen species and free radicals. After TBI, levels of ROS and free radicals increase dramatically whereas their levels are low within normally functioning neuronal cells. Although having many ways of production, high intracellular $\mathrm{Ca}^{2+}$ concentrations increase the expression of enzymes that catalyze fabrication of free radicals (e.g. nitric oxide synthase and xanthine oxidase). Also, mitochondrial membrane disruption occurs from the $\mathrm{Ca}^{2+}$ influx and results in the degradation of the electron-transport chain leading to leaking of electron-reduced oxygen intermediates. Because oxidative stress can become self-propagating, cellular mechanisms designed to protect against oxidative damage can be overwhelmed. The resulting damage can be cellular membranes peroxidation, protein/DNA oxidation/nitration, and mitochondrial electron-transport chain disruption. However, secondary damage cannot only occur through the mentioned pathways but can also be mediated by over reactive enzymes. 
When depolarization and ionic channel activation from excitoxicity increases ionic concentration of $\mathrm{Ca}^{2+}, \mathrm{Ca}^{2+}$-dependent enzymes, like calpains and caspases, become activated and initiate necrotic and/or apoptotic pathways. Weakening mitochondrial and cellular structure, calpain-mediated necrosis begins cytoskeletal element degradation, like dendrite beading and microtubule disruption ${ }^{118}$. Caspases facilitate apoptosis through several pathways. Intrinsic pathways start from cellular organelle stress. After mitochondrial membrane depolarization and transition-pore formation, cytochrome $\mathrm{c}$ is released and interacts with the cytosolic protein Apaf-1 to formulate apoptosome. Apoptosomes recruit and activates an initiator caspase that triggers other caspases and begins an enzymatic cascade to apoptosis. For the myriad mechanisms that secondary injury can develop, many are unknown and can only be understood by its general concept. Many of these mechanisms are intricately connected and can couple one another. Although there is much to be discovered how these mechanisms interact and provide feedback during this injurious cascade, the mechanical initiator for these events is much desired.

\section{G. In vivo}

To investigate how these TBI's occur and develop, animal models have been extensively used and are now well established. Providing numerous insights, animal models have been the gold standard for understanding TBI. Although no one animal model can replicate the entire range of symptoms, selected symptoms can be investigated against set parameters. Proper establishment of an animal model for TBI can offer several forms of validity. While difficult to relate humans to animals, the similarity of the exhibited animal behavior and the specific human condition offers understanding in the mechanisms and predictability of injury.

The most common experimental models are divided by impacting-force origin. Direct-impact models can be designed to either impact the exposed brain (direct tissue deformation) or impact the exterior surface of the head (impact acceleration). Controlled cortical impact and the weight drop are two popular experimental models that follow the same direct-impact concept but differ on energy source. The controlled cortical impact model uses pressurized air that transfers energy to the intact dura of the restrained head. It was first developed by Lighthall and used ferrets as the subject ${ }^{79}$. This model offers the ability to control deformation time, velocity, and depth ${ }^{4,28,37,53}$. Also, it avoids the inherent risk of 
unintentional rebound injury from the impacter like what exists for the weight drop. The weight drop model relies on the guided free-fall of its solid impacter to the subject's head which can be prepared with a fixated metal plate or portion of the brain exposed. The simplicity of this method is not without faults as the impact velocity can vary and the risk of rebound still possible.

Indirect-impact models allow linear and/or rotational acceleration from a force not applied to the head (instead possibly applied to the whole body). Due to the occurrence and nature of motor vehiclerelated TBI's, many variations of applied inertial acceleration to animal models have been established. . Ommaya et al. used primates as their main subject because of their gyrencephalic brain structure and relatively large brain to body mass ratio ${ }^{100}$.

For all that in vivo models have provided, these models can be expensive, complicated, and raise ethical concerns. Consistent shelter, resources, and minimal stressful conditions are required not only for reduction of behavioral variance but mandated by organizations, like IACUC. Often times, a particular breed of animal is required to reduce the amount of genetic variation between subjects. Prior to impacting, these animals must be properly prepared before impact so the impact location can be consistent amongst all the subjects requiring anesthesia and restraints. Not only does the animals' conditions be reproducible but impacting parameters such as impact velocity or pressurized pneumatic force. For observation of the response, some outcome is measured rather than the actual mechanical stimulus because of the trouble controlling and verifying internal tissue and cell level biomechanics ${ }^{100}$. Coupling these models with micro dialysis provides insight into concentration levels of specific ions or biomarker but also add to the complexity required for all components involved to work in unison.

Indirectly, many investigators using these animal models miss the opportunity to record the biomechanical motion of the head. Although a mechanical stimulus was used to initiate the injury, no consideration for the deceleration-time profile during impact was utilized. Lastly, the use of animals, especially those with a higher cognitive capability, adds to the ethical dilemma of reasonable experiments and required sacrificial subjects versus ethical responsibility. As an adjunct to in vivo models, in vitro models avoid these pitfalls.

\section{H. In vitro}


Although in vitro models forfeit the realistic outcomes from a complex biological system (e.g. systematic inflammation or ischemia), they can provide a more controlled and reproducible platform for study. No one in vitro model can completely replicate the TBI sequelae present in vivo models, but they do offer insights into the many mechanisms that occur during and well after injury. By controlling the biomechanics of injury, the effects of injury severity can be determined by observing the resulting pathobiology of the cell culture. The first published model of CNS injury incorporated a rotating stir-bar to inflict trauma to a $1-\mathrm{mm}^{3}$ sample of rat cortex in a nutrient medium ${ }^{44}$. Although the study did not address the cellular mechanics from the injury, they did determine that neurons were found to be the most prone to injury followed by astrocytes. Using more relevant forms of TBI-like insults, today's mechanical stimuli range from compressive forces to transection ${ }^{73,98,100}$. One popular in vitro injury is the substrate stretch model where cells or tissue is adhered to a thin, flexible substrate (e.g. silicone rubber) which is stretched thereby stretching the cells or tissue attached. This model tries to replicate the brain tissue deformation that occurs in head impacts whose strain values were established by physical model simulations that replicated clinically relevant, post-injury outcomes from in vivo studies ${ }^{90,92}$. More recently, live in vivo capture of the strain field generated by an impact was further validated by Bayly and company at Washington University. Several aspects of stretch and its application have been developed. Kit Parker's group from Harvard developed a uni-axial substrate stretcher to investigate how neuronal integrins interacted with the protein covered substrate and determined the level of primary and secondary damage $^{62}$. Morrison developed a biaxial stretching bioreactor initiated by a rigid hollow cylindrical indenter pressing into the flexible substrate. This bioreactor experimented with region-specific, brain tissue's reaction to various levels of strain magnitudes and strain rates ${ }^{97}$. Another form of cell injury used is acceleration/deceleration. Lucas and Wolf developed a bioreactor that applied accelerations as great as $200 \mathrm{G}$ to a flask of cultured cells using an impacting pendulum ${ }^{85}$. They determined cellular death dependent on cell position upon impact (tangential versus normal) and frequency of impact.

\section{DESIGN SUMMARY}

Our design goal was to combine the concepts of substrate strain and inertia loading as seen in invitro and in-vivo models, respectively. We wanted our device to have the capability of applying impact decelerations up to $200 \mathrm{G}$ 's and biaxial strains up to $25 \%$ to multiple sterile cell culture samples all while 
occupying a small footprint that would be compatible with a university research lab. These parameters were determined by the preceding literature. Although, there has been great work done with an in vitro acceleration-based model, we believe further investigation into the possible subtle cellular changes due to this inertia loading is warranted. Not only do we want to investigate the effect of rapid jerk, but the effects of tensile strain and impact duration to a two-dimensional culture of cells. The simplicity of our design allows easily manipulation of the desired injurious parameters while allowing for multiple samples to be treated with the same jerk but possibly different strain magnitudes.

\section{FABRICATION}

The three major components of our bioreactor consist of a movable drop-shuttle, vertical rod, and impact base (Fig.1). The drop shuttle's motion is constrained to only one axis by a vertical, steel rod $(3.81 \mathrm{~cm}$ OD) which is press fit into an aluminum plate $(30.5 \mathrm{~cm}$ by $30.5 \mathrm{~cm}$ by $2.54 \mathrm{~cm})$. A single-axis accelerometer (ADXL193, SparkFun Electronics, Boulder, CO) is attached to the top of the shuttle and connected to a PC via a 16 digital acquisition card (NI USB-6211, National Instruments, Austin, TX). A custom designed LabVIEW (SP1 2011, National Instruments, Austin, TX) program was used to convert accelerometer voltage output $(0-2.5 \mathrm{~V})$ into values of gravitation (G: the ratio of the current shuttle's acceleration to the gravitational constant of earth), as well as plot and store the shuttle's impact profiles (Fig. 2).

The drop shuttle consists of a custom-fabricated, top and bottom aluminum plates $(17.78 \mathrm{~cm}$ by $17.78 \mathrm{~cm}$ by $0.64 \mathrm{~cm})$ sandwiching a thicker polypropylene layer $(17.78 \mathrm{~cm}$ by $17.78 \mathrm{~cm}$ by $1.7 \mathrm{~cm}$; Fig.3A). A linear mount bearing is incorporated into the top plate to facilitate smooth and near-frictionless translation of the shuttle along the guide rod. The middle layer contains a circular ring of cylindrical holes that are designed to carry six independent cell culture inserts. The drop layers of the drop shuttle are held together with nonpermanent fasteners (tapered head bolts and wing nuts). The shuttle is disassembled to load and unload the cell inserts into the shuttle.

To carry cells within the shuttle, sterilizable cell culture inserts were fabricated. Seen in the upper figure the walls of the well-shaped inserts were fabricated from polypropylene. The base of the cell culture inserts is formed by a PDMS membrane which is held in place by a press fit silicone O-ring. The PDMS membrane offers a sterilizable, flexible, and optically translucent substrate; so cell attachment and 
viability can be easily observed with a microscope. These inserts are sized to fit into a six well plate during cultivation then are transferred to the shuttle for impact testing (Fig. 3A).

The bottom plate of the shuttle has a circular series of cylindrical holes designed to hold six individual Teflon indenters directly underneath the cell culture inserts. To apply a biaxial strain to the PDMS substrate, cylindrical Teflon indenters are inserted into holes located along the bottom of the shuttle. Each of the six indenters is aligned with a separate cell culture insert (Fig. 3B). When dropped, contact of the shuttle with the impact base drives the indenter into the flexible base of the cell culture inserts (Fig. 3C).

\section{METHODS}

\section{A. Characterizing Deceleration}

The shuttle's impact deceleration was measured by a $\pm 250 \mathrm{G}$ single-axis accelerometer (SparkFun Electronics) fastened in the normal direction to the top shuttle plate. The accelerometer was connected to a PC computer via a 16 bit analog to digital card (National Instruments). The accelerometer's output signal was captured $(10 \mathrm{kHz})$ by a custom data collection and analysis software (LabVIEW). After the data acquisition virtual instrument (VI), the signal was passed through a Trigger and Gate VI and the triggered signal split between a waveform chart and a "write to measurement file" VI. If the signal rose above a specific threshold from the baseline, the Trigger and Gate VI would capture the triggered signal and send that triggered signal to be displayed and recorded. The measurement file of the triggered signal is exported to Excel for graphical representation. The mean deceleration-time profile was determined from several recorded drops $(n=5)$ at heights ranging from 10 to $100 \mathrm{~cm}$. The effect of impact base stiffness was also explored. Three open-celled foam (polyurethane) pads with thicknesses (3.18, 6.35, and $9.53 \mathrm{~mm}$ ) were individually positioned on top the impact base. Impact of the shuttle with the padded base was recorded to for the deceleration-time profile dependent on the impact base.

\section{B. Characterizing Biaxial Strain}

Indenter heights were first established by theoretical geometric analysis using the following Lagrangian strain equation as previously used by Morrison et al. ${ }^{97}$ 


$$
E=\frac{1}{2}\left[\left(1+\frac{h-c}{r}\right)^{2}-1\right], \text { where } h=\left(c^{2}+d^{2}\right)^{0.5}
$$

After determining the desired biaxial strain, the required indenting height was determined from this relationship. Experimental biaxial strain of the membrane versus indenter height was characterized optically. Using glass microspheres $(30-50 \mu \mathrm{m})$ as markers, pre and post indentation images were obtained (Fig. 4) with the aid of a light microscope and digital camera (Southern Tech). Using these images and a custom designed finite strain analysis program (MATLAB), the normal and shear strain magnitude for indenters ranging in height $2 \mathrm{~mm}$ to $6 \mathrm{~mm}$ was calculated from several triangular arrangements $(n=3)$. To check for uniformity, the strain from the same indenter was also measured at different locations $(n=3)$ along the radial axis of the cell insert bottom. This experimental procedure was adapted from Lee et al. ${ }^{77}$

The two dimensional strain calculations were determined using the Lagrangian strain tensor, $\mathrm{E}_{\mathrm{ij}}$, and solving the following equation

$$
d s^{2}-d S^{2}=2 E_{i j} d X_{i} d X_{j} \quad(\mathrm{i}, \mathrm{j}=\mathrm{c}, \mathrm{r})
$$

The two-dimensional strains are referred to as the circumferential (c) and radial $(r)$ axes. dS is the length between the markers pre-indention, $d s$ is the length between the markers post-indention, and $d X_{c}$ and $d X_{r}$ are the two-dimensional components of the segment length along the two axes pre-indention. The two normal strains, $E_{c c}$ and $E_{r r}$, symbolize the stretching of the membrane in the circumferential and radial directions, respectively. The shear strain, $E_{c r}$, measures the resulting shear strain due to any angle change between the circumferential and radial axes, like twisting. Therefore, a truly equibiaxial strain will have the normal strains equal $\left(E_{c c}=E_{r r}\right)$ and the shear strain null $\left(E_{c r}=0\right)$.

The MATLAB code responsible for determining the Lagrangian strains of each triangular arrangement required the coordinates of the individual markers in the pre- and post-indention images (Appendix A). Their coordinates in the image were determined by the available ImageTool function and recording the pixel coordinates of the marker's center. Entering the coordinate points allowed for simple matrix algebra 
to determine the resulting Lagrangian strains along the two axes. The data was exported to Excel to determine the mean, standard deviation, and uniformity of the Lagrangian values.

\section{Preparation of Cell Culture Insert}

Prior to assembly, all cell culture insert components (polypropylene insert, polydimethylsiloxane membrane, and rubber O-ring seal) were washed in 1\% (w/v) detergent powder (Alconox, White Plains, NY) solution for 30 minutes at room temperature with agitation to remove all oil and debris from the manufacturing process. The components were then washed in a $50 \%(\mathrm{v} / \mathrm{v})$ ethanol solution in deionized water (DI) for 20 minutes, and then rinsed in DI water for an additional 20 minutes to remove any residual detergent. Once washed; the cell culture inserts were manually assembled; excess PDMS was trimmed; and all components sterilized at $135^{\circ} \mathrm{C}$ for 10 minutes using a bench-top autoclave (2340M, Tuttnauer, Jerusalem, Israel). Sterile cell culture inserts were transferred into a six-well plate for protein coating.

To promote cell adhesion, the base of the cell culture insert (the PDMS membrane) was coated with a rat-tail collagen solution (Life Technologies, Grand Island, NY; 3mg/mL) or fibronectin solution (Life Technologies) for PC12 or C8-D1A, respectively. For PC12, the diluted collagen solution was allowed to air-dry within the cell culture insert overnight while in a sterile, biological safety cabinet, so a $16 \mu \mathrm{g} / \mathrm{cm}^{2}$ collagen film formed onto the insert base. After air-drying, collagen-coated inserts were rinsed three times with sterile PBS to remove any collagen that had not physisorbed to the PDMS membrane. For C8-DA1, the fibronectin solution $(20 \mu \mathrm{g} / \mathrm{mL})$ sat in the cell culture insert overnight while stored in a $4^{\circ} \mathrm{C}$ refrigerator. Prior to C8-DA1 seeding, the fibronectin solution was removed, and the cell culture inserts rinsed three times with sterile PBS.

Commercially purchased rat pheochromocytoma (PC12) (CRL-1721, ATCC, Manasas, VA) cells were expanded from frozen stocks in RPMI 1640 media (Life Technologies, Grand Island, NY) supplemented with 10\% (v/v) heat-inactivated horse serum (Life Technologies), $5 \%(\mathrm{v} / \mathrm{v})$ fetal bovine serum (Life Technologies), and 1\% penicillin-streptomycin (Life Technologies) ${ }^{56}$. Following expansion

cells were seeded onto collagen-coated cell culture inserts at a concentration of $2.5 \times 10^{4} \mathrm{cell} / \mathrm{cm}^{2} 55$. Once seeded, PC12 cells were maintained in the above described culture media, supplemented with $50 \mathrm{ng} / \mathrm{mL}$ nerve growth factor (NGF, Peprotech, Rocky Hill, NJ). Stimulation by NGF causes PC12 cells to 
differentiate into a neuronal phenotype characterized by the growth of numerous neuronal-like processes. The cells were cultured in a humidified incubator $(3110$, Thermo Fisher Scientific, Mariette, $\mathrm{OH})$ at $37^{\circ} \mathrm{C}$ and $5 \% \mathrm{CO}_{2}$. Media was exchanged every 2-3 days until testing.

Commercial purchased astrocytes (C8-DA1; CRL-2541, ATCC, Manasas, VA) were expanded from frozen stocks in DMEM: F-12 media (Life Technologies) supplemented with $9 \%$ fetal bovine serum, 0.9\% L-Glutamine (Life Technologies), and 0.09\% Gentamicin. C8-DA1 was seeded at $7.5 \mathrm{X} 10^{5} \mathrm{cells} / \mathrm{mL}$ in the cell culture inserts and incubated for two days until testing.

\section{Cell Impact Testing}

After 7-9 days in culture, cell inserts were loaded into the drop shuttle and treated with one of the following impact conditions (three samples per condition),

1. High Impact: $100 \mathrm{G}$ of peak deceleration combined with $10 \%$ strain

2. Low Impact: $50 \mathrm{G}$ of peak deceleration combined with $5 \%$ strain

3. Control: no impact or strain

Immediately following impact, treated and control cultures were rinsed in complete media to remove any free or loosely attached cells, fixed in $4 \%$ paraformaldehyde, permeabilized (Triton X-100, $0.1 \%$ in PBS), and stained with DAPI to identify cell nuclei. Tested and control cell culture inserts were microscopically imaged (thirteen fields per insert at 100X magnification). All images were digitally captured, and cell counts were measured using image analysis software (ImageJ). Average cell counts for each treatment condition relative to controls were used as a measure of cellular survival.

\section{RESULTS}

Using the mounted accelerometer and LabVIEW program, the impact profile of the shuttle during its initial contact with the base was recorded. The average profile of five runs was determined for specific drop heights and pad thicknesses (Fig. 5). Repeatability of the impact profile was high as the pooled standard deviation of the five runs at any time point was low; for example, the impact profiles on the $3.18 \mathrm{~mm}$-thick pad had a pooled standard deviation of $1.34 \times 10^{2} \mathrm{G}$. The impact profile was a symmetrical slope with a sharp rise and fall with small oscillations at the end. From the impact profile, certain 
characteristics were especially noted: maximum deceleration, maximum jerk, and impact duration. Maximum deceleration was noted as the positive peak of the slope, maximum jerk as the greatest positive derivative of the impact profile, and the impact duration was the width of the impact profile at half of the maximum deceleration, called the half-peak width (Fig.6). The maximum deceleration increased with increasing drop height (Fig. 7A). From the impact profiles, the maximum jerk (G/s) was determined by deriving the mean impact profile specific to impact base and drop height (Fig.7B). By only changing the padding's thickness, the damping caused by the thicker pads required higher drop heights to create the desired range of deceleration magnitudes up to $300 \mathrm{G}$ (Fig. 7C). The impact duration of the profile was increased by increasing the base's thickness of the foam padding (Fig. 8A). The impact duration increased from approximately 1 to $3 \mathrm{~ms}$ by increasing the foam pad thickness from 3.2 to $16.0 \mathrm{~mm}$ (Fig.8B). The relationship of impact duration to padding thickness was a sharp, positive rise that abated to a potential maximum of $3 \mathrm{~ms}$ at $16.0 \mathrm{~mm}$. No impact profiles were recorded for thicknesses greater than $16.0 \mathrm{~mm}$ at a $200 \mathrm{G}$ impact. The resulting spring constant was determined from the impact duration and mass of the assembled shuttle $(2.0684 \mathrm{~kg})$. The spring constant of the impact base decreased with pad thickness (Fig. 8C). Assuming an ideal mass-spring system, the natural frequency of the base was determined from the impact duration of the impact profile which was dependent on the pad thickness. When the time interval is scaled to a typical time frame recorded for HITS, the impact profile is similar to profiles observed in helmeted collisions (Fig.9) at approximately 90G impacts ${ }^{19}$.

The Lagrangian strain of the substrate increased with increasing indenting height (Fig. 10). The strain ranged from 5 to $25 \%$ with indenting heights of approximately 2 to $5.5 \mathrm{~mm}$. The Lagrangian strains of the circumferential and radial axes for each indenter were compared for equality. Performing ANOVA for each indenter determined that these Lagrangian strains produced were not significantly different $(p<$ 0.5). The strain values across the stretched membrane were checked for uniformity by tracking three separate triangular arrangements per position at three positions along a radial axis (Fig. 11). An ANOVA of the strain values compared to each position along the radial axis confirmed that they were not significantly different. 
After samples for the treatments and control $(n=3)$ were fixed, stained, and counted; our preliminary assay displayed a varied responses in cell attachment with increasing deceleration and biaxial strain (Fig. 12). Though the mean count of $P C 12$-attachment appears to decrease, no statistical significance could be established from the control. The C8-DA1 attachment from the $50 \mathrm{G} / 5 \%$ impact was significantly different from the control based on our student t-test $(p<0.05)$. No conclusive result could be determined from our assay except for the device's biocompatibility.

\section{DISCUSSION}

The presented bioreactor has been developed, characterized, and validated for further in vitro studies using TBI-like parameters on 2-D cell cultures. Developing a bench-top bioreactor capable of holding multiple, sterile cell cultures allows for greater insight and control of our two injurious inputs than could be accomplished with an in vivo model. To our knowledge, it is the first to combine deceleration and strain forms of cell injury into one in vitro model. The lack of deceleration models in in vitro studies and lack of recorded decelerations during in vivo impacts gives further cause for investigation of inertialoading effects. The addition of strain during impact better replicates the in vivo conditions quantitatively captured during deceleration impacts and resulting tissue strain ${ }^{7,8}$.

The bioreactor was successfully characterized for bi-axial strain dependent on the height of the indenter, as well as the maximum deceleration magnitude dependent on drop height and impact base material. To show its potential in cell injury, the bioreactor was validated by tracking the level of cell attachment of two cell lines (PC12 and C8-DA1) dependent on maximum deceleration and biaxial strain. With their neuronal-like phenotype, the resulting decrease in PC12 cell attachment with increasing maximum deceleration and biaxial strain imitates the known susceptibility of neurons ${ }^{44}$ and their processes $^{62}$ to substrate disconnection. However upon closer inspection, the data proved inconclusive to develop a correlation using our simple assay of cell attachment dependent on inflicted strain and deceleration. Although no conclusive result could be determined, biocompatibility was validated. Our bioreactor is fairly different from other bioreactors, but its sensitivity allows the same range of values used in various other bioreactors, in vivo models, and physical simulations. 
Our bioreactor is one of the few in vitro studies to focus on the inertia loading during impact. Bottlang et al. developed a bioreactor that implemented inertia-driven shear strain. Using organotypic cultures adhered to microporous membranes, they quantified cell death dependent on impact velocity of the inertia-loading module against a damper. The propidium iodide staining provided the intuitive correlation that higher impact velocity increased cell death. Although the shear insult of the hippocampal cultures was determined, the data collected could have been relatable if the resulting jerk been disclosed. The only other in vitro model using inertial loading is the previously mentioned pendulum-impacting bioreactor from Wolf and Lucas. With a maximum deceleration of 220G per impact, the pendulum-based bioreactor has a range that easily fits in the possible range of our bioreactor. Although currently limited by the $\pm 250 \mathrm{G}$ accelerometer, the available length of the approximate $1.20 \mathrm{~m}$ rod has the potential to deliver >300G impacts especially when considering the choice of the impact base material; the use of the $3.18 \mathrm{~mm}$-thick pad (firm) has a sensitivity of approximately $7 \mathrm{G}$ for every $1 \mathrm{~cm}$ increase in drop height (Table 2). The sensitivity of Wolf and Lucas' bioreactor was capable of determining neuronal cell death dependent on cell orientation on impact and frequency of impact. They determined that the tangentiallypositioned cells were more sensitive to impacts than normally-positioned impacts. After 10 impacts (2200G accumulation), $45 \%$ of tangentially-impacted neurons were dead as compared to only $12 \%$ seen in normally-impacted cultures. Erythrosine B dye exclusion method, marked neuronal death from tangential impacts was not noticed until 5 impacts (1100G accumulation) had been reached. Neuronal, morphological changes after 10 tangential impacts (2200G accumulation) consisted of swollen somas, prominent nuclei, and nuclear shifting. Loss of soma adhesion to the polylysine-coated flasks only occurred to $6 \%$ of the dead neurons. The preliminary studies of our bioreactor only used a DAPI stain of cells attached to the membrane. We observed a loss of soma adhesion from collagen-coated siliconerubber substrates occurring in the mean count of PC12 cells but no significant difference with the control could be established (Fig. 12). Wolf and Lucas also noted that observed glial and other support cells rarely died after impacts (less than $2 \%$ of selected areas). Interestingly similar, C 8 -DA 1 soma adhesion was unfazed by any impact and strain inflicted by our bioreactor, though some error was present in the samples subject to $50 \mathrm{G} / 5 \%$ parameters. Although Wolf and Lucas observed no morphological changes in glial cells, they only tracked visible changes from Polaroid micrographs. 
Viano et al. established one of the few weight drop in vivo models that includes measurement of the animal's head using a mounted accelerometer. Many animal studies lack this important parameter when determining histological differences dependent on the impact velocity rather than the resulting head acceleration. Although difficult to relate the sensitivities of an invivo model to our in vitro model, the results of brain injury from known inertia loads is very useful. Using the heavier of the available weight drops, considerable increase in the percentage of brains injured occurred when the100G threshold was surpassed. This difference became even greater when the impact was repeated. When the animal response was scaled to a human response, the resulting $131 \mathrm{G}$ from a single impact of the $100 \mathrm{~g}$ impacting-mass caused approximately $78 \%$ of the rat brains to be injured ${ }^{137}$. Focal contusions and subdural hemorrhages were mostly observed in rat brains treated with a single impact at human-scaled accelerations greater than $131 \mathrm{G}$. Very little deceleration data is collected from other in vitro and in vivo models as compared to the numerous studies focusing on the consequences of mechanical strain to CNS-cells.

Although substrate stretch studies are prevalent, comparing the results from our bioreactor to others is difficult due to differences between the way strain is generated and types of cells that were cultured. The limitation of the biaxial strain inflicted by our bioreactor is limited by the height of the cell culture insert $(\sim 17 \mathrm{~mm})$. Based on a linear approximation of the Lagrangian strain-indenting height relationship (Fig. 10), we have a sensitivity of approximately $4 \%$ Lagrangian strain per $1 \mathrm{~mm}$ increase in indenting height. Therefore, we could possibly have a Lagrangian strain of $63 \%$ subjected to cultured cells within the cell insert, assuming the very elastic, silicone-rubber membrane is maintained during the rapid indentation. The range of the indenter-based bioreactor from Morrison et al. has a similar homogenous strain field generated but subjected to organotypic tissue cultures. Cell injury was observed as low as $10 \%$ Lagrangian strain to their $3 \mathrm{D}$ culture, but only to approximately $30 \%$ of the culture ${ }^{96}$. Ellis et al. treated a primary culture of astrocytes but applied strain using a pneumatic pulse ${ }^{43}$. Applying a heterogeneous, biaxial strain field, they observed morphological change and swelling at $31 \%$ membrane deformation, but this was their lowest deformation tested. Kit Parker's group noticed focal swelling in their cortical neuron cultures as soon as $10 \%$ strain of the substrate, but the strain was only applied in one direction unlike our bioreactor ${ }^{62}$. Geddes observed a marked calcium response at their lowest biaxial, 
homogeneous strain of $10 \%$, but a permanent change in the signal was noticeable for strain magnitudes

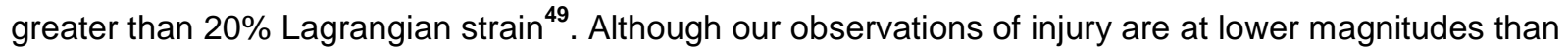
others, the combination of biaxial strain and deceleration treatment to our samples must be considered.

Two limitations concerning strain is its relevancy to TBI. It is generally understood that the brain's tissue response to mechanical loading is complicated and non-uniform. Compression, shear, and tensile forces are all occurring at the moment of impact. Currently our model only subjects cultures to tensile strain. One alternative method our bioreactor could subject cells to compressive forces by pre-stretching the cultures with an attached indenter while the cell culture inserts are positioned in the shuttle. Designing a mechanical trigger for the indenter's position within the cell culture insert, the cell culture inserts could experience compressive strain upon impact when the trigger would release the indenters from pressing into their cell culture inserts. An additional component to the shuttle and base would be required if this design goal was desired during impact decelerations.

The other limitation is the undetermined strain rate of the membrane during impact. This biaxial deformation is directly influenced by the impact deceleration and currently cannot be individually altered using this bioreactor. Both strain magnitudes and strain rates of certain ranges were considered crucial for TBI in the physical model simulations of Margulies and Meaney ${ }^{90,92}$. However, the exact role of strain rate during injury is still uncertain. Morrison's group used their indenter-based bioreactor on hippocampal tissue cultures subjected to strain magnitudes and strain rates from 0.05-0.50 and 0.1-50 $\mathrm{s}^{-1}$, respectively. They tracked the temporal development of cell death dependent on strain, strain rate, and anatomical region of the tissue culture. Using a three-way repeated measures ANOVA, strain rate was determined as a nonfactor in cell death ${ }^{25}$. Using their pneumatic pulse-based bioreactor, Geddes et al. subjected cortical neurons to heterogeneously applied biaxial strain to determine plasma membrane permeability ${ }^{50}$. The percent of exclusion dye intake was dependent on the applied strain rate, strain magnitude, and molecular size. If strain rate of the membrane was desired, theoretical analysis of the membrane strain dependent on impact deceleration could be mathematically determined and using a high-speed camera and the Digital Image Correlation technique would verify the strain rate of the membrane ${ }^{99}$. 
The following limitations are discussed for future studies. To better this bioreactor for a TBI condition, the impact duration must have greater range and control; and a more relevant cell type and assay for assessing TBI-like trauma must be tested. According to older studies of cadaver and animal skull impacts, the impact durations experienced by the cell culture inserts is very comparable to others. Goldsmith's physical simulation of cadaver skull impacts noted a range from 3 to $7 \mathrm{~ms}$ at low velocity impacts; higher impact velocities reduced this duration slightly ${ }^{109}$. Ommaya performed in vivo impacts on live primates and recorded impact durations ranging from 1.5 to $24 \mathrm{~ms}$ at various accelerations and vectors $^{108}$. Genneralli reports impact durations of less than $200 \mathrm{~ms}$ during TBI episodes without skull fracture $^{52}$. The impact duration of this bioreactor ranged from 1 to $3 \mathrm{~ms}$ and is dependent on the base's hardness and geometry. To gain greater control of this parameter, an additional component is being designed to the impact base (Fig.13). A platform is positioned over the impact base and has an arrangement of springs between the platform and base. Controlling the number of springs of specified spring constants would allow for a greater range of impact durations possible.

The other limitation of our bioreactor is the biological component. The experiments conducted with the PC12 cells established the biocompatibility of the bioreactor and demonstrated cell-attachment dependent on deceleration/strain magnitudes. PC12 cells have been used as models for neurotrauma of primary and secondary injury ${ }^{\mathbf{1 0 2}}$. However, PC12 cells are an immortalized cell line derived from nonneuronal tissue, therefore it's genetic/protein expression cannot replicate the response of neuronal cells to mechanical stimuli. Due to the nature of the cell line, further use of PC12 is unnecessary for future goals with the bioreactor. To study the aspects of TBI, the inclusion of CNS-type cells is required. One cell culture for consideration is a primary culture of astrocytes. As mentioned previously, astrocytes play an imperative role in maintaining neuronal function and health during normal and injurious conditions. Astrocyte cultures have been studied for its response to primary and secondary injury ${ }^{35,64,74,119}$; but none to our knowledge using the combined parameters of deceleration magnitude, impact duration, and biaxial strain. While primary damage in the form of soma attachment was the main injury assessment of the PC12, future studies will focus on astrocytes response through secondary response. Regardless of the mechanisms initiated by the cell, primary damage will initiate a response that can be tracked by genetic expression or biomarker concentration. 
This brings us to another future goal for this bioreactor concerning its biological component: conduct experiments with CNS-type cell (astrocytes) and determine a dose-sensitive marker of injury. Whether the injury marker is established or fairly new, the level of expression would be observed against the three input parameters of the bioreactor: strain, deceleration magnitude, and impact duration. Although the response from a two-dimensional, primary culture would be far from an in vivo response, clearly observing the cellular response from a crucial CNS cell will gain further understanding of the mechanical stimuli initiating it. Several older and newer markers of CNS trauma are considered for observation.

Intermediate filaments consist of an abundant family of cytoskeletal proteins and play a supportive role in cellular structure and response to mechanical forces. Out of all types of intermediate filaments, astrocytes can express vimentin, nestin, and glial fibrillary acidic protein (GFAP) ${ }^{111}$. These intermediate filaments have been extensively studied because of their close relation to reactive astrocytes. In vivo studies determined the vast majority of astrocytes are nonreactive and completely devoid of intermediate filaments under normal conditions. In particular, GFAP has been the focus of many studies during injury. The upregulation of GFAP has been an established hallmark of reactive gliosis and has been present in experiments inducing gliosis from stab wounds, cryogenic lesion in the brain, and toxic substances. GFAP mRNA has also been associated with scrapie, Alzheimer's, and CreutzfeldtJacob disease. One in vivo study using a weight drop method investigated GFAP's role in injury ${ }^{103}$. They determined mice deficient in GFAP were hypersensitive to a severe head injury when compared to their wild-type counterparts. A histological study on reactive astrocyte morphology revealed that GFAP ${ }^{-/}$ murine astrocytes had fewer but spiral, long processes which may attribute to the weakness toward acute stress $^{103}$. GFAP has a prominent relation with injury that could be further investigated using this in vitro model of injury.

Although the most widely used astrocyte marker, GFAP is not present in all astrocytes or every part of the astrocyte ${ }^{22}$. For immunohistochemical staining, it does not label all processes of the astrocyte and preferentially expresses more in astrocytes derived from white matter than gray matter. GFAP is not expressed in some types of astrocytes as well. Aldh1L1 is a fairly new marker discovered to be expressed 
throughout the entire astrocyte. A recently formed transcriptome database provided excellent characteristics of Aldh1L1 for astrocyte labeling ${ }^{23}$. Not only does it better label the entire astrocyte but is not expressed by oligodendrites or neurons. Although there are relatively few studies investigating Aldh1L1 during injury, Aldh1L1 (and GFAP) are upregulated during reactive synaptogenesis ${ }^{81}$. They also have higher mean levels of expression in patients suffering from mental disorders such as schizophrenia, bipolar disorder, and major depression ${ }^{6}$. With Aldh1L1 being such an astrocyte-specific marker, its possible relationship with injury could provide more accurate injury sensitivity than observing GFAPexpressions.

Intermediate filaments are not the only cytoskeleton protein to be considered. Using mass spectrometry, many actin-related proteins were found after injury in an in vitro transection-injury model ${ }^{84}$. Actin itself was not found but many other proteins closely associated with the cytoskeleton were in the extracellular medium. Two actin-associated proteins, ezrin and moesin, were specifically noted for their presence after astrocyte injury. Ezrin, radixin, and moesin (ERM) are major organizers of specialized membrane domains within the cell. Without the special coordination of processes across the cell membrane, signal transduction would be impossible. Mostly absent prior to injury, finger-like expressions of ezrin and moesin in injured astrocytes appeared. To verify in vivo, adult mice were subject to a CCl (controlled cortical impact) and the cortical brain tissue was examined through Western blot analysis and immunofluorescence staining. Using these observations, Loov et al. demonstrated that astrocytes expressed ERM proteins (ezrin, radxin, moesin) in processes and vesicle-like structures after $\mathrm{CCl}^{84}$. A previous study noticed the same high expression of ezrin and moesin after a cryogenic brain injury ${ }^{93}$. The reason for their appearance is not known, but they seem to have a role in engulfment ${ }^{83,84}$. Using immunostaining with specific antibodies, ERM proteins were strongly expressed around engulfed dead cells $^{84}$. As mentioned earlier, astrocytes do have the ability to phagocytize therefore possibly preparing the reactive astrocyte for this task.

Endothelin (ET) is a 21-amino-acid bicyclic peptide which is considered to be an effective vasoconstrictor. Normally absent in quiescent astrocytes, activated astrocytes contain endothelin in a wide range of CNS pathologies, including traumatic injuries ${ }^{59,120}$. Using a cryogenic injury, Siren et al. 
found a direct correlation between GFAP expressed levels, morphology changes, and ET-1 expression in rat brains ${ }^{128}$. Although endothelin can come from other sources (e.g. damaged blood vessels), many studies have reported activated astrocytes producing, storing, and secreting endothelins ${ }^{40,41,87,133}$. Expressed on astrocytes, endothelin receptors offer a positive feedback loop for the secretion of more endothelin, as well. It has been reported that ET-1 can stimulate the proliferation of astrocyte cultures at nano-molar concentrations ${ }^{26,69,76,87}$. ET-1 production is known to be initiated by mechanical stimuli but the majority of these studies only consider tissues involved in the circulatory system. Ostrow et al. has observed ET-1-positive reactive astrocytes in the mechanically deformed periphery of CNS trauma. His group has hypothesized that mechanical stress may regulate endothelin in astrocytes. Subjecting primary cultures of astrocytes, cyclic stretching created a dramatic increase in ET-1 production and secretion ${ }^{110}$.

One of the most extensively researched biomarkers for TBI is $\mathrm{S} 100 \beta$. This calcium-binding protein is known as one of the most present in the brain and can be found in astrocytes, oligodendrocytes, and Schwann cells. Although detectable in serum and cerebrospinal fluid after TBI, it's brain-specificity is questionable since $S 100 \beta$ is present in adipocytes, chrondrocytes, and melanoma cells. Several studies indicate that $\mathrm{S} 100 \beta$ serum levels have high sensitivity but poor specificity for moderate to severe TBI diagnosis ${ }^{116,121,138}$. However, a recent clinical study characterized tissue specificity of $S 100 \beta$ and $S 100 \beta$ serum levels form extracranial sources ${ }^{113}$. After analyzing 200 subjects, Pham et al. determined that extracranial sources did not affect $\mathrm{S} 100 \beta$ levels in serum, and the brain released a particular species of S100ß, B-B homodimer, not readily present from other sources ${ }^{113}$. Bazarian et al. found that using the combined use of serum $S 100 \beta$ and apoA-I values greatly increase classification accuracy for determining mild $\mathrm{TBI}^{10}$. ApoA-I is an apoliprotein created in the liver and small intestine and is a negative marker for inflammation and recently noted as a potential biomarker for mild $\mathrm{TB}^{14,117,136}$. Although significant subgroup variation in age and race existed, $\mathrm{S} 100 \beta$ is best at predicting abnormal initial head CT scans after mild TBI ${ }^{10}$.

With these limitations addressed and possible biomarkers mentioned, future experiments with this bioreactor will be conducted to study the mechanisms of TBI as the result of peak deceleration, impact duration, and biaxial strain. These experiments are only possible through the successful characterization 
of the bioreactor's parameters. The range of decelerations and strains are relatable to established experiments investigating the mechanical stimuli of TBI on various CNS-like and CNS cells. The future use of a CNS cell line and a component to greatly control the impact duration will better replicate the TBIlike scenario. By determining the dose sensitivity of astrocyte reactivity to mechanical trauma, a better understanding of injury severity may offer better diagnostic techniques that are based on the quantitative presence of this biomarker rather than the qualitative assessments that are used today. 
VII. FIGURES

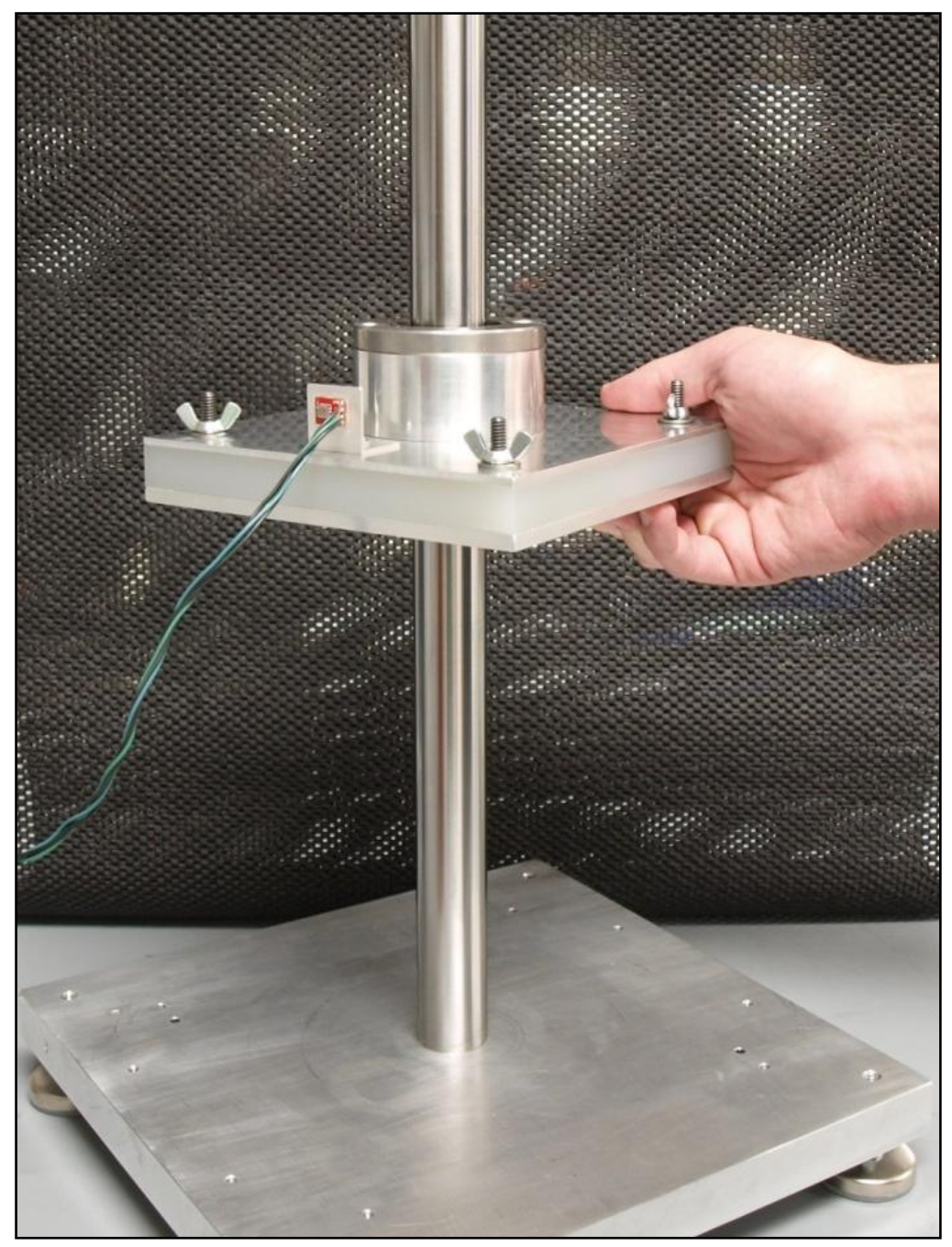

Figure 1: Assembled shuttle rose manually from the impact base and guided by a fixated rod. Mounted accelerometer is wired to a BUS-powered DAQ and PC not shown. The orientation of the accelerometer's single strain gage rosette is the same as the fixated rod: normal to the impact base. Photograph was taken by Zachery Heller. 
DAQmx Task Name

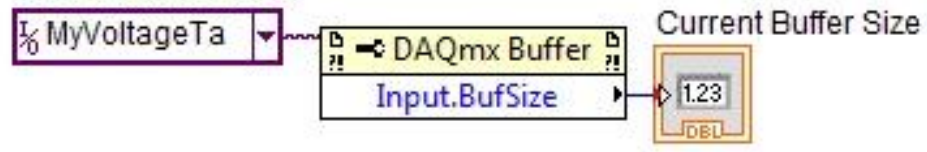

Record\&Save Triggered Signal

Waveform Chart

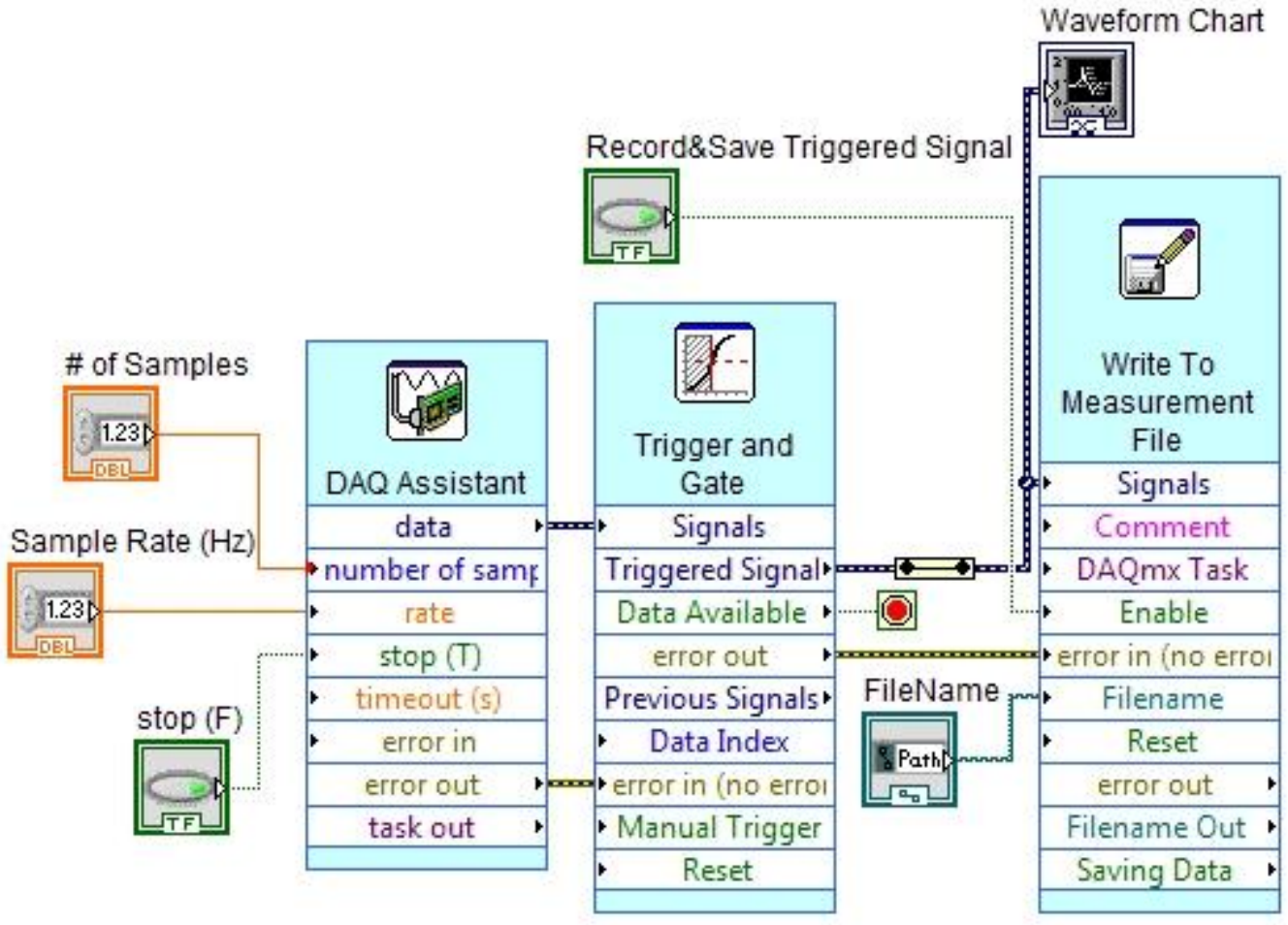

Erases Previous Chart History before Application start

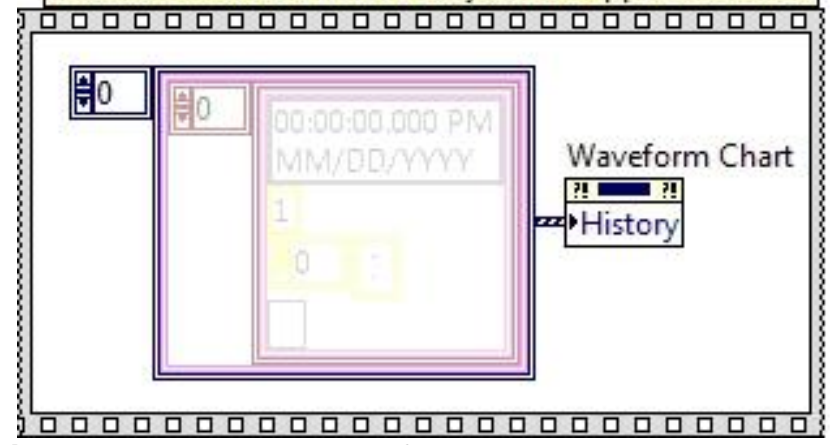

Figure 2: Block Diagram of LabVIEW program used to capture the impact profile. 


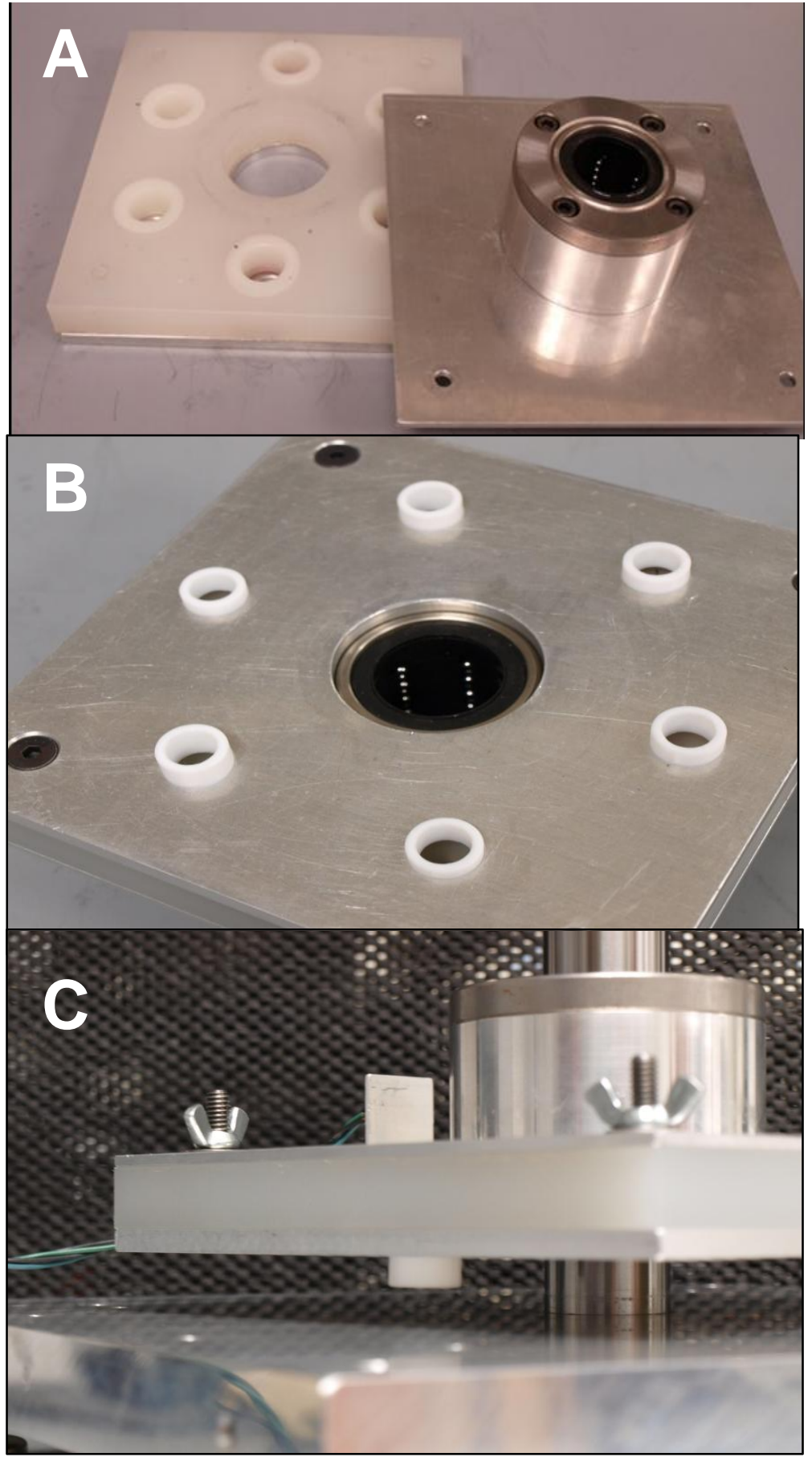

Figure 3: A) Disassembled shuttle with top plate removed and exposing implanted cell culture inserts within. B) Bottom view of shuttle with six teflon indenters placed in the holes of the bottom plate. C) Shuttle and indenter just before impact with impact base. Photographs were taken by Zachery Heller. 


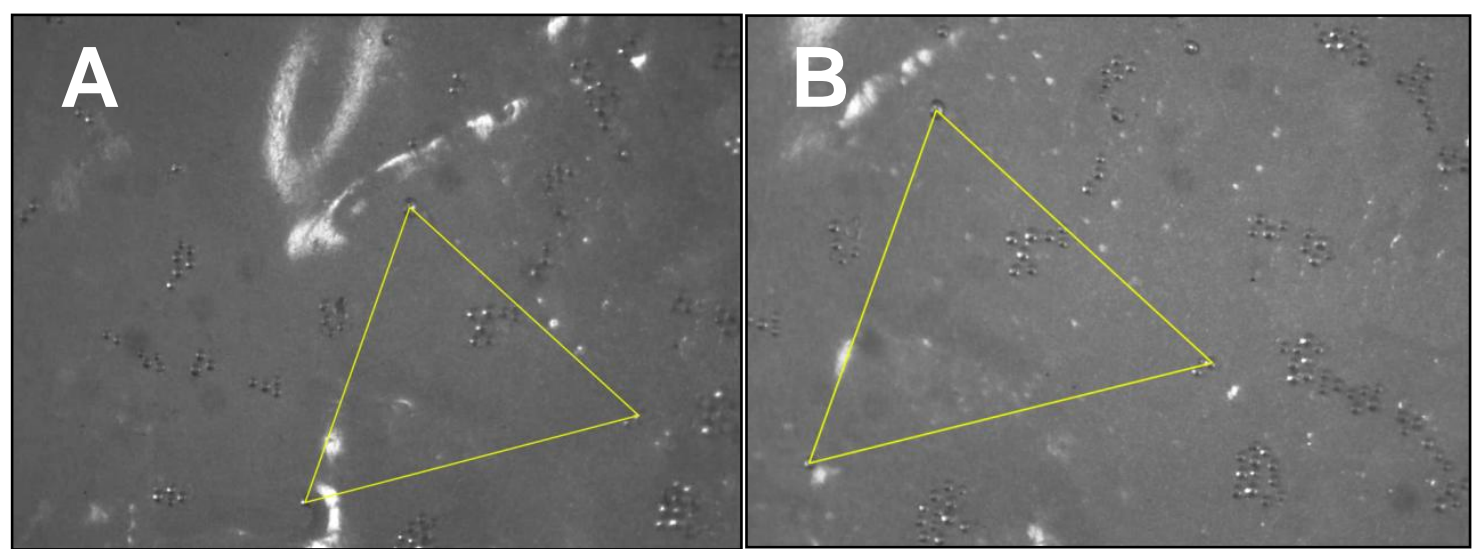

Figure 4: Grayscale images of membrane A) before and B) after indenting height of $5.57 \mathrm{~mm}$ ( 23\% Lagrangian strain). One specific triangular microbead arrangement is marked by the yellow triangle. Photographs were taken by Zachery Heller. 


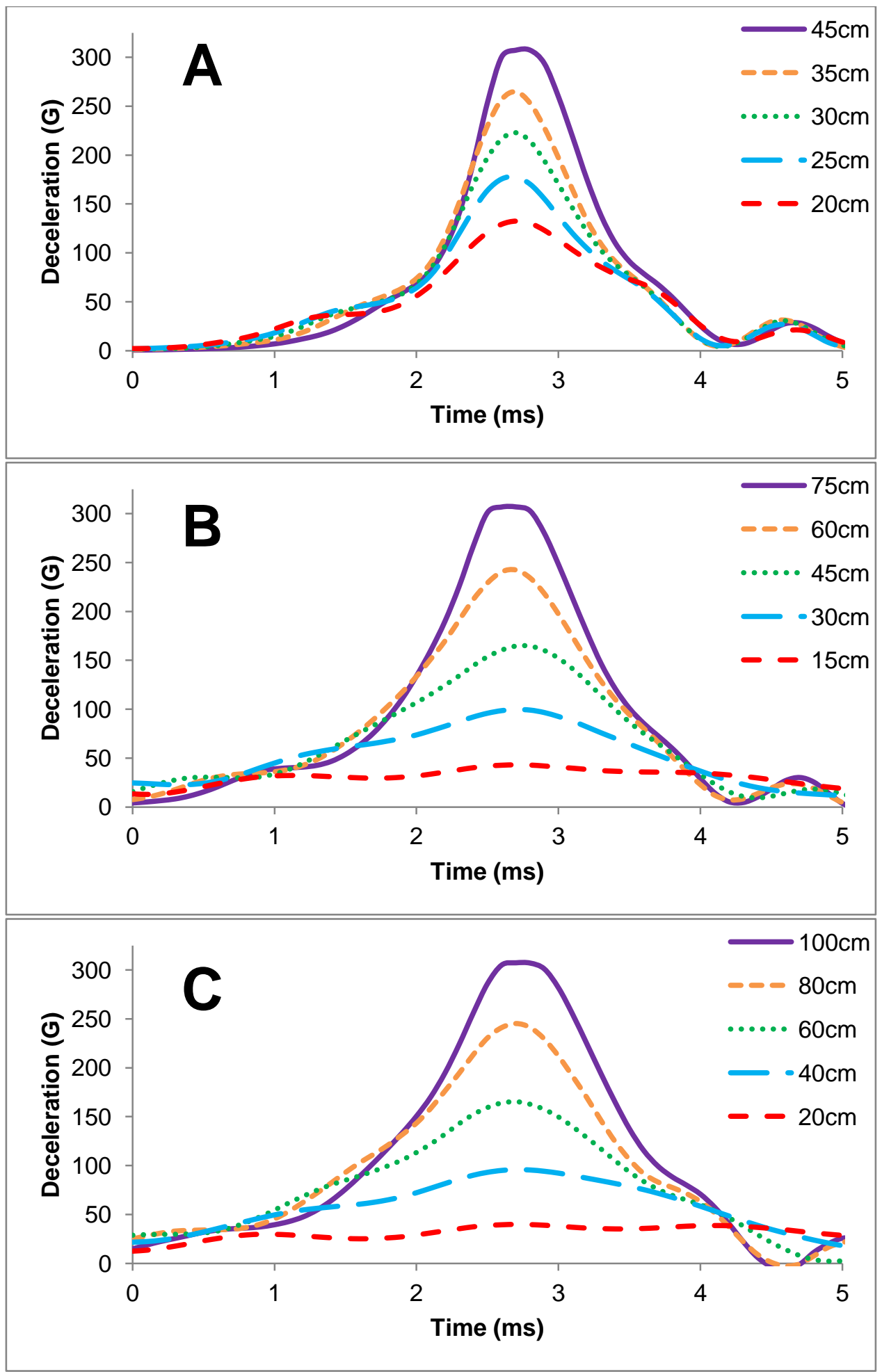

Figure 5: Mean impact profiles $(n=5)$ of the shuttle impacting the base with foam paddings of thicknesses A) $3.18 \mathrm{~mm}$, B) $6.35 \mathrm{~mm}$, and C) $9.53 \mathrm{~mm}$. The pooled standard deviation was A) $1.34 \times 10^{-2}$, B) $2.35 \times 10^{-2}$, and C) $1.43 \times 10^{-2}$. 


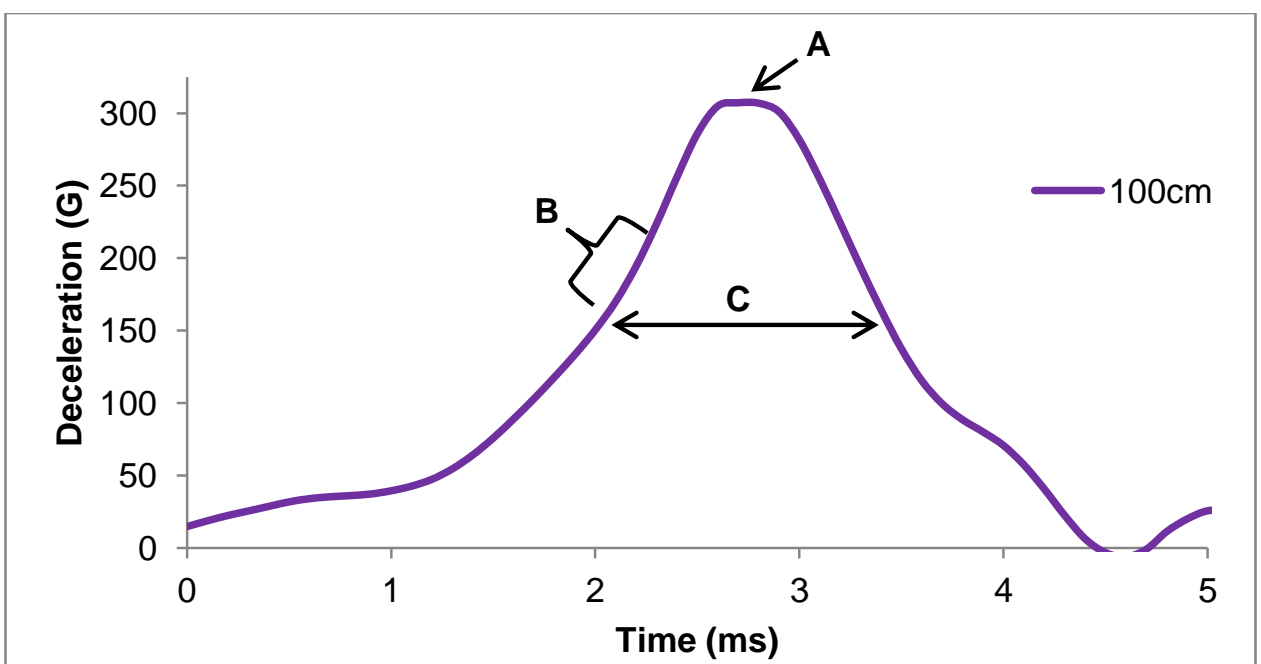

Figure 6: Impact profile of the shuttle impacting the base with a foam padding of $9.53 \mathrm{~mm}$-thickness at drop height. The characteristics of the impact profile noted were A) maximum deceleration, B) maximum jerk, and C) impact duration or halfpeak width. 


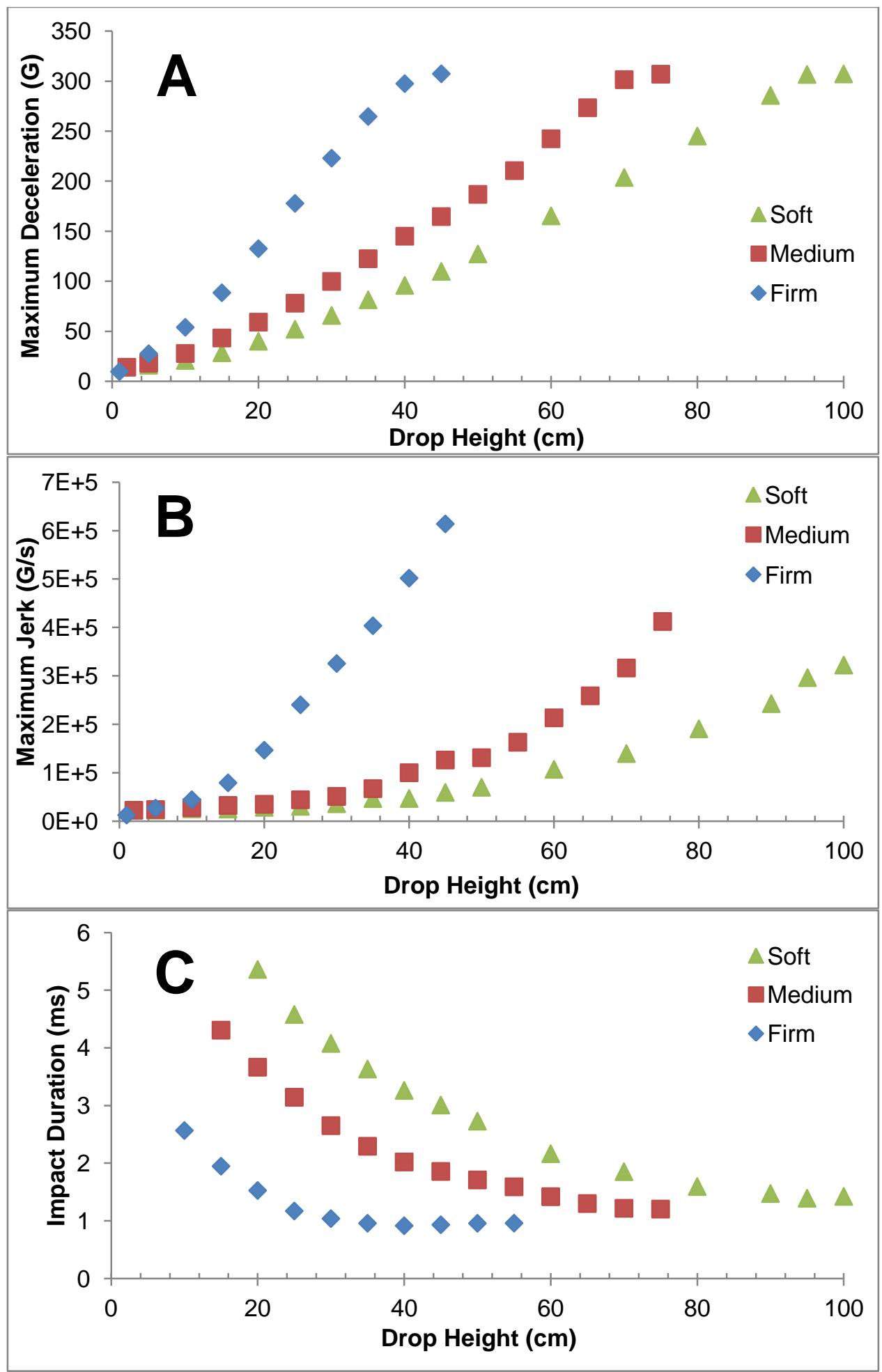

Figure 7: A)Mean maximum deceleration magnitude $(n=5)$ experienced by the shuttle when impacting the base with foam pad thicknesses of $3.18 \mathrm{~mm}$ (soft), $6.35 \mathrm{~mm}$ (medium), and $9.53 \mathrm{~mm}$ (firm). B) Maximum jerk derived from the mean impact profile. C) Half-peak width of impact profile of shuttle against the same foam pad thicknesses. 


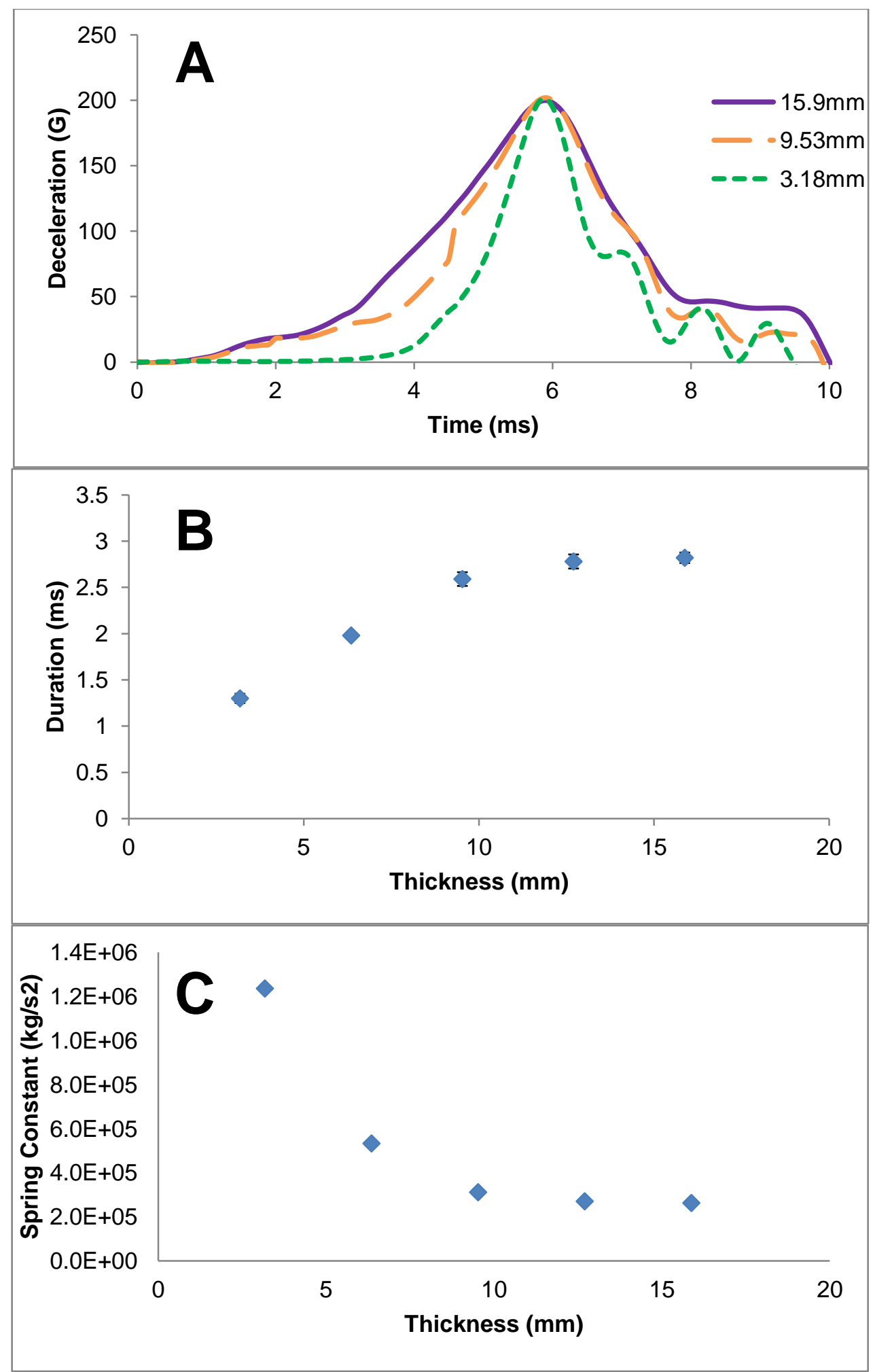

Figure 8: A) Mean impact profile $(n=5)$ of shuttle impact against specified thickness of pad. B) Mean impact duration of the impact profiles during shuttlepad impact. C) Calculated spring constant of pad thicknesses. 


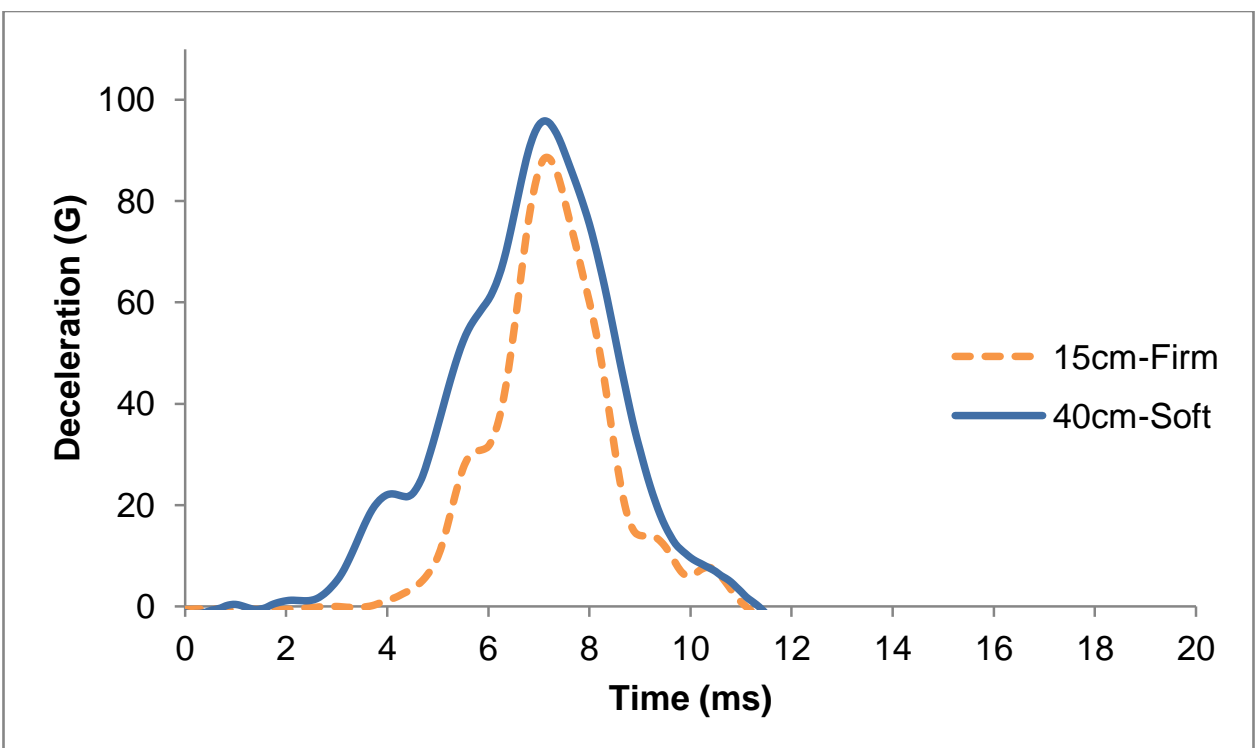

Figure 9: The impact profile when scaled to a larger timescale comparable to recorded $\sim 95 \mathrm{G}$ collisions using HITS. 


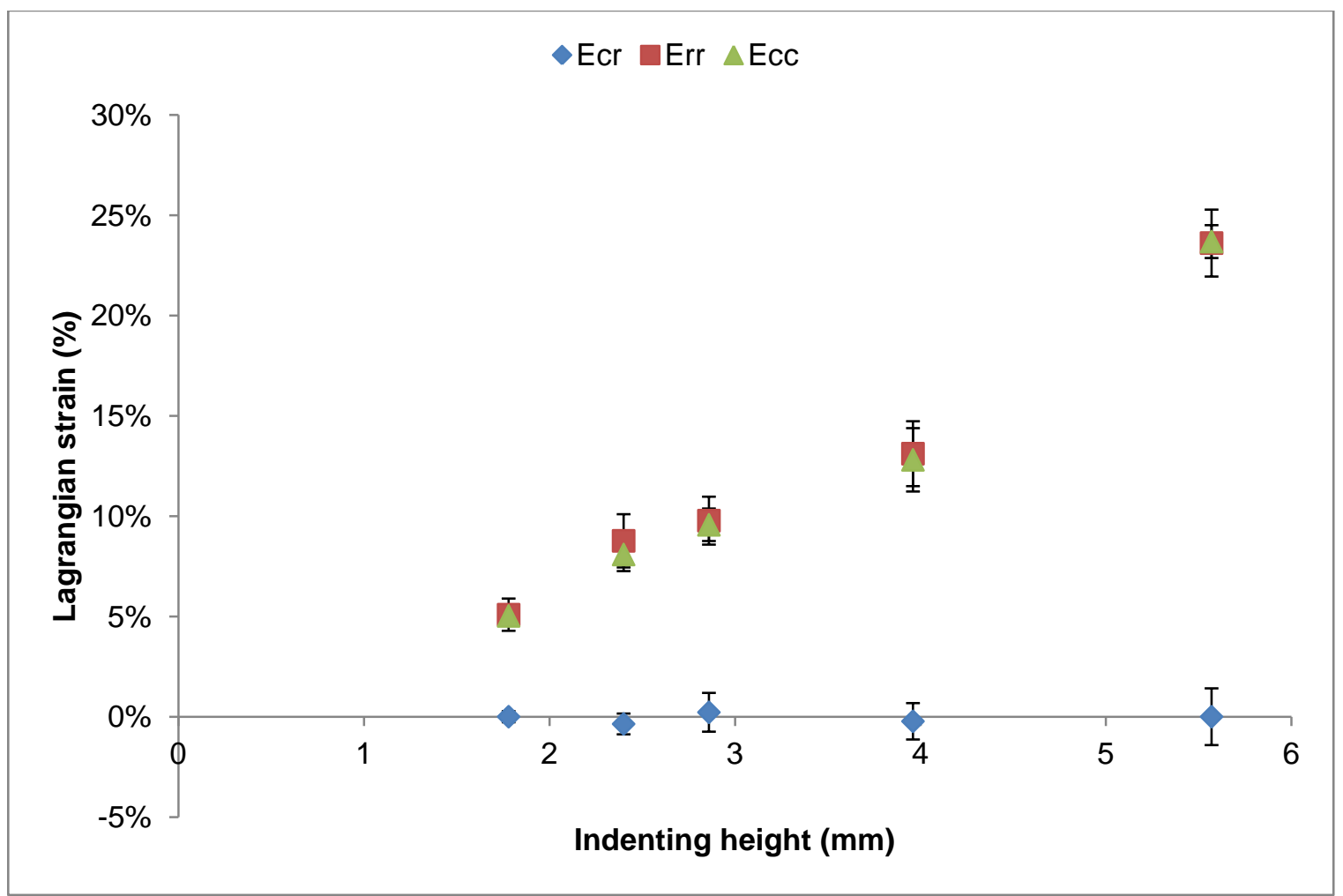

Figure 10: Mean principal Lagrangian strains $(n=9)$ of the membrane as a result of various indenting heights. 


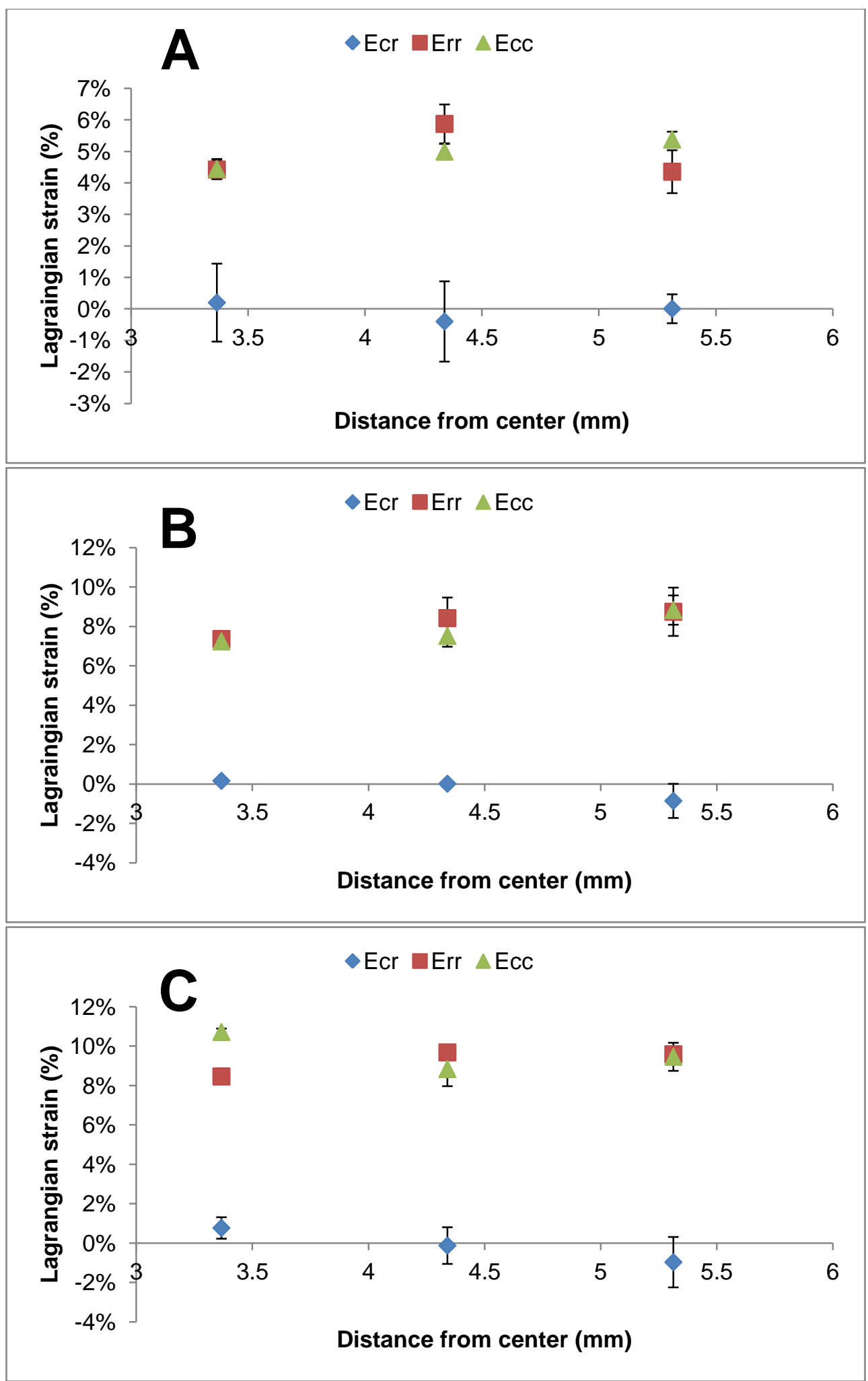

Figure 11: Mean principal Lagrangian strains $(n=3)$ recorded from various distances and produced by indenting heights of A) $1.78 \mathrm{~mm}$, B) $2.40 \mathrm{~mm}$, and C) $2.86 \mathrm{~mm}\left({ }^{*} \mathrm{p}<0.05\right.$ vs. control). 


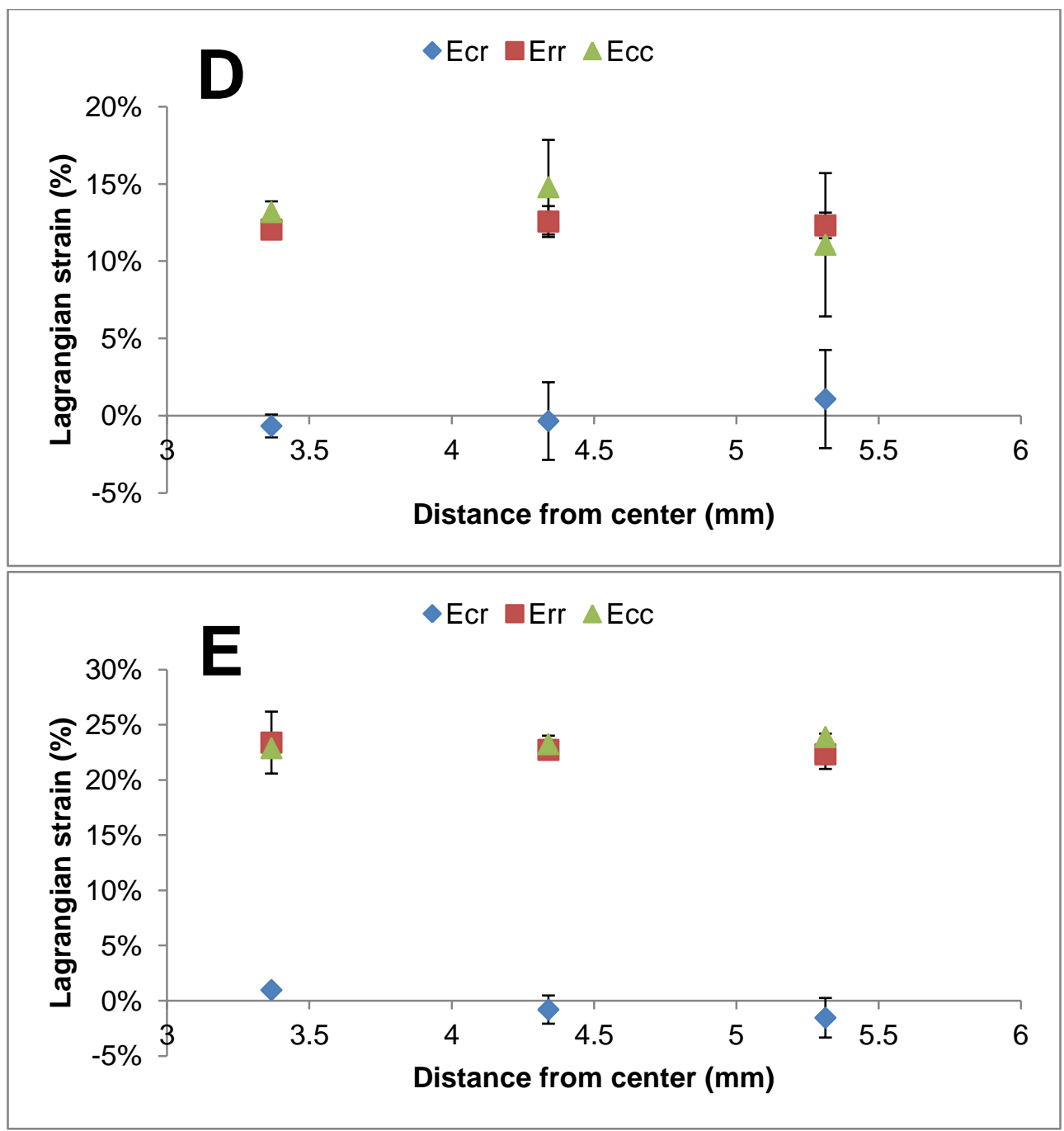

Figure 11: Mean principal Lagrangian strains $(n=3)$ from various distances and produced by indenting heights of D) $3.96 \mathrm{~mm}$ and E) $5.57 \mathrm{~mm} \mathrm{(}{ }^{*} p<0.05$ vs. control). 


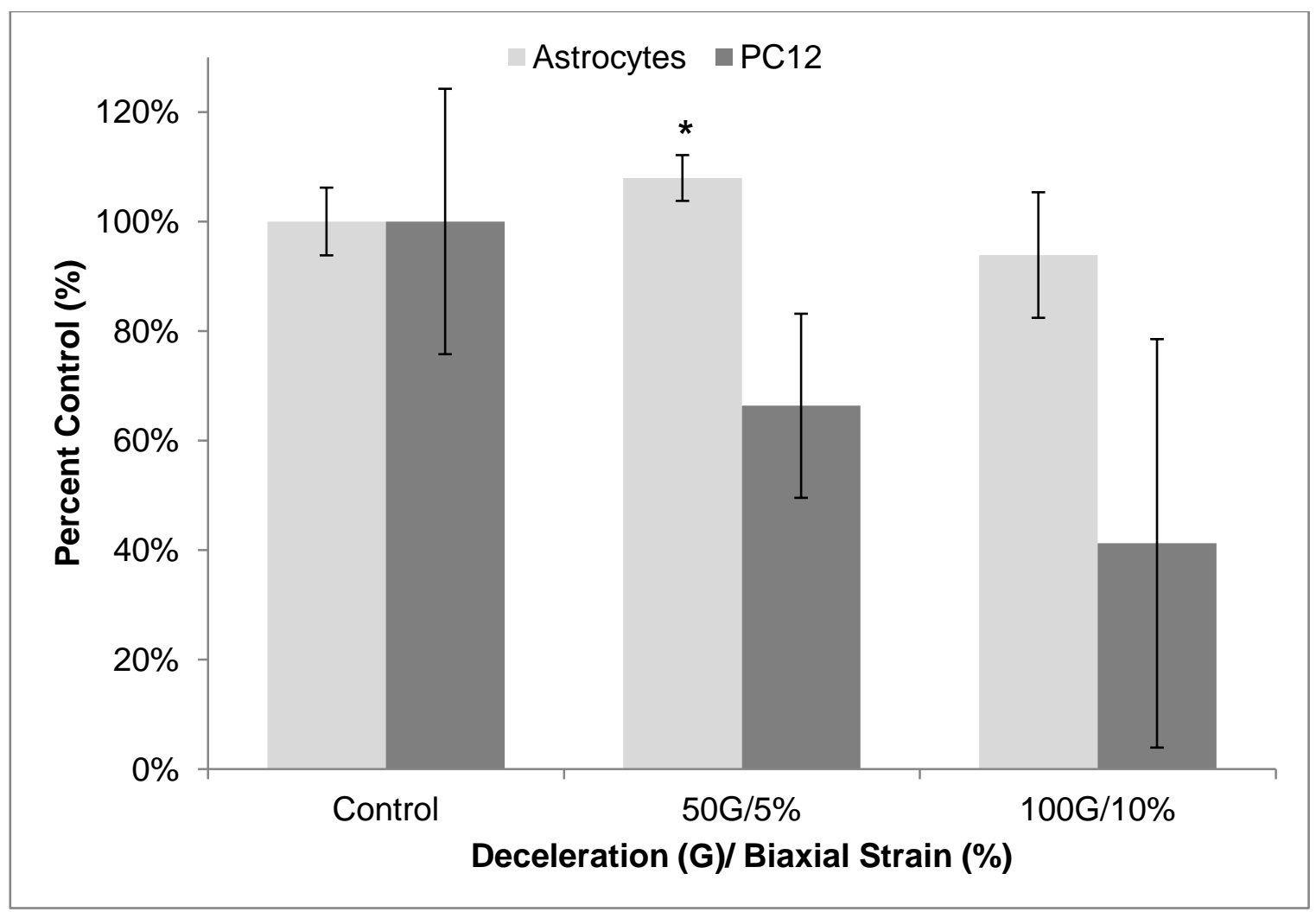

Figure 12: The average number of PC12 cells from captured fields $(n=10)$ and the resulting percent control when compared to the control. The SEM for each data set is displayed by the error bars $\left({ }^{*} p<0.05\right.$ vs. control). 


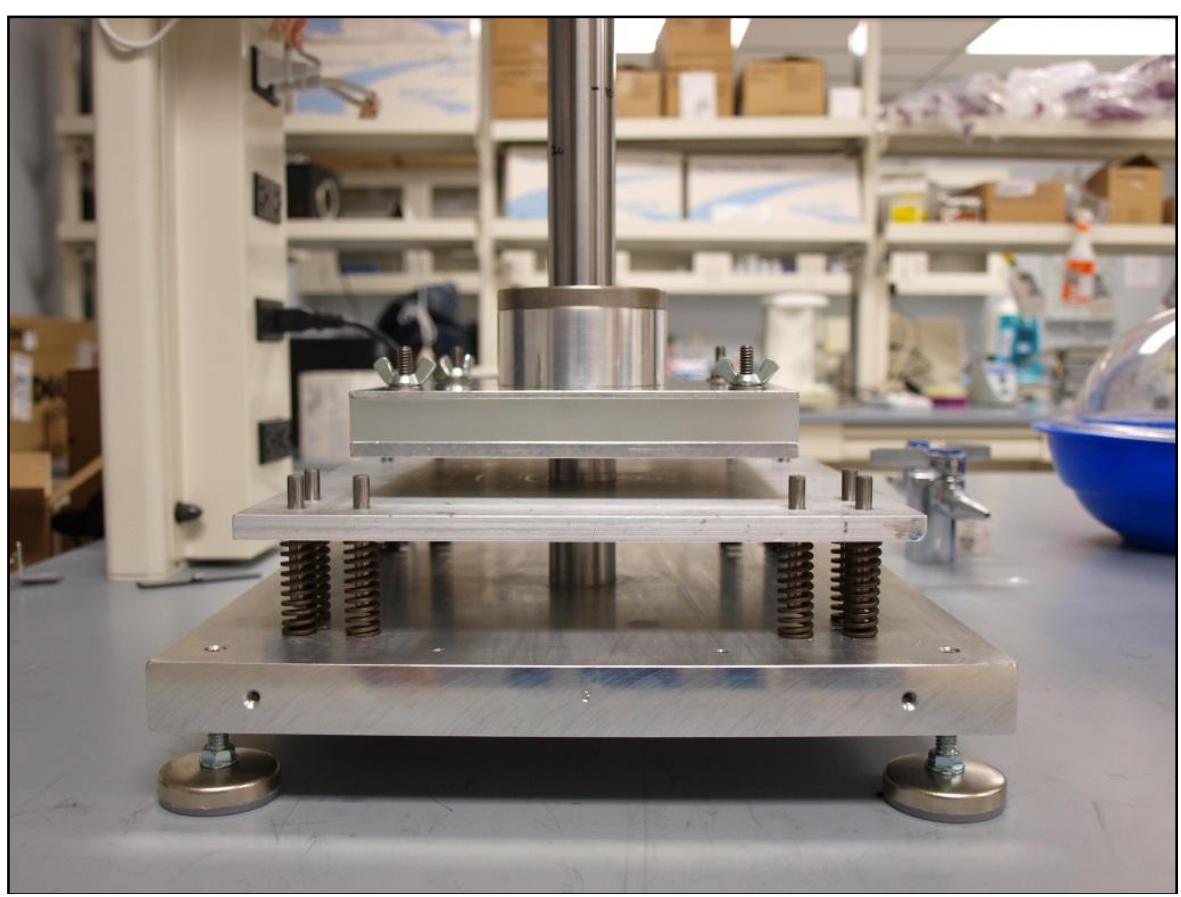

Figure 13: Preliminary design of additional component to bioreactor to control impact duration. Photograph was taken by Joseph Wyatt. 


\section{TABLES}

Table 1. Biomechanical data from HITS studies recording the number of impacts and head decelerations during impact from helmeted collisions.

\begin{tabular}{|l|c|c|c|c|}
\hline Authors \& Year & Athletic Level & No. of Impacts & $\begin{array}{c}\text { No. of } \\
\text { Concussions }\end{array}$ & $\begin{array}{c}\text { Mean Concussive } \\
\text { Impact (G) }\end{array}$ \\
\hline $\begin{array}{l}\text { Duma et al., } \\
2005^{39}\end{array}$ & college & 3,312 & 1 & 81 \\
\hline $\begin{array}{l}\text { Guskiewicz et al., } \\
2007^{58}\end{array}$ & college & 104,714 & 13 & 102.8 \\
\hline $\begin{array}{l}\text { Brolinson et al., } \\
2006^{20}\end{array}$ & college & 11,604 & 3 & 103.3 \\
\hline $\begin{array}{l}\text { Funk et al., } \\
2012^{47}\end{array}$ & college & 37,128 & 4 & 145 \\
\hline $\begin{array}{l}\text { Broglio et al., } \\
2010^{18}\end{array}$ & high school & 54,247 & 13 & 96.1 \\
\hline $\begin{array}{l}\text { Broglio et al., } \\
2011^{17}\end{array}$ & high school & 101,994 & 20 & 103.6 \\
\hline \multicolumn{1}{|c|}{ Average } & & & & 13.6 \\
\hline
\end{tabular}


Table 2. Parameters and regression from model-fitting curves of maximum deceleration and impact duration dependent on pad-thickness placed on the impact base. Models were determined from Microsoft Excel's Treadline tool; the model used for a specific curve is noted in parenthesis.

\begin{tabular}{|l|l|l|l|l|l|}
\hline $\begin{array}{l}\text { Thickness } \\
(\mathbf{m m})\end{array}$ & $\begin{array}{l}\text { Max } \\
\text { Deceleration } \\
\text { (linear-slope) }\end{array}$ & Regression & $\begin{array}{l}\text { Impact } \\
\text { Duration } \\
\text { (power- } \\
\text { coefficient) }\end{array}$ & $\begin{array}{l}\text { Impact Duration } \\
\text { (power- } \\
\text { exponent) }\end{array}$ & Regression \\
\hline 3.18 & $7.25 \mathrm{x}$ & 0.986 & 9.86 & -0.623 & 0.912 \\
\hline 6.38 & $3.90 \mathrm{x}$ & 0.975 & 44.71 & -0.839 & 0.994 \\
\hline 9.53 & $2.92 \mathrm{x}$ & 0.963 & 82.61 & -0.888 & 0.991 \\
\hline
\end{tabular}




\section{REFERENCES}

1. Aloisi, F. (2001). Immune function of microglia. Glia, 36(2), 165-179.

2. Arnadottir, J., \& Chalfie, M. (2010). Eukaryotic mechanosensitive channels. Annual Review of Biophysics, 39, 111-137. doi:10.1146/annurev.biophys.37.032807.125836; 10.1146/annurev.biophys.37.032807.125836

3. Bain, A. C., \& Meaney, D. F. (2000). Tissue-level thresholds for axonal damage in an experimental model of central nervous system white matter injury. Journal of Biomechanical Engineering, 122(6), 615-622.

4. Baldwin, S. A., Fugaccia, I., Brown, D. R., Brown, L. V., \& Scheff, S. W. (1996). Blood-brain barrier breach following cortical contusion in the rat. Journal of Neurosurgery, 85(3), 476-481. doi:10.3171/jns.1996.85.3.0476

5. Barkhoudarian, G., Hovda, D. A., \& Giza, C. C. (2011). The molecular pathophysiology of concussive brain injury. Clinics in Sports Medicine, 30(1), 33-48. doi:10.1016/j.csm.2010.09.001

6. Barley, K., Dracheva, S., \& Byne, W. (2009). Subcortical oligodendrocyte- and astrocyte-associated gene expression in subjects with schizophrenia, major depression and bipolar disorder.

Schizophrenia Research, 112(1-3), 54-64. doi:10.1016/j.schres.2009.04.019; 10.1016/j.schres.2009.04.019

7. Bayly, P. V., Black, E. E., Pedersen, R. C., Leister, E. P., \& Genin, G. M. (2006). In vivo imaging of rapid deformation and strain in an animal model of traumatic brain injury. Journal of Biomechanics, 39(6), 1086-1095. doi:10.1016/j.jbiomech.2005.02.014

8. Bayly, P. V., Cohen, T. S., Leister, E. P., Ajo, D., Leuthardt, E. C., \& Genin, G. M. (2005). Deformation of the human brain induced by mild acceleration. Journal of Neurotrauma, 22(8), 845-856. doi:10.1089/neu.2005.22.845

9. Bazarian, J. J. (2010). Diagnosing mild traumatic brain injury after a concussion. The Journal of Head Trauma Rehabilitation, 25(4), 225-227. doi:10.1097/HTR.0b013e3181e7f784; 10.1097/HTR.0b013e3181e7f784

10. Bazarian, J. J., Blyth, B. J., He, H., Mookerjee, S., Jones, C., Kiechle, K., . . Khan, J. (2013). Classification accuracy of serum apo A-I and S100B for the diagnosis of mild traumatic brain injury and prediction of abnormal initial head computed tomography scan. Journal of Neurotrauma, 30(20), 1747-1754. doi:10.1089/neu.2013.2853; 10.1089/neu.2013.2853

11. Belanger, H. G., Vanderploeg, R. D., Curtiss, G., \& Warden, D. L. (2007). Recent neuroimaging techniques in mild traumatic brain injury. The Journal of Neuropsychiatry and Clinical Neurosciences, 19(1), 5-20. doi:10.1176/appi.neuropsych.19.1.5

12. Bey, T., \& Ostick, B. (2009). Second impact syndrome. The Western Journal of Emergency Medicine, 10(1), 6-10.

13. Blaylock, R. L., \& Maroon, J. (2011). Immunoexcitotoxicity as a central mechanism in chronic traumatic encephalopathy-A unifying hypothesis. Surgical Neurology International, 2, 1077806.83391. Epub 2011 Jul 30. doi:10.4103/2152-7806.83391; 10.4103/2152-7806.83391 
14. Blyth, B. J., Nayak, A., Sparks, J. D., \& Khan, J. (2010). Apolipoprotein A1 is increased in the serum of mild traumatic brain injury patients. Journal of Neurotrauma, 26, 25.

15. Bowen, A. P. (2003). Second impact syndrome: A rare, catastrophic, preventable complication of concussion in young athletes. Journal of Emergency Nursing: JEN : Official Publication of the Emergency Department Nurses Association, 29(3), 287-289.

16. Broglio, S. P., Eckner, J. T., Martini, D., Sosnoff, J. J., Kutcher, J. S., \& Randolph, C. (2011). Cumulative head impact burden in high school football. Journal of Neurotrauma, 28(10), 2069-2078. doi:10.1089/neu.2011.1825; 10.1089/neu.2011.1825

17. Broglio, S. P., Eckner, J. T., Surma, T., \& Kutcher, J. S. (2011). Post-concussion cognitive declines and symptomatology are not related to concussion biomechanics in high school football players. Journal of Neurotrauma, 28(10), 2061-2068. doi:10.1089/neu.2011.1905; 10.1089/neu.2011.1905

18. Broglio, S. P., Schnebel, B., Sosnoff, J. J., Shin, S., Fend, X., He, X., \& Zimmerman, J. (2010). Biomechanical properties of concussions in high school football. Medicine and Science in Sports and Exercise, 42(11), 2064-2071. doi:10.1249/MSS.0b013e3181dd9156; 10.1249/MSS.0b013e3181dd9156

19. Broglio, S. P., Sosnoff, J. J., Shin, S., He, X., Alcaraz, C., \& Zimmerman, J. (2009). Head impacts during high school football: A biomechanical assessment. Journal of Athletic Training, 44(4), 342349. doi:10.4085/1062-6050-44.4.342; 10.4085/1062-6050-44.4.342

20. Brolinson, P. G., Manoogian, S., McNeely, D., Goforth, M., Greenwald, R., \& Duma, S. (2006). Analysis of linear head accelerations from collegiate football impacts. Current Sports Medicine Reports, 5(1), 23-28.

21. Brown, H. G., \& Hoh, J. H. (1997). Entropic exclusion by neurofilament sidearms: A mechanism for maintaining interfilament spacing. Biochemistry, 36(49), 15035-15040. doi:10.1021/bi9721748

22. Bushong, E. A., Martone, M. E., Jones, Y. Z., \& Ellisman, M. H. (2002). Protoplasmic astrocytes in CA1 stratum radiatum occupy separate anatomical domains. The Journal of Neuroscience : The Official Journal of the Society for Neuroscience, 22(1), 183-192.

23. Cahoy, J. D., Emery, B., Kaushal, A., Foo, L. C., Zamanian, J. L., Christopherson, K. S., . . Barres, B. A. (2008). A transcriptome database for astrocytes, neurons, and oligodendrocytes: A new resource for understanding brain development and function. The Journal of Neuroscience : The Official Journal of the Society for Neuroscience, 28(1), 264-278. doi:10.1523/JNEUROSCI.417807.2008; 10.1523/JNEUROSCI.4178-07.2008

24. Cantu, R. C. (1998). Second-impact syndrome. Clinics in Sports Medicine, 17(1), 37-44.

25. Cater, H. L., Sundstrom, L. E., \& Morrison, B.,3rd. (2006). Temporal development of hippocampal cell death is dependent on tissue strain but not strain rate. Journal of Biomechanics, 39(15), 2810-2818. doi:10.1016/j.jbiomech.2005.09.023

26. Cazaubon, S., Parker, P. J., Strosberg, A. D., \& Couraud, P. O. (1993). Endothelins stimulate tyrosine phosphorylation and activity of p42/mitogen-activated protein kinase in astrocytes. The Biochemical Journal, 293 ( Pt 2)(Pt 2), 381-386.

27. Chavis, P., \& Westbrook, G. (2001). Integrins mediate functional pre- and postsynaptic maturation at a hippocampal synapse. Nature, 411(6835), 317-321. doi:10.1038/35077101 
28. Cherian, L., Robertson, C. S., Contant, C. F.,Jr, \& Bryan, R. M.,Jr. (1994). Lateral cortical impact injury in rats: Cerebrovascular effects of varying depth of cortical deformation and impact velocity. Journal of Neurotrauma, 11(5), 573-585.

29. Coats, B., \& Margulies, S. S. (2006). Material properties of porcine parietal cortex. Journal of Biomechanics, 39(13), 2521-2525. doi:10.1016/j.jbiomech.2005.07.020

30. Collins, M. W., Iverson, G. L., Lovell, M. R., McKeag, D. B., Norwig, J., \& Maroon, J. (2003). On-field predictors of neuropsychological and symptom deficit following sports-related concussion. Clinical Journal of Sport Medicine : Official Journal of the Canadian Academy of Sport Medicine, 13(4), 222229.

31. Coronado, V. G., Xu, L., Basavaraju, S. V., McGuire, L. C., Wald, M. M., Faul, M. D., . . Centers for Disease Control and Prevention (CDC). (2011). Surveillance for traumatic brain injury-related deaths--united states, 1997-2007. Morbidity and Mortality Weekly Report.Surveillance Summaries (Washington, D.C.: 2002), 60(5), 1-32.

32. Crawford, G. E., \& Earnshaw, J. C. (1987). Viscoelastic relaxation of bilayer lipid membranes. frequency-dependent tension and membrane viscosity. Biophysical Journal, 52(1), 87-94. doi:10.1016/S0006-3495(87)83191-0

33. Crisco, J. J., Fiore, R., Beckwith, J. G., Chu, J. J., Brolinson, P. G., Duma, S., . . Greenwald, R. M. (2010). Frequency and location of head impact exposures in individual collegiate football players. Journal of Athletic Training, 45(6), 549-559. doi:10.4085/1062-6050-45.6.549; 10.4085/1062-605045.6.549

34. Daniel, R. W., Rowson, S., \& Duma, S. M. (2012). Head impact exposure in youth football. Annals of Biomedical Engineering, 40(4), 976-981. doi:10.1007/s10439-012-0530-7; 10.1007/s10439-0120530-7

35. Di, X., Goforth, P. B., Bullock, R., Ellis, E., \& Satin, L. (2000). Mechanical injury alters volume activated ion channels in cortical astrocytes. Acta Neurochirurgica.Supplement, 76, 379-383.

36. Dityatev, A., Schachner, M., \& Sonderegger, P. (2010). The dual role of the extracellular matrix in synaptic plasticity and homeostasis. Nature Reviews.Neuroscience, 11(11), 735-746. doi:10.1038/nrn2898; 10.1038/nrn2898

37. Dixon, C. E., Clifton, G. L., Lighthall, J. W., Yaghmai, A. A., \& Hayes, R. L. (1991). A controlled cortical impact model of traumatic brain injury in the rat. Journal of Neuroscience Methods, 39(3), 253-262.

38. Donnelly, B. R., \& Medige, J. (1997). Shear properties of human brain tissue. Journal of Biomechanical Engineering, 119(4), 423-432.

39. Duma, S. M., Manoogian, S. J., Bussone, W. R., Brolinson, P. G., Goforth, M. W., Donnenwerth, J. J., ... Crisco, J. J. (2005). Analysis of real-time head accelerations in collegiate football players. Clinical Journal of Sport Medicine : Official Journal of the Canadian Academy of Sport Medicine, 15(1), 3-8.

40. Ehrenreich, H., Anderson, R. W., Ogino, Y., Rieckmann, P., Costa, T., Wood, G. P., . . Fauci, A. S. (1991). Selective autoregulation of endothelins in primary astrocyte cultures: Endothelin receptormediated potentiation of endothelin-1 secretion. The New Biologist, 3(2), 135-141. 
41. Ehrenreich, H., Kehrl, J. H., Anderson, R. W., Rieckmann, P., Vitkovic, L., Coligan, J. E., \& Fauci, A. S. (1991). A vasoactive peptide, endothelin-3, is produced by and specifically binds to primary astrocytes. Brain Research, 538(1), 54-58.

42. Elkin, B. S., Azeloglu, E. U., Costa, K. D., \& Morrison, B.,3rd. (2007). Mechanical heterogeneity of the rat hippocampus measured by atomic force microscope indentation. Journal of Neurotrauma, 24(5), 812-822. doi:10.1089/neu.2006.0169

43. Ellis, E. F., McKinney, J. S., Willoughby, K. A., Liang, S., \& Povlishock, J. T. (1995). A new model for rapid stretch-induced injury of cells in culture: Characterization of the model using astrocytes.

Journal of Neurotrauma, 12(3), 325-339.

44. Epstein, M. H. (1971). Relative susceptibility of elements of the cerebral cortex to mechanical trauma in the rat. Journal of Neurosurgery, 35(5), 517-522. doi:10.3171/jns.1971.35.5.0517

45. Finkelstein, E., Corso, P., \& Miller, T. (2006). The incidence and economic burden of injuries in the united states. New York: Oxford University Press.

46. Funk, J. R., Duma, S. M., Manoogian, S. J., \& Rowson, S. (2007). Biomechanical risk estimates for mild traumatic brain injury. Annual Proceedings / Association for the Advancement of Automotive Medicine.Association for the Advancement of Automotive Medicine, 51, 343-361.

47. Funk, J. R., Rowson, S., Daniel, R. W., \& Duma, S. M. (2012). Validation of concussion risk curves for collegiate football players derived from HITS data. Annals of Biomedical Engineering, 40(1), 79-89. doi:10.1007/s10439-011-0400-8; 10.1007/s10439-011-0400-8

48. Galkin, V. E., Orlova, A., \& Egelman, E. H. (2012). Actin filaments as tension sensors. Current Biology :CB, 22(3), R96-101. doi:10.1016/j.cub.2011.12.010; 10.1016/j.cub.2011.12.010

49. Geddes, D. M., \& Cargill, R. S.,2nd. (2001). An in vitro model of neural trauma: Device characterization and calcium response to mechanical stretch. Journal of Biomechanical Engineering, 123(3), 247-255.

50. Geddes, D. M., Cargill, R. S.,2nd, \& LaPlaca, M. C. (2003). Mechanical stretch to neurons results in a strain rate and magnitude-dependent increase in plasma membrane permeability. Journal of Neurotrauma, 20(10), 1039-1049. doi:10.1089/089771503770195885

51. Gefen, A., Gefen, N., Zhu, Q., Raghupathi, R., \& Margulies, S. S. (2003). Age-dependent changes in material properties of the brain and braincase of the rat. Journal of Neurotrauma, 20(11), 1163-1177. doi:10.1089/089771503770802853

52. Gennarelli, T. A. (1993). Mechanisms of brain injury. The Journal of Emergency Medicine, 11, 5-11.

53. Gilchrist, M. D. (2004). Experimental device for simulating traumatic brain injury resulting from linear accelerations. Strain, 40(4), 180-192. doi:10.1111/j.1475-1305.2004.00168.x

54. Gourine, A. V., Kasymov, V., Marina, N., Tang, F., Figueiredo, M. F., Lane, S., . . Kasparov, S. (2010). Astrocytes control breathing through pH-dependent release of ATP. Science (New York, N.Y.), 329(5991), 571-575. doi:10.1126/science.1190721; 10.1126/science.1190721 
55. Greene, L. A., Aletta, J. M., Rukenstein, A., \& Green, S. H. (1987). PC12 pheochromocytoma cells: Culture, nerve growth factor treatment, and experimental exploitation. Methods in Enzymology, 147, 207-216.

56. Greene, L. A., \& Tischler, A. S. (1976). Establishment of a noradrenergic clonal line of rat adrenal pheochromocytoma cells which respond to nerve growth factor. Proceedings of the National Academy of Sciences of the United States of America, 73(7), 2424-2428.

57. GURDJIAN, E. S., LISSNER, H. R., EVANS, F. G., PATRICK, L. M., \& HARDY, W. G. (1961). Intracranial pressure and acceleration accompanying head impacts in human cadavers. Surgery, Gynecology \& Obstetrics, 113, 185-190.

58. Guskiewicz, K. M., Mihalik, J. P., Shankar, V., Marshall, S. W., Crowell, D. H., Oliaro, S. M., .. . Hooker, D. N. (2007). Measurement of head impacts in collegiate football players: Relationship between head impact biomechanics and acute clinical outcome after concussion. Neurosurgery, 61(6), 1244-52; discussion 1252-3. doi:10.1227/01.neu.0000306103.68635.1a

59. Hama, H., Kasuya, Y., Sakurai, T., Yamada, G., Suzuki, N., Masaki, T., \& Goto, K. (1997). Role of endothelin-1 in astrocyte responses after acute brain damage. Journal of Neuroscience Research, $47(6), 590-602$.

60. Hamill, O. P., \& Martinac, B. (2001). Molecular basis of mechanotransduction in living cells. Physiological Reviews, 81(2), 685-740.

61. Hardy, W. N., Mason, M. J., Foster, C. D., Shah, C. S., Kopacz, J. M., Yang, K. H., . . Tashman, S. (2007). A study of the response of the human cadaver head to impact. Stapp Car Crash Journal, 51, 17-80.

62. Hemphill, M. A., Dabiri, B. E., Gabriele, S., Kerscher, L., Franck, C., Goss, J. A., . . Parker, K. K. (2011). A possible role for integrin signaling in diffuse axonal injury. PloS One, 6(7), e22899.

doi:10.1371/journal.pone.0022899; 10.1371/journal.pone.0022899

63. Hirtz, D., Thurman, D. J., Gwinn-Hardy, K., Mohamed, M., Chaudhuri, A. R., \& Zalutsky, R. (2007). How common are the "common" neurologic disorders? Neurology, 68(5), 326-337. doi:10.1212/01.wnl.0000252807.38124.a3

64. Hoffman, S. W., Rzigalinski, B. A., Willoughby, K. A., \& Ellis, E. F. (2000). Astrocytes generate isoprostanes in response to trauma or oxygen radicals. Journal of Neurotrauma, 17(5), 415-420.

65. Holbourn, A. H. S. (1943). The mechanics of head injury. Lancet, , 438-441.

66. Hu, X., Viesselmann, C., Nam, S., Merriam, E., \& Dent, E. W. (2008). Activity-dependent dynamic microtubule invasion of dendritic spines. The Journal of Neuroscience : The Official Journal of the Society for Neuroscience, 28(49), 13094-13105. doi:10.1523/JNEUROSCI.3074-08.2008; 10.1523/JNEUROSCI.3074-08.2008

67. Ivancevic, V. G. (2009). New mechanics of traumatic brain injury. Cognitive Neurodynamics, 3(3), 281-293. doi:10.1007/s11571-008-9070-0; 10.1007/s11571-008-9070-0

68. Jaworski, J., Kapitein, L. C., Gouveia, S. M., Dortland, B. R., Wulf, P. S., Grigoriev, I., . . Hoogenraad, C. C. (2009). Dynamic microtubules regulate dendritic spine morphology and synaptic plasticity. Neuron, 61(1), 85-100. doi:10.1016/j.neuron.2008.11.013; 10.1016/j.neuron.2008.11.013 
69. Kasuya, Y., Abe, Y., Hama, H., Sakurai, T., Asada, S., Masaki, T., \& Goto, K. (1994). Endothelin-1 activates mitogen-activated protein kinases through two independent signalling pathways in rat astrocytes. Biochemical and Biophysical Research Communications, 204(3), 1325-1333.

70. Kawamata, T., Katayama, Y., Hovda, D. A., Yoshino, A., \& Becker, D. P. (1995). Lactate accumulation following concussive brain injury: The role of ionic fluxes induced by excitatory amino acids. Brain Research, 674(2), 196-204.

71. Kruse, S. A., Rose, G. H., Glaser, K. J., Manduca, A., Felmlee, J. P., Jack, C. R.,Jr, \& Ehman, R. L. (2008). Magnetic resonance elastography of the brain. Neurolmage, 39(1), 231-237. doi:10.1016/j.neuroimage.2007.08.030

72. Kueh, H. Y., \& Mitchison, T. J. (2009). Structural plasticity in actin and tubulin polymer dynamics. Science (New York, N.Y.), 325(5943), 960-963. doi:10.1126/science.1168823; 10.1126/science. 1168823

73. Kumaria, A., \& Tolias, C. M. (2008). In vitro models of neurotrauma. British Journal of Neurosurgery, 22(2), 200-206. doi:10.1080/02688690701772413; 10.1080/02688690701772413

74. Lamb, R. G., Harper, C. C., McKinney, J. S., Rzigalinski, B. A., \& Ellis, E. F. (1997). Alterations in phosphatidylcholine metabolism of stretch-injured cultured rat astrocytes. Journal of Neurochemistry, 68(5), 1904-1910.

75. Langlois, J. A., Rutland-Brown, W., \& Wald, M. M. (2006). The epidemiology and impact of traumatic brain injury: A brief overview. The Journal of Head Trauma Rehabilitation, 21(5), 375-378.

76. Lazarini, F., Strosberg, A. D., Couraud, P. O., \& Cazaubon, S. M. (1996). Coupling of ETB endothelin receptor to mitogen-activated protein kinase stimulation and DNA synthesis in primary cultures of rat astrocytes. Journal of Neurochemistry, 66(2), 459-465.

77. Lee, A. A., Delhaas, T., Waldman, L. K., MacKenna, D. A., Villarreal, F. J., \& McCulloch, A. D. (1996). An equibiaxial strain system for cultured cells. The American Journal of Physiology, 271(4 Pt 1), C1400-8.

78. Lee, M. K., \& Cleveland, D. W. (1996). Neuronal intermediate filaments. Annual Review of Neuroscience, 19, 187-217. doi:10.1146/annurev.ne.19.030196.001155

79. Lighthall, J. W. (1988). Controlled cortical impact: A new experimental brain injury model. Journal of Neurotrauma, 5(1), 1-15.

80. Lo, E. H., Wang, X., \& Cuzner, M. L. (2002). Extracellular proteolysis in brain injury and inflammation: Role for plasminogen activators and matrix metalloproteinases. Journal of Neuroscience Research, 69(1), 1-9. doi:10.1002/jnr.10270

81. Lo, F. S., Zhao, S., \& Erzurumlu, R. S. (2011). Astrocytes promote peripheral nerve injury-induced reactive synaptogenesis in the neonatal CNS. Journal of Neurophysiology, 106(6), 2876-2887. doi:10.1152/jn.00312.2011; 10.1152/jn.00312.2011

82. Longhi, L., Saatman, K. E., Fujimoto, S., Raghupathi, R., Meaney, D. F., Davis, J., . . Mclntosh, T. K. (2005). Temporal window of vulnerability to repetitive experimental concussive brain injury. Neurosurgery, 56(2), 364-74; discussion 364-74. 
83. Loov, C., Hillered, L., Ebendal, T., \& Erlandsson, A. (2012). Engulfing astrocytes protect neurons from contact-induced apoptosis following injury. PloS One, 7(3), e33090.

doi:10.1371/journal.pone.0033090; 10.1371/journal.pone.0033090

84. Loov, C., Shevchenko, G., Geeyarpuram Nadadhur, A., Clausen, F., Hillered, L., Wetterhall, M., \& Erlandsson, A. (2013). Identification of injury specific proteins in a cell culture model of traumatic brain injury. PloS One, 8(2), e55983. doi:10.1371/journal.pone.0055983; 10.1371/journal.pone.0055983

85. Lucas, J. H., \& Wolf, A. (1991). In vitro studies of multiple impact injury to mammalian CNS neurons: Prevention of perikaryal damage and death by ketamine. Brain Research, 543(2), 181-193.

86. Lucas, S. M., Rothwell, N. J., \& Gibson, R. M. (2006). The role of inflammation in CNS injury and disease. British Journal of Pharmacology, 147 Suppl 1, S232-40. doi:10.1038/sj.bjp.0706400

87. MacCumber, M. W., Ross, C. A., \& Snyder, S. H. (1990). Endothelin in brain: Receptors, mitogenesis, and biosynthesis in glial cells. Proceedings of the National Academy of Sciences of the United States of America, 87(6), 2359-2363.

88. Marar, M., Mcllvain, N. M., Fields, S. K., \& Comstock, R. D. (2012). Epidemiology of concussions among united states high school athletes in 20 sports. The American Journal of Sports Medicine, 40(4), 747-755. doi:10.1177/0363546511435626; 10.1177/0363546511435626

89. Margulies, S. S., \& Meaney, D. F. (1998). Brain tissues. In J. Black, \& G. Hastings (Eds.), Handbook of biomaterial properties (pp. 70-80). London: Chapman \& Hall.

90. Margulies, S. S., Thibault, L. E., \& Gennarelli, T. A. (1990). Physical model simulations of brain injury in the primate. Journal of Biomechanics, 23(8), 823-836.

91. McCracken, P. J., Manduca, A., Felmlee, J., \& Ehman, R. L. (2005). Mechanical transient-based magnetic resonance elastography. Magnetic Resonance in Medicine : Official Journal of the Society of Magnetic Resonance in Medicine / Society of Magnetic Resonance in Medicine, 53(3), 628-639. doi:10.1002/mrm.20388

92. Meaney, D. F., \& Thibault, L. E. (1990). Physical model studies of cortical brain deformation in response to high strain rate inertial loading. International IRCOBI Conference on the Biomechanics of Impacts, , 215-224.

93. Moon, Y., Kim, J. Y., Choi, S. Y., Kim, K., Kim, H., \& Sun, W. (2011). Induction of ezrin-radixin-moesin molecules after cryogenic traumatic brain injury of the mouse cortex. Neuroreport, 22(6), 304-308. doi:10.1097/WNR.0b013e3283460265; 10.1097/WNR.0b013e3283460265

94. Moore, S. W., Roca-Cusachs, P., \& Sheetz, M. P. (2010). Stretchy proteins on stretchy substrates: The important elements of integrin-mediated rigidity sensing. Developmental Cell, 19(2), 194-206. doi:10.1016/j.devcel.2010.07.018; 10.1016/j.devcel.2010.07.018

95. Moore, S. W., \& Sheetz, M. P. (2011). Biophysics of substrate interaction: Influence on neural motility, differentiation, and repair. Developmental Neurobiology, 71(11), 1090-1101. doi:10.1002/dneu.20947; 10.1002/dneu.20947

96. Morrison, B.,3rd, Cater, H. L., Benham, C. D., \& Sundstrom, L. E. (2006). An in vitro model of traumatic brain injury utilising two-dimensional stretch of organotypic hippocampal slice cultures. Journal of Neuroscience Methods, 150(2), 192-201. doi:10.1016/j.jneumeth.2005.06.014 
97. Morrison, B.,3rd, Cater, H. L., Wang, C. C., Thomas, F. C., Hung, C. T., Ateshian, G. A., \& Sundstrom, L. E. (2003). A tissue level tolerance criterion for living brain developed with an in vitro model of traumatic mechanical loading. Stapp Car Crash Journal, 47, 93-105.

98. Morrison, B.,3rd, Elkin, B. S., Dolle, J. P., \& Yarmush, M. L. (2011). In vitro models of traumatic brain injury. Annual Review of Biomedical Engineering, 13, 91-126. doi:10.1146/annurev-bioeng-071910124706; 10.1146/annurev-bioeng-071910-124706

99. Morrison, B.,3rd, Meaney, D. F., \& Mclntosh, T. K. (1998). Mechanical characterization of an in vitro device designed to quantitatively injure living brain tissue. Annals of Biomedical Engineering, 26(3), 381-390.

100. Morrison, B.,3rd, Saatman, K. E., Meaney, D. F., \& McIntosh, T. K. (1998). In vitro central nervous system models of mechanically induced trauma: A review. Journal of Neurotrauma, 15(11), 911-928.

101. Murphy, M. C., Huston, J.,3rd, Jack, C. R.,Jr, Glaser, K. J., Manduca, A., Felmlee, J. P., \& Ehman, R. L. (2011). Decreased brain stiffness in alzheimer's disease determined by magnetic resonance elastography. Journal of Magnetic Resonance Imaging : JMRI, 34(3), 494-498. doi:10.1002/jmri.22707; 10.1002/jmri.22707

102. Nakayama, Y., Aoki, Y., \& Niitsu, H. (2001). Studies on the mechanisms responsible for the formation of focal swellings on neuronal processes using a novel in vitro model of axonal injury. Journal of Neurotrauma, 18(5), 545-554. doi:10.1089/089771501300227341

103. Nawashiro, H., Messing, A., Azzam, N., \& Brenner, M. (1998). Mice lacking GFAP are hypersensitive to traumatic cerebrospinal injury. Neuroreport, 9(8), 1691-1696.

104. Newman, J. A. (1980). Head injury criteria in automotive crash testing. Twenty-Fourth Stapp Car Crash Conference, Ottawa, Ontario, Cananda. 704-747.

105. Newman, J. A. (1998). Kinematics of head injury. In N. Yoganandan (Ed.), Frontiers in head and neck trauma (pp. 200) IOS Press.

106. Newman, J. A., Barr, C., Beusenberg, M., Fournier, E., Shewchenko, N., Welbourne, E., \& Withnall, C. (2000). A new biomechanical assessment of mild traumatic brain injury part 2- results and conclusions. International Research Council on the Biomechanics of Impact, Montpellier, France. 223-233.

107. Newman, J. A., Beusenberg, M., Fournier, E., Shewchenko, N., Withnall, C., King, A., .. McGinnis, G. (1999). "A new biomechanical assessment of mild traumatic brain injury, part 1-methodology. International Research Council on Biomechanics of Impact, Barcelona, Spain. 17-36.

108. Ommaya, A. K., Corrao, P., \& Letcher, F. S. (1973). Head injury in the chimpanzee. 1. biodynamics of traumatic unconsciousness. Journal of Neurosurgery, 39(2), 152-166. doi:10.3171/jns.1973.39.2.0152

109. Ommaya, A. K., Goldsmith, W., \& Thibault, L. (2002). Biomechanics and neuropathology of adult and paediatric head injury. British Journal of Neurosurgery, 16(3), 220-242.

110. Ostrow, L. W., Langan, T. J., \& Sachs, F. (2000). Stretch-induced endothelin-1 production by astrocytes. Journal of Cardiovascular Pharmacology, 36(5 Suppl 1), S274-7. 
111. Pekny, M., \& Wilhelmsson, U. (2006). GFAP and astrocyte intermediate filaments. In A. Laitha (Ed.), Handbook of neurochemistry and molecular neurobiology, neuroactive proteins and peptides (pp. 289-314)

112. Pellman, E. J., Viano, D. C., Tucker, A. M., Casson, I. R., \& Waeckerle, J. F. (2003). Concussion in professional football: Reconstruction of game impacts and injuries. Neurosurgery, 53(4), 799-812; discussion 812-4.

113. Pham, N., Fazio, V., Cucullo, L., Teng, Q., Biberthaler, P., Bazarian, J. J., \& Janigro, D. (2010). Extracranial sources of S100B do not affect serum levels. PloS One, 5(9), 10.1371/journal.pone.0012691. doi:10.1371/journal.pone.0012691; 10.1371/journal.pone.0012691

114. Prange, M., \& Margulies, S. S. (2001). Tissue strain thresholds for axonal injury in the infant brain. American Society of Mechanical Engineers Bioengineering Conference, 50, 833.

115. Prange, M. T., \& Margulies, S. S. (2002). Regional, directional, and age-dependent properties of the brain undergoing large deformation. Journal of Biomechanical Engineering, 124(2), 244-252.

116. Raabe, A., Grolms, C., \& Seifert, V. (1999). Serum markers of brain damage and outcome prediction in patients after severe head injury. British Journal of Neurosurgery, 13(1), 56-59.

117. Rader, D. J. (2006). Molecular regulation of HDL metabolism and function: Implications for novel therapies. The Journal of Clinical Investigation, 116(12), 3090-3100. doi:10.1172/JCI30163

118. Raghupathi, R. (2004). Cell death mechanisms following traumatic brain injury. Brain Pathology (Zurich, Switzerland), 14(2), 215-222.

119. Ridet, J. L., Malhotra, S. K., Privat, A., \& Gage, F. H. (1997). Reactive astrocytes: Cellular and molecular cues to biological function. Trends in Neurosciences, 20(12), 570-577.

120. Rogers, S. D., Demaster, E., Catton, M., Ghilardi, J. R., Levin, L. A., Maggio, J. E., \& Mantyh, P. W. (1997). Expression of endothelin-B receptors by glia in vivo is increased after CNS injury in rats, rabbits, and humans. Experimental Neurology, 145(1), 180-195. doi:10.1006/exnr.1997.6468

121. Rothoerl, R. D., Woertgen, C., Holzschuh, M., Metz, C., \& Brawanski, A. (1998). S-100 serum levels after minor and major head injury. The Journal of Trauma, 45(4), 765-767.

122. Ruoslathi, E. (1996). Brain extracellular matrix.6(5), 489-492.

123. Sack, I., Streitberger, K. J., Krefting, D., Paul, F., \& Braun, J. (2011). The influence of physiological aging and atrophy on brain viscoelastic properties in humans. PloS One, 6(9), e23451. doi:10.1371/journal.pone.0023451; 10.1371/journal.pone.0023451

124. Saunders, R. L., \& Harbaugh, R. E. (1984). The second impact in catastrophic contact-sports head trauma. JAMA : The Journal of the American Medical Association, 252(4), 538-539.

125. Schulz, M. R., Marshall, S. W., Mueller, F. O., Yang, J., Weaver, N. L., Kalsbeek, W. D., \& Bowling, J. M. (2004). Incidence and risk factors for concussion in high school athletes, north carolina, 19961999. American Journal of Epidemiology, 160(10), 937-944. doi:10.1093/aje/kwh304

126. Shaw, N. A. (2002). The neurophysiology of concussion. Progress in Neurobiology, 67(4), 281-344. 
127. Shreiber, D. I., Smith, D. H., \& Meaney, D. F. (1999). Immediate in vivo response of the cortex and the blood-brain barrier following dynamic cortical deformation in the rat. Neuroscience Letters, 259(1), 5-8.

128. Siren, A. L., Knerlich, F., Schilling, L., Kamrowski-Kruck, H., Hahn, A., \& Ehrenreich, H. (2000). Differential glial and vascular expression of endothelins and their receptors in rat brain after neurotrauma. Neurochemical Research, 25(7), 957-969.

129. Sofroniew, M. V., \& Vinters, H. V. (2010). Astrocytes: Biology and pathology. Acta Neuropathologica, 119(1), 7-35. doi:10.1007/s00401-009-0619-8; 10.1007/s00401-009-0619-8

130. Tavazzi, B., Vagnozzi, R., Signoretti, S., Amorini, A. M., Belli, A., Cimatti, M., . . Lazzarino, G. (2007). Temporal window of metabolic brain vulnerability to concussions: Oxidative and nitrosative stresses--part II. Neurosurgery, 61(2), 390-5; discussion 395-6.

131. Thomas, L. M., Roberts, V. L., \& Gurdjian, E. S. (1966). Experimental intracranial pressure gradients in the human skull. Journal of Neurology, Neurosurgery, and Psychiatry, 29(5), 404-411.

132. Thurman, D. J., Branche, C. M., \& Sniezek, J. E. (1998). The epidemiology of sports-related traumatic brain injuries in the united states: Recent developments. The Journal of Head Trauma Rehabilitation, 13(2), 1-8.

133. Tsang, M. C., Lo, A. C., Cheung, P. T., Chung, S. S., \& Chung, S. K. (2001). Perinatal hypoxia/ischemia-induced endothelin-1 mRNA in astrocyte-like and endothelial cells. Neuroreport, 12(10), 2265-2270.

134. Tyler, W. J. (2012). The mechanobiology of brain function. Nature Reviews. Neuroscience, 13(12), 867-878. doi:10.1038/nrn3383; 10.1038/nrn3383

135. Vagnozzi, R., Tavazzi, B., Signoretti, S., Amorini, A. M., Belli, A., Cimatti, M., . . Lazzarino, G. (2007). Temporal window of metabolic brain vulnerability to concussions: Mitochondrial-related impairment--part I. Neurosurgery, 61(2), 379-88; discussion 388-9.

doi:10.1227/01.NEU.0000280002.41696.D8

136. Van Lenten, B. J., Reddy, S. T., Navab, M., \& Fogelman, A. M. (2006). Understanding changes in high density lipoproteins during the acute phase response. Arteriosclerosis, Thrombosis, and Vascular Biology, 26(8), 1687-1688. doi:10.1161/01.ATV.0000232522.47018.a6

137. Viano, D. C., Hamberger, A., Bolouri, H., \& Saljo, A. (2009). Concussion in professional football: Animal model of brain injury--part 15. Neurosurgery, 64(6), 1162-73; discussion 1173. doi:10.1227/01.NEU.0000345863.99099.C7; 10.1227/01.NEU.0000345863.99099.C7

138. Woertgen, C., Rothoerl, R. D., Metz, C., \& Brawanski, A. (1999). Comparison of clinical, radiologic, and serum marker as prognostic factors after severe head injury. The Journal of Trauma, 47(6), 1126-1130.

139. Wolf, J. A., Stys, P. K., Lusardi, T., Meaney, D., \& Smith, D. H. (2001). Traumatic axonal injury induces calcium influx modulated by tetrodotoxin-sensitive sodium channels. The Journal of Neuroscience : The Official Journal of the Society for Neuroscience, 21(6), 1923-1930.

140. Wuerfel, J., Paul, F., Beierbach, B., Hamhaber, U., Klatt, D., Papazoglou, S., . . Sack, I. (2010). MR-elastography reveals degradation of tissue integrity in multiple sclerosis. Neurolmage, 49(3), 2520-2525. doi:10.1016/j.neuroimage.2009.06.018; 10.1016/j.neuroimage.2009.06.018 
141. Zhang, J., Green, M. A., Sinkus, R., \& Bilston, L. E. (2011). Viscoelastic properties of human cerebellum using magnetic resonance elastography. Journal of Biomechanics, 44(10), 1909-1913. doi:10.1016/j.jbiomech.2011.04.034; 10.1016/j.jbiomech.2011.04.034 


\section{APPENDIX A - Matlab Code}

clc

clear all

close all

imtool close all

$\% \%$ Reads Images

\%image is from Dropbox $>$ MATLAB $>$ TSVIEW $>$ Strain > 7_27_2012 folder

$\% 700$ microliter of $6 \mathrm{mg} / \mathrm{mL}$ (microspheres/alcohol) and dried overnight

cntrl0=imadjust(rgb2gray(imread('816_0ticks_control.jpg')));

cntrl1=imadjust(rgb2gray(imread('816_1ticks_control.jpg')));

cntrl2=imadjust(rgb2gray(imread('816_2ticks_control.jpg')));

cntrl3=imadjust(rgb2gray(imread('816_3ticks_control.jpg')));

cntrl4=imadjust(rgb2gray(imread('816_4ticks_control.jpg')));

cntrl5=imadjust(rgb2gray(imread('816_5ticks_control.jpg')));

dfrm0=imadjust(rgb2gray(imread('816_0ticks.jpg')));

dfrm1=imadjust(rgb2gray(imread('816_1ticks.jpg')));

dfrm2=imadjust(rgb2gray(imread('816_2ticks.jpg')));

dfrm3=imadjust(rgb2gray(imread('816_3ticks.jpg')));

dfrm4=imadjust(rgb2gray(imread('816_4ticks.jpg')));

dfrm5=imadjust(rgb2gray(imread('816_5ticks.jpg')));

$\% \%$ IMTOOL

imtool close all

\%figure(1), subplot(2,3,1), imshow(cntrl0), subplot(2,3,2), imshow(cntrl1), subplot(2,3,3), imshow(cntrl2), ..

$\%$ subplot( $2,3,4)$, imshow(cntrl3), subplot( $2,3,5)$, imshow(cntrl4), subplot(2,3,6), imshow(cntrl5)

\%figure(2), subplot(1,2,1), imshow(a1),subplot(1,2,2), imshow(b1)

$\%$ hold on

$\% \operatorname{plot}\left(1: \operatorname{size}(\mathrm{a} 1,2), \operatorname{size}(\mathrm{a} 1,1)^{*} 0.5\right.$, 'r-' $\left.^{\prime}\right)$

$\%$ hold off

\%imtool(cntrl0)

$\%$ imtool(dfrm0)

$\% \%$ Manual bead centroid recording

$\% \mathrm{~A} / \mathrm{B} / \mathrm{C}$ are undeformed points; $\mathrm{A} 1 / \mathrm{B} 1 / \mathrm{C} 1$ are deformed points

$\% \mathrm{~A} / \mathrm{A} 1$ is farthest to the left

$\% \mathrm{~B} / \mathrm{B} 1$ is middle and farthest from $\mathrm{A}$ and $\mathrm{C}$

$\% \mathrm{C} / \mathrm{C} 1$ is farthest right

$\%$ the pixel coordinate system has $\mathrm{x}$ axis pointing right and the $\mathrm{y}$ axis

$\%$ pointing down. 'imread' function starts reading pixel intensities from the

\%top down and left to right.

\%Beads are chosen that are not part of big cluster.

$\% \% 11.95 \mathrm{~mm}$

$\%$ cntrl0/dfrm0

$\%$ a1 $=[672,470]$;

$\%$ b1 $=[945,787] ;$

$\% c 1=[1141,547]$ 


$$
\begin{aligned}
& \% A 1=[100,261] ; \\
& \% \mathrm{~B} 1=[432,657] \text {; } \\
& \% \mathrm{C} 1=[678,367] \text {; } \\
& \% a 2=[718,467] \text {; } \\
& \% b 2=[1012,313] \text {; } \\
& \% c 2=[1141,547] \text {; } \\
& \% A 2=[157,258] \text {; } \\
& \% B 2=[525,78] \text {; } \\
& \% \mathrm{C} 2=[678,367] \text {; } \\
& \% \text { a3=[639,566]; } \\
& \% b 3=[782,889] \text {; } \\
& \% c 3=[1008,664] \text {; } \\
& \% A 3=[51,376] \text {; } \\
& \% B 3=[228,773] \text {; } \\
& \% \mathrm{C} 3=[504,504] \text {; } \\
& \% \text { cntrl1/dfrm1 } \\
& \% \text { a1=[854,569]; } \\
& \% b 1=[956,300] \text {; } \\
& \text { \%c1=[1155,594]; } \\
& \% \mathrm{~A} 1=[377,468] \text {; } \\
& \% B 1=[499,140] \text {; } \\
& \% \mathrm{C} 1=[736,494] \text {; } \\
& \% \text { a2=[661,812]; } \\
& \% b 2=[854,569] \text {; } \\
& \% c 2=[961,842] \text {; } \\
& \% A 2=[140,767] \text {; } \\
& \% \mathrm{~B} 2=[377,468] \text {; } \\
& \% C 2=[504,799] \text {; } \\
& \% \text { a3=[743,345]; } \\
& \% b 3=[854,569] \text {; } \\
& \% c 3=[1032,378] \text {; } \\
& \% A 3=[243,196] ; \\
& \% \mathrm{~B} 3=[377,468] \text {; } \\
& \% \text { C3=[590,236]; } \\
& \text { \%cntrl2/dfrm2 } \\
& \% \text { a1=[839,269]; } \\
& \% \text { b1 }=[977,638] \text {; } \\
& \% c 1=[1223,342] \text {; } \\
& \% A 1=[435,133] \text {; } \\
& \% \mathrm{~B} 1=[604,578] \text {; } \\
& \% \mathrm{C1}=[896,219] \text {; }
\end{aligned}
$$




$$
\begin{aligned}
& \% a 2=[843,398] \text {; } \\
& \% \text { b2=[861,607]; } \\
& \% c 2=[1076,505] \text {; } \\
& \% A 2=[441,289] \text {; } \\
& \% \mathrm{~B} 2=[466,543] \text {; } \\
& \% C 2=[724,414] \text {; } \\
& \% \text { a3=[526,248]; } \\
& \% b 3=[715,545] \text {; } \\
& \% c 3=[838,270] \text {; } \\
& \% A 3=[58,114] \text {; } \\
& \% B 3=[288,468] \text {; } \\
& \% \mathrm{C} 3=[436,134] \text {; } \\
& \% \text { cntrl3/dfrm3 } \\
& \% \text { a1=[736,281]; } \\
& \% b 1=[886,565] \text {; } \\
& \% c 1=[1086,301] \text {; } \\
& \% A 1=[398,98] \text {; } \\
& \% \mathrm{~B} 1=[577,441] \text {; } \\
& \% \mathrm{C} 1=[813,124] \text {; } \\
& \% a 2=[588,553] \text {; } \\
& \% b 2=[736,281] \text {; } \\
& \% c 2=[831,568] \text {; } \\
& \% A 2=[215,424] \text {; } \\
& \% B 2=[398,98] \text {; } \\
& \% C 2=[514,444] \text {; } \\
& \% \text { a3=[728,390]; } \\
& \% b 3=[847,589] \text {; } \\
& \% c 3=[976,395] \text {; } \\
& \% A 3=[387,229] \text {; } \\
& \% B 3=[533,471] \text {; } \\
& \% \text { C3=[689,236]; }
\end{aligned}
$$

\%the $y$ or $x$ axis of the pixel coordinate system is parallel with

$\%$ the radial axis of the beads. The radial axis is determined as the middle $\%$ plane in the image because it was centered from the edges of the indentor $\%$ at zoom 5 (a rough estimation as to where the curve of the ID was greatest $\%$ and positioning that point in the middle of the image). Therefore any \%beads in the middle of the image is already on its radial axis which is $\%$ also parallel with one of the pixel coordinate axes.

$\% 7 / 30 / 2012$

$\%$ Movable microscope stage fixated by tape to protective white poster board on dissection scope platform.

$\%$ As before, an edge of the stage was used to square the stage with the camera. Pixel distances from the stage's edge to... 
$\%$ the end of the image area was taken to determine the final position of the $\%$ movable stage.

$\%$ At zoom 4, the ID of the indentor edge where it had its greatest concavity $\%$ was approximated at the center horizontal line of the image.

$\% \%$ Line Lengths between dots

$\%$ Undeformed

$\mathrm{ab} 1=\left((\mathrm{abs}(\mathrm{a} 1(1)-\mathrm{b} 1(1))) \cdot{ }^{\wedge} 2+(\mathrm{abs}(\mathrm{a} 1(2)-\mathrm{b} 1(2))) \cdot{ }^{\wedge} 2\right) \cdot{ }^{\wedge} 0.5$;

$\mathrm{bc} 1=\left((\operatorname{abs}(\mathrm{b} 1(1)-\mathrm{c} 1(1))) \cdot{ }^{\wedge} 2+(\operatorname{abs}(\mathrm{b} 1(2)-\mathrm{c} 1(2))) \cdot{ }^{\wedge} 2\right) \cdot{ }^{\wedge} 0.5$;

ca1 $=\left((\operatorname{abs}(\mathrm{c} 1(1)-\mathrm{a} 1(1))) \cdot{ }^{\wedge} 2+(\operatorname{abs}(\mathrm{c} 1(2)-\mathrm{a} 1(2))) \cdot{ }^{\wedge} 2\right) \cdot{ }^{\wedge} 0.5$

$\mathrm{ab} 2=\left((\mathrm{abs}(\mathrm{a} 2(1)-\mathrm{b} 2(1))) \cdot{ }^{\wedge} 2+(\mathrm{abs}(\mathrm{a} 2(2)-\mathrm{b} 2(2))) \cdot{ }^{\wedge} 2\right) .{ }^{\wedge} 0.5$;

$\mathrm{bc}=\left((\mathrm{abs}(\mathrm{b} 2(1)-\mathrm{c} 2(1))) \cdot{ }^{\wedge} 2+(\mathrm{abs}(\mathrm{b} 2(2)-\mathrm{c} 2(2))) \cdot{ }^{\wedge} 2\right) \cdot{ }^{\wedge} 0.5$;

$\mathrm{ca} 2=\left((\operatorname{abs}(\mathrm{c} 2(1)-\mathrm{a} 2(1))) \cdot{ }^{\wedge} 2+(\operatorname{abs}(\mathrm{c} 2(2)-\mathrm{a} 2(2))) \cdot{ }^{\wedge} 2\right) \cdot{ }^{\wedge} 0.5$;

$a b 3=\left((\operatorname{abs}(a 3(1)-b 3(1))) \cdot{ }^{\wedge} 2+(\operatorname{abs}(a 3(2)-b 3(2))) \cdot \wedge^{\wedge} 2\right) \cdot{ }^{\wedge} 0.5$;

$\mathrm{bc} 3=\left((\operatorname{abs}(b 3(1)-c 3(1))) \cdot{ }^{\wedge} 2+(\operatorname{abs}(b 3(2)-c 3(2))) \cdot{ }^{\wedge} 2\right) \cdot{ }^{\wedge} 0.5 ;$

$\mathrm{ca} 3=\left((\operatorname{abs}(\mathrm{c} 3(1)-\mathrm{a} 3(1))) \cdot{ }^{\wedge} 2+(\operatorname{abs}(\mathrm{c} 3(2)-\mathrm{a} 3(2))) \cdot{ }^{\wedge} 2\right) \cdot{ }^{\wedge} 0.5$;

$\%$ Deformed

$\mathrm{AB} 1=\left((\operatorname{abs}(\mathrm{A} 1(1)-\mathrm{B} 1(1))) \cdot{ }^{\wedge} 2+(\operatorname{abs}(\mathrm{A} 1(2)-\mathrm{B} 1(2))) \cdot{ }^{\wedge} 2\right) \cdot{ }^{\wedge} 0.5$;

$B C 1=\left((\operatorname{abs}(B 1(1)-C 1(1))) \cdot{ }^{\wedge} 2+(\operatorname{abs}(B 1(2)-C 1(2))) \cdot{ }^{\wedge} 2\right) \cdot{ }^{\wedge} 0.5$;

$\mathrm{CA} 1=\left((\operatorname{abs}(\mathrm{C} 1(1)-\mathrm{A} 1(1))) \cdot{ }^{\wedge} 2+(\operatorname{abs}(\mathrm{C} 1(2)-\mathrm{A} 1(2))) \cdot{ }^{\wedge} 2\right) \cdot{ }^{\wedge} 0.5$;

$\mathrm{AB} 2=\left((\operatorname{abs}(\mathrm{A} 2(1)-\mathrm{B} 2(1))) \cdot{ }^{\wedge} 2+(\operatorname{abs}(\mathrm{A} 2(2)-\mathrm{B} 2(2))) \cdot{ }^{\wedge} 2\right) \cdot{ }^{\wedge} 0.5$;

$\mathrm{BC} 2=\left((\operatorname{abs}(\mathrm{B} 2(1)-\mathrm{C} 2(1))) \cdot{ }^{\wedge} 2+(\operatorname{abs}(\mathrm{B} 2(2)-\mathrm{C} 2(2))) \cdot{ }^{\wedge} 2\right) \cdot{ }^{\wedge} 0.5$;

$\mathrm{CA} 2=\left((\operatorname{abs}(\mathrm{C} 2(1)-\mathrm{A} 2(1))) \cdot{ }^{\wedge} 2+(\operatorname{abs}(\mathrm{C} 2(2)-\mathrm{A} 2(2))) \cdot{ }^{\wedge} 2\right) \cdot{ }^{\wedge} 0.5$;

$\mathrm{AB} 3=\left((\operatorname{abs}(\mathrm{A} 3(1)-\mathrm{B} 3(1))) \cdot{ }^{\wedge} 2+(\operatorname{abs}(\mathrm{A} 3(2)-\mathrm{B} 3(2))) \cdot{ }^{\wedge} 2\right) \cdot{ }^{\wedge} 0.5$;

$\mathrm{BC} 3=\left((\operatorname{abs}(\mathrm{B} 3(1)-\mathrm{C} 3(1))) \cdot{ }^{\wedge} 2+(\operatorname{abs}(\mathrm{B} 3(2)-\mathrm{C} 3(2))) \cdot{ }^{\wedge} 2\right) \cdot{ }^{\wedge} 0.5$;

$\mathrm{CA} 3=\left((\operatorname{abs}(\mathrm{C} 3(1)-\mathrm{A} 3(1))) \cdot{ }^{\wedge} 2+(\operatorname{abs}(\mathrm{C} 3(2)-\mathrm{A} 3(2))) \cdot{ }^{\wedge} 2\right) \cdot{ }^{\wedge} 0.5$;

$\%$ Between the deformed and undeformed dots

$\% A \_A 1=\left((\operatorname{abs}(A 1(1)-A(1))) \cdot{ }^{\wedge} 2+(\operatorname{abs}(A 1(2)-A(2))) \cdot{ }^{\wedge} 2\right) \cdot{ }^{\wedge} 0.5$;

$\% B \_B 1=\left((\operatorname{abs}(B 1(1)-B(1))) \cdot{ }^{\wedge} 2+(\operatorname{abs}(B 1(2)-B(2))) \cdot{ }^{\wedge} 2\right) \cdot{ }^{\wedge} 0.5$;

$\% \mathrm{C} \_\mathrm{C} 1=\left((\operatorname{abs}(\mathrm{C} 1(1)-\mathrm{C}(1))) \cdot{ }^{\wedge} 2+(\operatorname{abs}(\mathrm{C} 1(2)-\mathrm{C}(2))) \cdot{ }^{\wedge} 2\right) \cdot{ }^{\wedge} 0.5$;

\%d_u_dist=cat(1,A_A1,B_B1,C_C1)

\%above no longer applies because I picked up the PDMS holder to place

\%indentor underneath

$\%$ Would it matter at all as long as I have the deformed

$\%$ coordinates and made sure the centerlines were leveled properly?

$\% \%$ The Lagrangian strain tensor

$\%$ delta= double_Lagrangian * components

delta1 $=\left[\mathrm{AB} 1 .^{\wedge} 2-\mathrm{ab} 1 .{ }^{\wedge} 2 ; \mathrm{BC} 1 .^{\wedge} 2-\mathrm{bc} 1 .^{\wedge} 2 ; \mathrm{CA} 1 .{ }^{\wedge} 2-\mathrm{ca} 1 .^{\wedge} 2\right] ;$

delta2 $=\left[\mathrm{AB} 2 .^{\wedge} 2-\mathrm{ab} 2 .{ }^{\wedge} 2 ; \mathrm{BC} 2 .^{\wedge} 2-\mathrm{bc} 2 .^{\wedge} 2 ; \mathrm{CA} 2 .^{\wedge} 2-\mathrm{ca} 2 .^{\wedge} 2\right]$;

delta3=[AB3.^2-ab3.^2; BC3.^2-bc3.^2; CA3.^2-ca3.^2];

$\%$ delta is the difference in length between each deformed and undeformed line

\%segments: $A B, B C$, and $C A$

$\%$ The two dimensional components of the undeformed line segment in the referenced plane $\%$ LineAB 


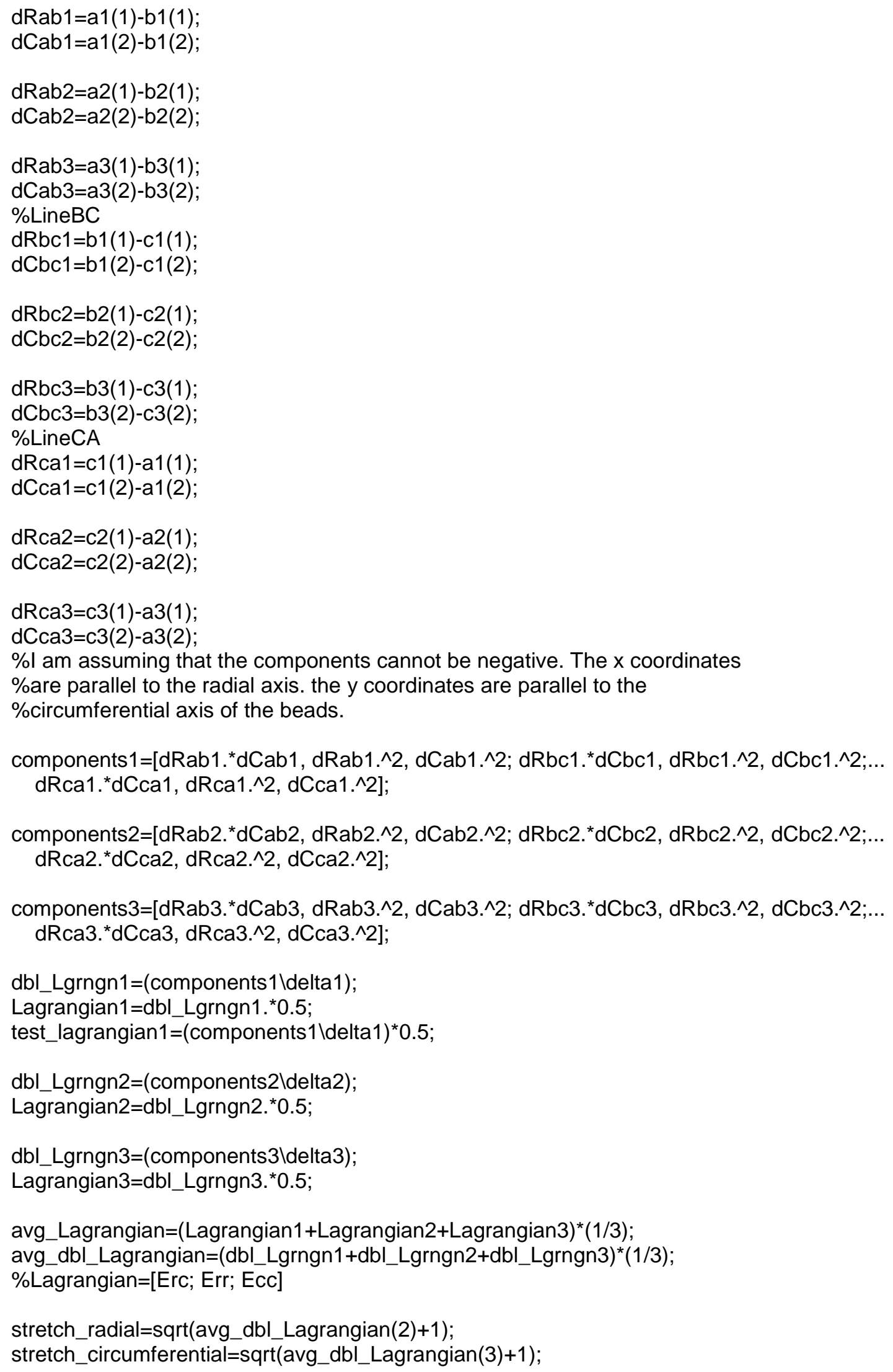


\%stretch is the stretch ratio

delta_angle=asin(avg_dbl_Lagrangian(1)./(stretch_radial+stretch_circumferential) $)^{*}(180 / p i)$;

Inr_strain_radial=stretch_radial-1;

Inr_strain_circumferential=stretch_circumferential-1;

$\% \%$ Excel analysis of triangle arrangements (orientation, perimeter, and equilibrium)

$\%$ and Lagrangian strain for each triangle at each position.

$\% \% \% \% \%$ orientation of the line relative to the horizontal pixel $(\mathrm{x})$ axis pointing right of $\% \% \% \% \%$ image (0 ---> 1280). Positive angles are counter clock wise.

theta_ab1=atan $((a 1(2)-b 1(2)) /(a 1(1)-b 1(1)))^{*}(180 / p i)$;

theta_bc1 =atan $((\mathrm{b} 1(2)-\mathrm{c} 1(2)) /(\mathrm{b} 1(1)-\mathrm{c} 1(1)))^{*}(180 / \mathrm{pi})$;

theta_ac1=atan $((\mathrm{a} 1(2)-\mathrm{c} 1(2)) /(\mathrm{a} 1(1)-\mathrm{c} 1(1)))^{*}(180 / \mathrm{pi})$;

theta_AB1 $=\operatorname{atan}(\operatorname{abs}(\mathrm{A} 1(2)-\mathrm{B} 1(2)) / \mathrm{abs}(\mathrm{A} 1(1)-\mathrm{B} 1(1)))^{*}(180 / \mathrm{pi})$;

theta_ab2=atan $((a 2(2)-b 2(2)) /(a 2(1)-b 2(1)))^{*}(180 / p i)$;

theta_bc2=atan $((b 2(2)-c 2(2)) /(b 2(1)-c 2(1)))^{*}(180 / p i)$;

theta_ac2=atan $((\mathrm{a} 2(2)-\mathrm{c} 2(2)) /(\mathrm{a} 2(1)-\mathrm{c} 2(1)))^{*}(180 / \mathrm{pi})$;

theta_ab3=atan $((a 3(2)-b 3(2)) /(a 3(1)-b 3(1)))^{*}(180 / p i)$;

theta_bc3=atan $((b 3(2)-c 3(2)) /(b 3(1)-c 3(1)))^{*}(180 / p i)$;

theta_ac3=atan $((a 3(2)-c 3(2)) /(a 3(1)-c 3(1)))^{*}(180 / p i)$;

theta_all=[theta_ab1 theta_ab2 theta_ab3;theta_bc1 theta_bc2 theta_bc3;theta_ac1 theta_ac2 theta_ac3]

perimeter $=[a b 1+b c 1+c a 1, a b 2+b c 2+c a 2, a b 3+b c 3+c a 3]$

sides=[ab1 ab2 ab3;bc1 bc2 bc3;ca1 ca2 ca3]

$\% \% \% \% \%$ determining range of triangle side lengths for each arrangement

delta_sides $=\max ($ sides,[],1) $-\min ($ sides,[],1)

$\% \% \% \% \%$ estimation of positional rotation while manually deforming.

$\%$ rotation=abs (theta_AB1)-abs(theta_ab1)

Lagrangian=cat(2,Lagrangian1, Lagrangian2,Lagrangian3) 
XI. APPENDIX B - Drawings

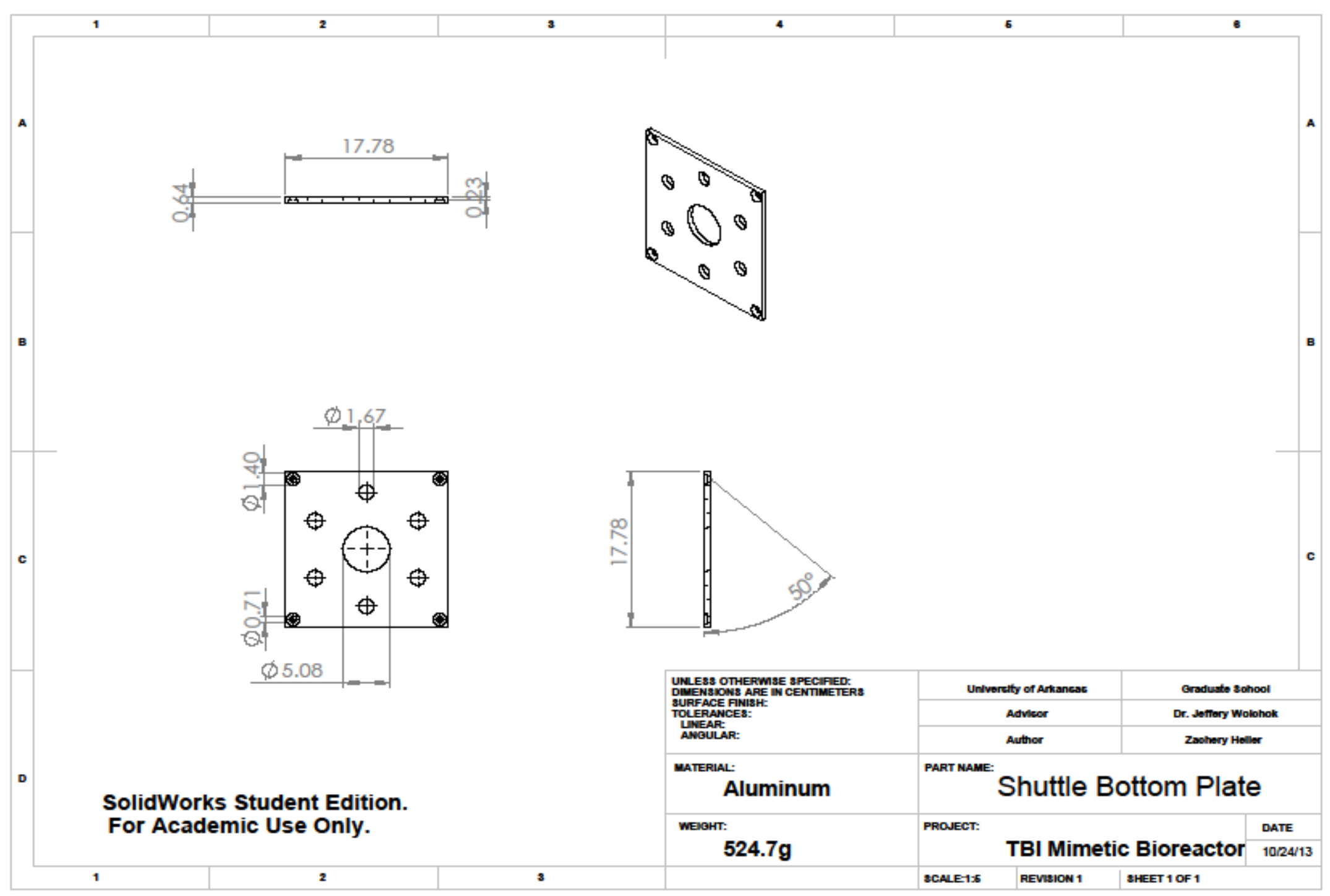




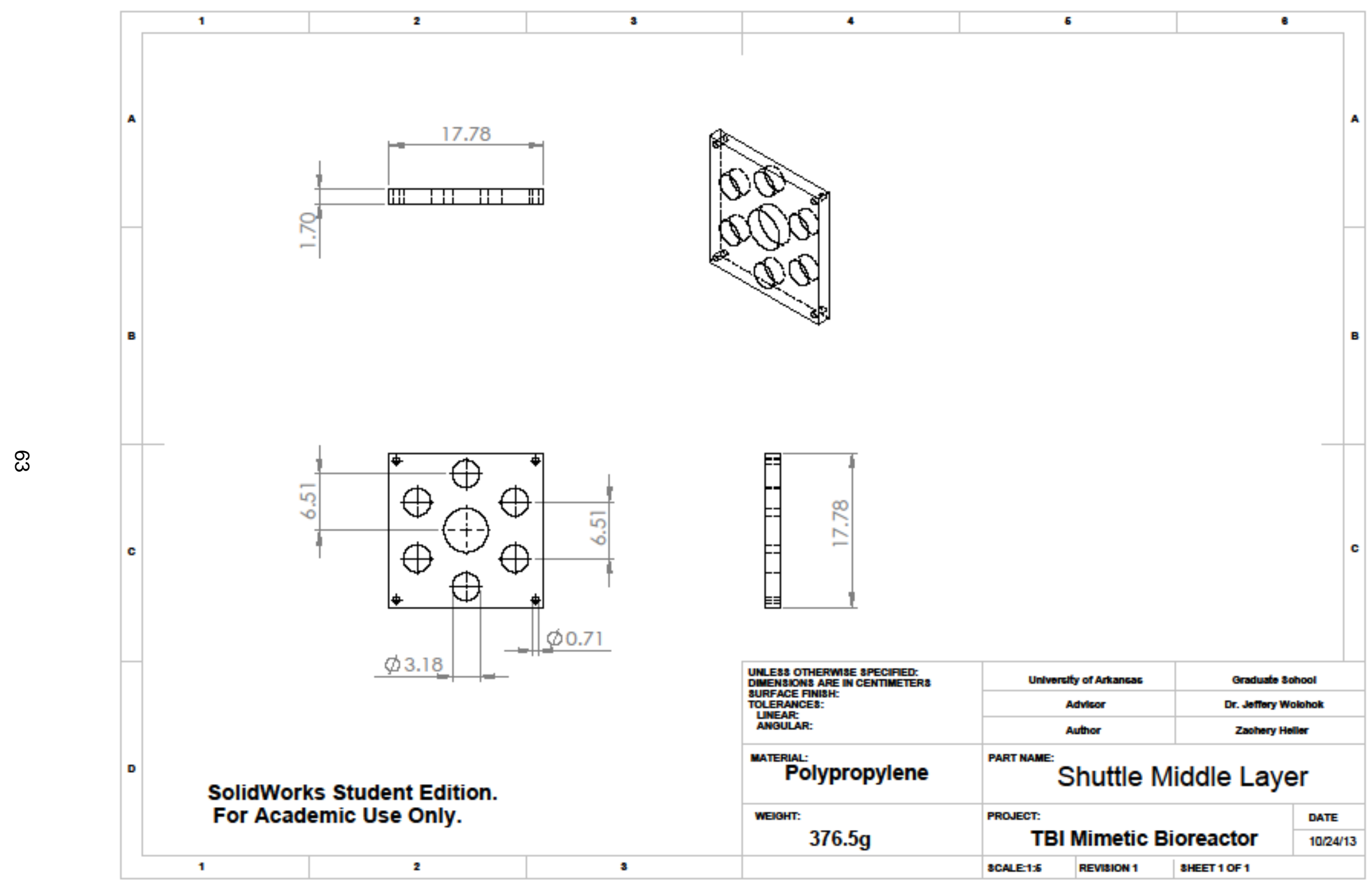




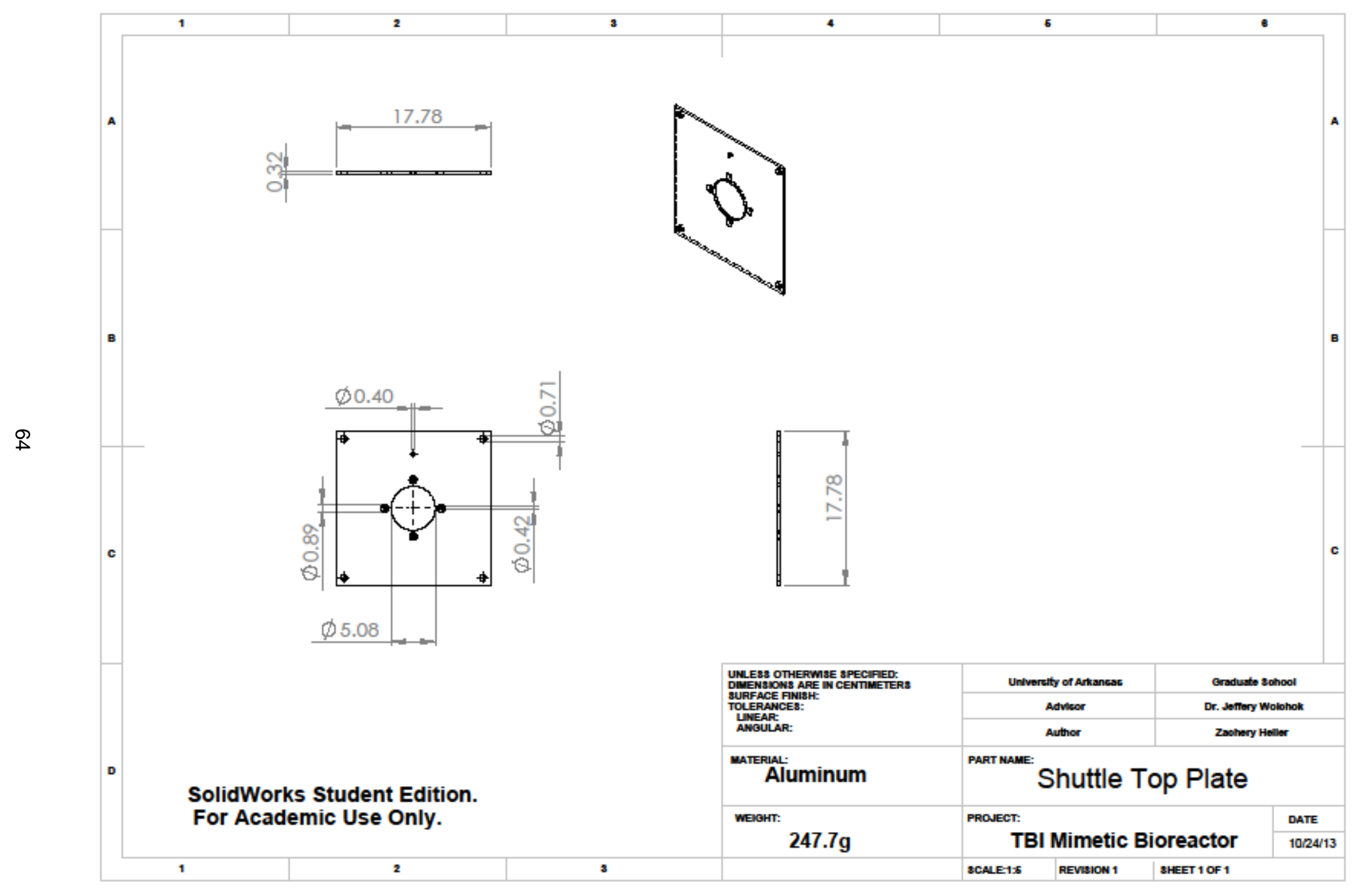




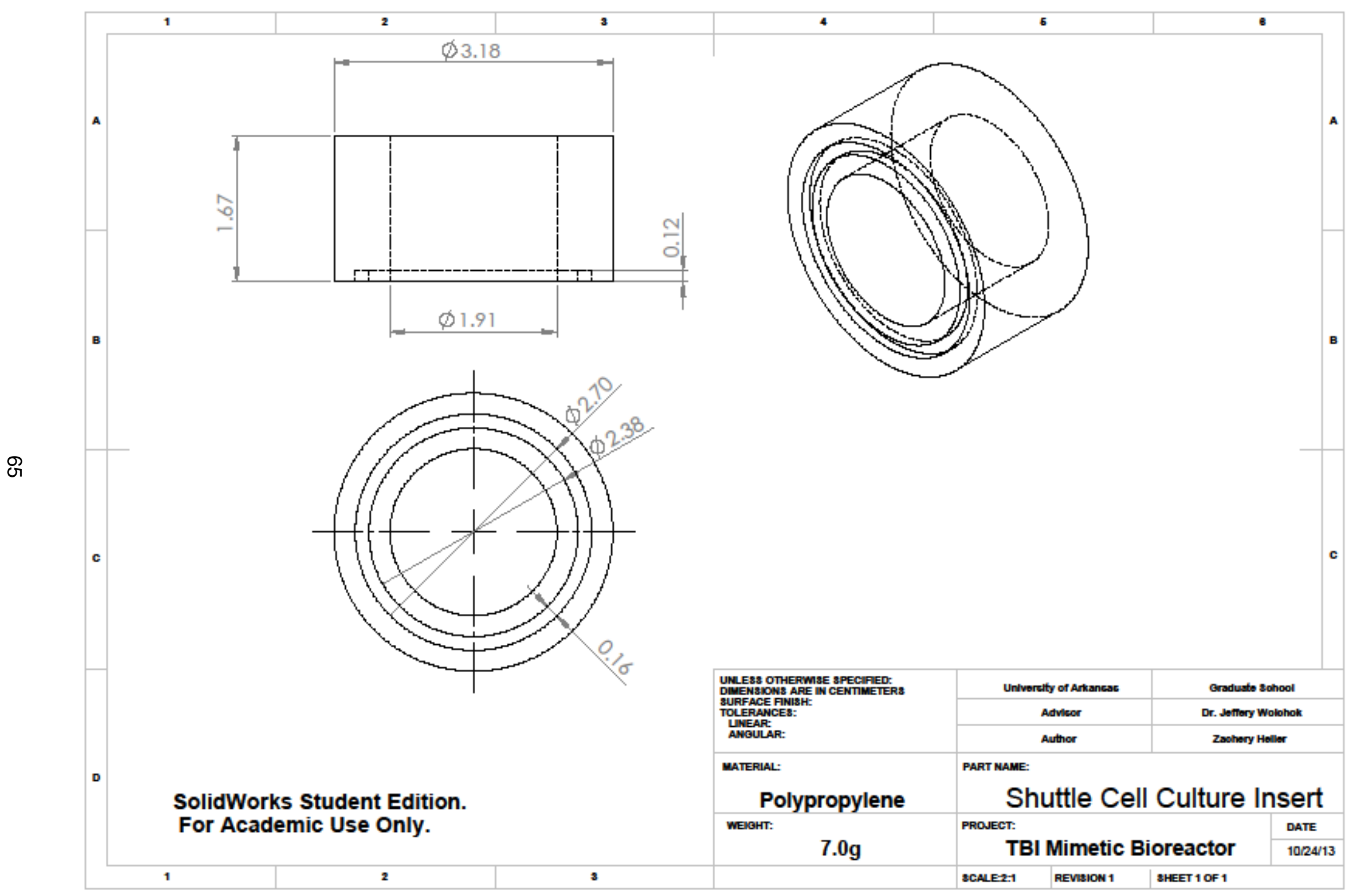




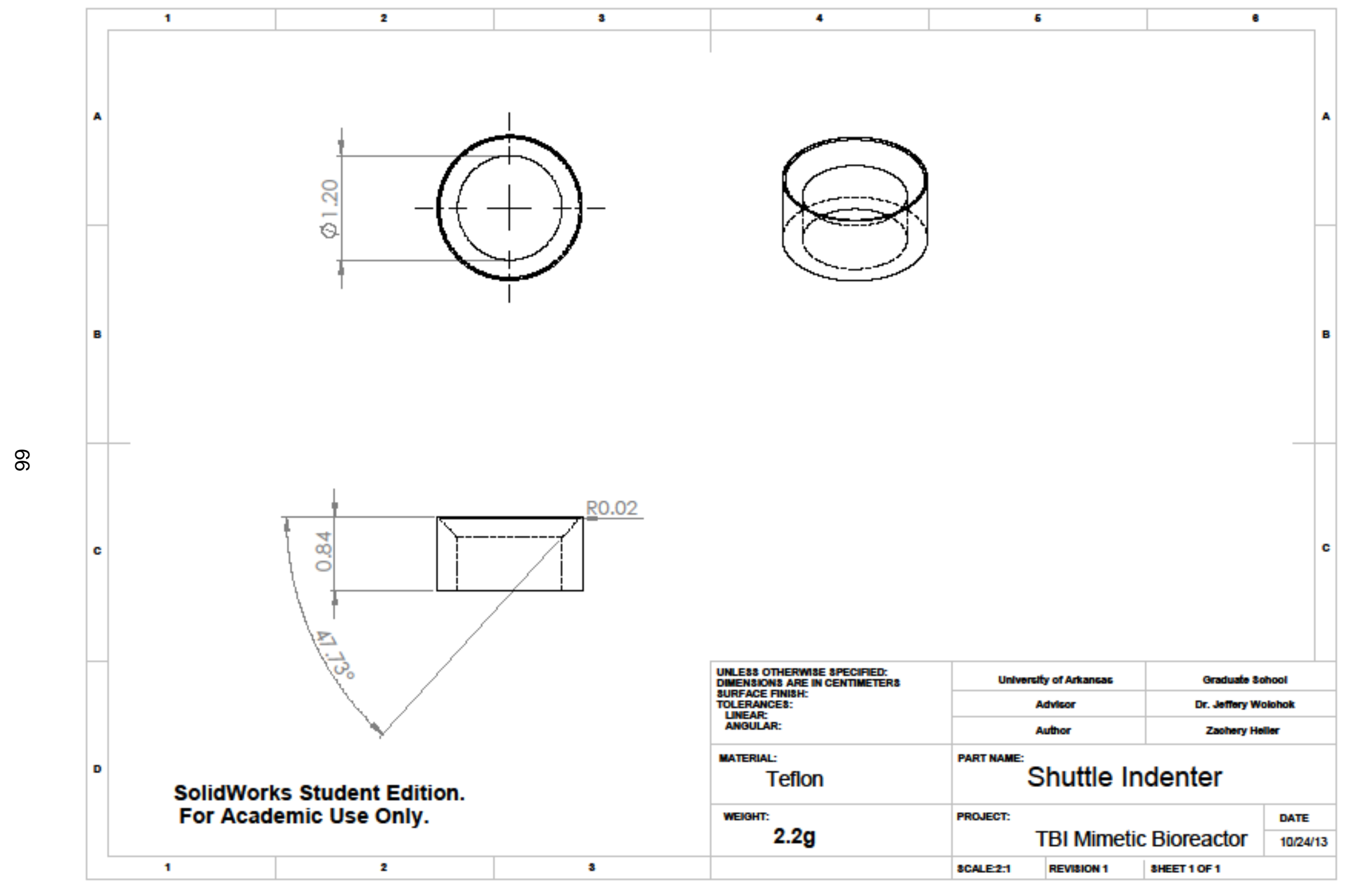




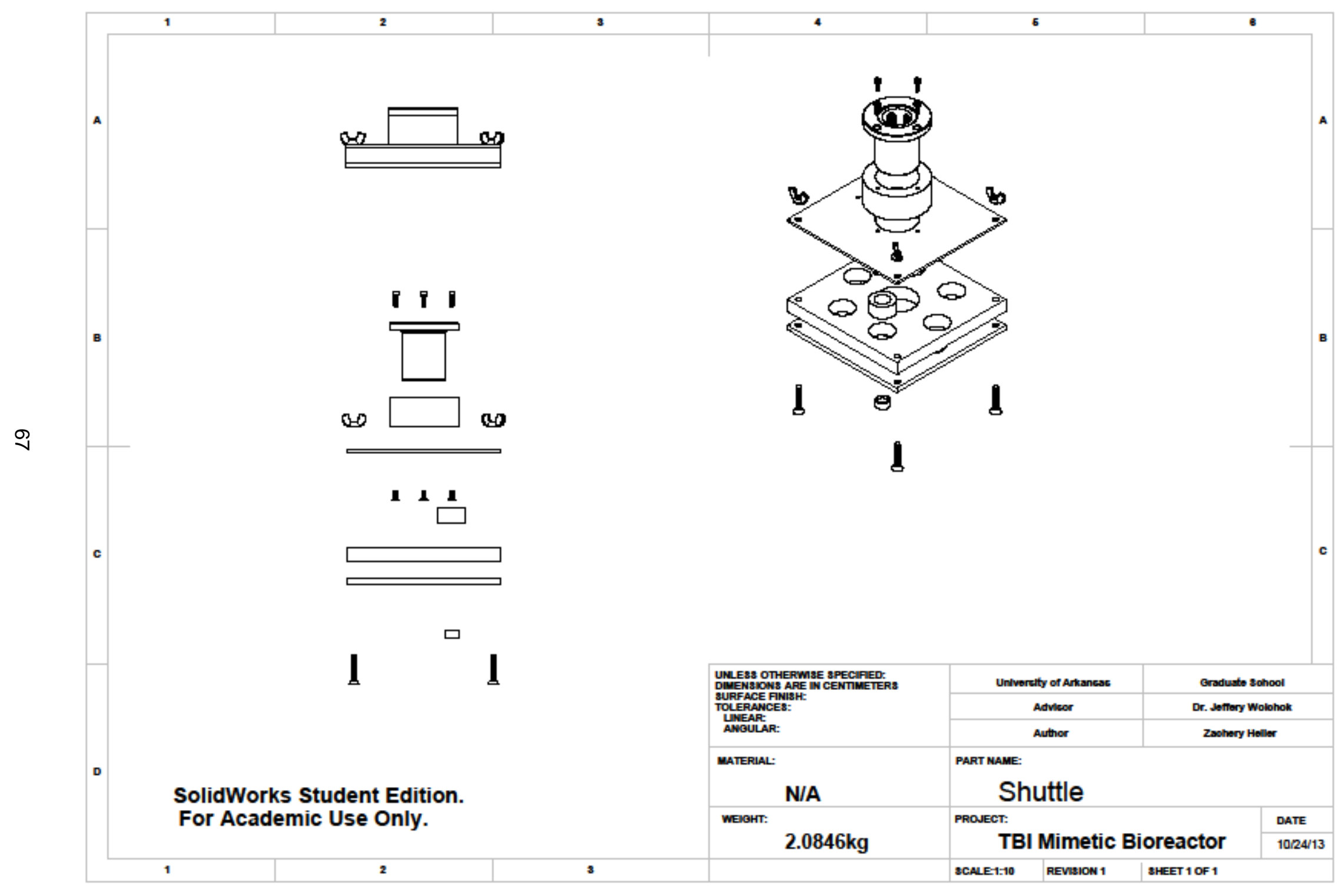


XII. APPENDIX C - Accelerometer Schematic

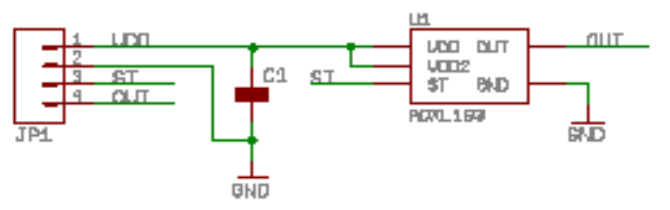

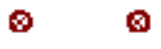

TITLE: ADXL193 Breakout-V11 Document Number:

FFE

Date: 2/1/2012 11:30:日B AH 